\title{
Integrated sedimentological and ichnological study of the Coniacian sedimentation in North Sudetic Basin, SW Poland
}

\author{
Stanisław LESZCZYŃSKI ${ }^{1, *}$ \\ 1 Jagiellonian University, Institute of Geological Sciences, Gronostajowa 3a, 30-063 Kraków, Poland
}

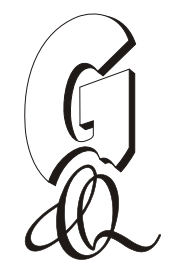

Leszczyński, S., 2018. Integrated sedimentological and ichnological study of the Coniacian sedimentation in North Sudetic Basin, SW Poland. Geological Quarterly, 62 (4): 767-816, doi: 10.7306/gq.1440

Associate editor: Tadeusz M. Peryt

Lithofacies and ichnological features of the Coniacian deposits of the upper part of erkowice Member and lowest part of the overlying Czerna Formation in southeastern part of the North Sudetic Synclinorium are described and their sedimentary palaeoenvironment is interpreted. The study confirms a shallow-marine to paralic/paludine palaeoenvironment. Sedimentation of the erkowice Member occurred in an upper shoreface environment dominated by waves, tidal currents and wave-generated alongshore currents, with an episodic encroachment of foreshore zone and shoal-water deltas. The interpretation is supported by a high-diversity assemblage of trace fossils with 21 ichnogenera, representing a stressed expression of the Skolithos Ichnofacies dominated by Ophiomorpha nodosa and a proximal expression of the Cruziana Ichnofacies with Thalassinoides and rare specimens of diverse other ichnotaxa. Sedimentation of the Czerna Formation commenced after a stasis, with at least a local hiatus caused by emergence, and proceeded in a laterally and vertically more varied environment, with transgressive coastal lagoons evolving into freshwater lakes and marshes and with a repetitive regressive intrusion of shoreface and shoal-water deltas. The emergence of the area is recorded by coal-bearing deposits with plant-root traces. Local occurrence of the Teredolites Ichnofacies in coal (peat) deposits above the base of the Czerna Formation indicates renewed marine flooding. Continuation of the latter is locally evidenced by a trace-fossil assemblage with 17 ichnogenera, representing proximal expression of the Cruziana Ichnofacies followed by distal expression of the Skolithos Ichnofacies in the overlying transgressive-regressive cyclothems. The palaeoenvironmental changes recorded by the sedimentary succession indicate bathymetric fluctuations and imply considerable shoreline shifts and palaeogeographic changes in the basin. These changes are interpreted as a combined signal of 2 nd- and 3rd-order eustatic cycles, modified and partly obliterated by the effects of intrabasinal tectonic forcing and by palaeogeographically controlled variation in sediment supply.

Key words: lithofacies, ichnofacies, trace fossils, shallow marine, paralic, North Sudetic Synclinorium.

\section{INTRODUCTION}

Cretaceous deposits (Cenomanian-Santonian), as regards their thickness and outcrop area, are a major stratigraphic component of the North Sudetic Synclinorium (NSS). Based on lithofacies and body fossils, the sedimentary environment of these deposits was recognized as shallow marine, ranging broadly from nearshore and shallow offshore to paralic (Andert, 1934; Scupin, 1936; Bassyouni, 1984; Milewicz, 1991a, b, 1997). Trace fossils abound, but - apart from a cursory interpretation of ichnofacies by Bassyouni (1984) - have thus far been little studied, although they are an important record of palaeoenvironmental conditions (see Taylor et al., 2003; Mcllroy, 2004; MacEachern et al., 2007a, b; Knaust and Bromley, 2012; Gingras

\footnotetext{
*E-mail: stan.leszczynski@uj.edu.pl

Received: March 29, 2018; accepted: May 30, 2018; first published online: December 12, 2018.
}

and MacEachern, 2012). The first detailed ichnological analysis has been only recently reported by Leszczyński (2010) from the sedimentological study of a single small section of the Coniacian succession, namely the lower part of the Czerna Formation of latest Coniacian age (Walaszczyk, 2008) in Rakowice Małe area. The ichnological data helped refine an earlier sedimentological interpretation of these deposits and indicated precisely the onset of a new episode of marine sedimentation after that of the underlying erkowice Member. This local study inspired the author to extend the integrated sedimentological and ichnological research to other areas and other parts of the Coniacian sedimentary succession. At the final stage of the work, it turned out that similar research was conducted simultaneousy in the erkowice Member by other researchers. Their study (Chrzastek and Wypych, 2016, 2018; Chrząstek et al., 2018) enriched data gained by the author, also in showing some controversial opinions concerning some trace fossils, ichnofacies and depositional environment.

The present paper reports on the results of the author's several years of sedimentological and ichnological research on the Coniacian deposits of the upper part of erkowice Member of 
the Rakowice Wielkie Formation (Milewicz, 1985) and lower part of the overlying Czerna Formation. The principal aims of this paper are to:

- describe macroscopic sedimentary features of both abiotic and biotic origin, including taxonomic classification of the latter and their distribution and association with the former in the Coniacian basin-fill succession;

- demonstrate the importance of ichnological data in the palaeoenvironmental reconstruction of complex shallow-marine successions with high lateral variability and frequent stratigraphic changes of basin conditions; and

- contribute to the general knowledge on Coniacian sedimentation and palaeogeography in the North Sudetic region.

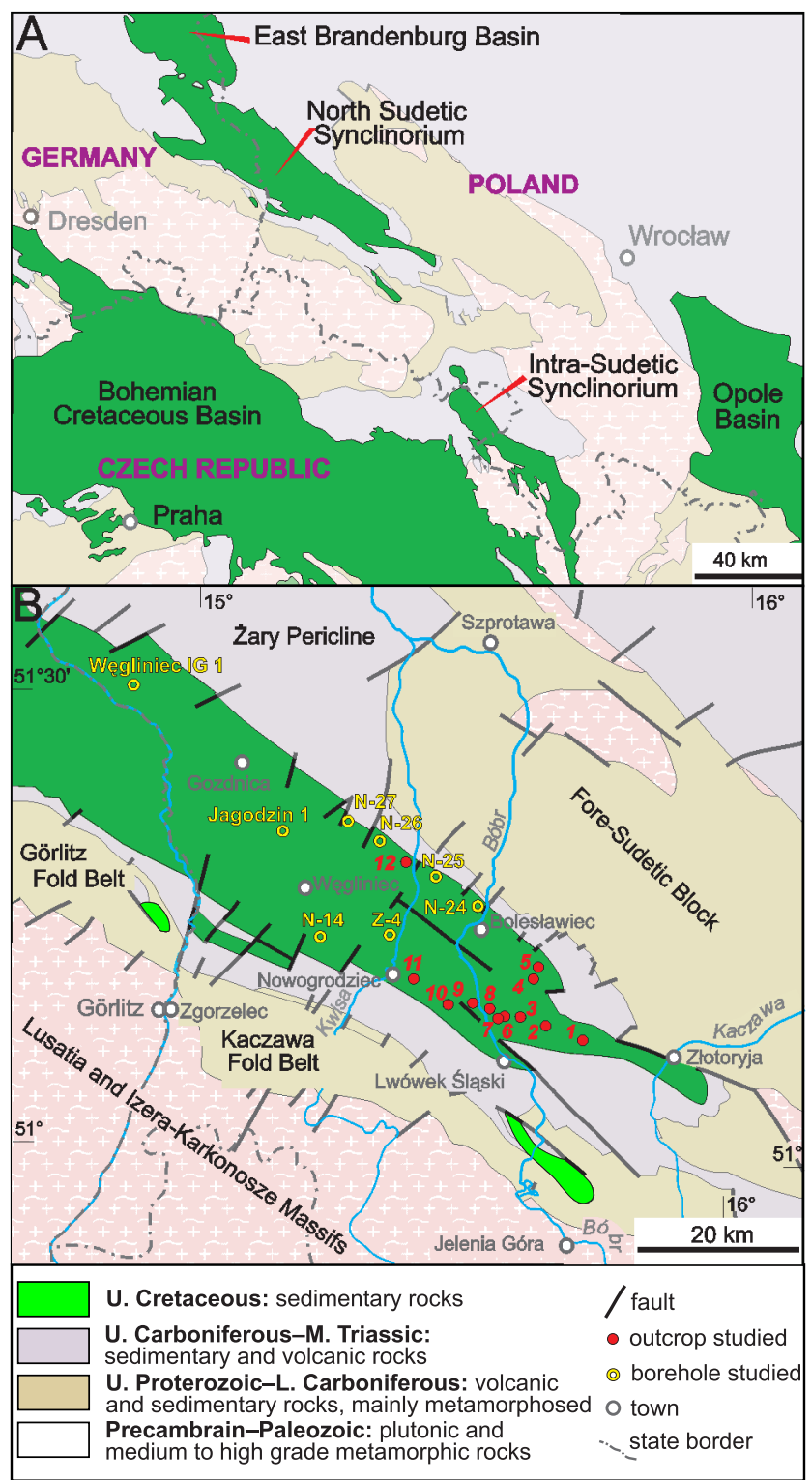

Fig. 1. Geographical and geological location of the study area

A - location of the study area in broader regional context (modified after Pożaryski et al., 1979); B - location of the outcrops and borehole profiles studied relative to the North Sudetic Synclinorium and its surroundings, without Cenozoic (compiled after Milewicz, 1997; Pożaryski et al., 1979). For closer information on the outcrop localities see Table 1

\section{REGIONAL GEOLOGICAL SETTING AND STRATIGRAPHY}

The study area is located in the southeastern part of the North Sudetic Synclinorium (NSS) (Fig. 1). The NSS represents one of several mid-Cretaceous basins that developed within and around the Bohemian Massif due to the stress field of the Alpine orogeny (Fig. 2). These basins formed by reactivation of older, mainly Variscan, shear zones dissecting the Bohemian Massif and its peripheries. The NSS formed as a southeastern extension of the East Brandenburg Basin (Musstow, 1968; Voigt et al., 2008), at the interface of Tethyan and Boreal realms (Fig. 2). To the north-east, the NSS is bordered by the elevated Fore-Sudetic Block and its north-western envelope known as the Żary Pericline. The Fore-Sudetic Block is devoid of Mesozoic rocks, whereas the Żary Pericline involves Permian to Middle Triassic deposits. To the southwest, the NSS is bordered by the elevated Görlitz Fold Belt and the Kaczawa Greenstone-and-Slate Fold Belt made of pre-Permian metamorphic rocks (Żelaźniewicz et al., 2011).

Cretaceous deposits constitute the youngest part of a discontinuous Upper Palaeozoic-Mesozoic sedimentary succession in the NSS. These Cenomanian to Santonian deposits include sandstones, marlstones, mudstones and claystones with subordinate intercalations of other rocks, mainly limestones (Milewicz, 1965, 1997). Outcrops and drilling cores show considerable lateral and vertical variation of these deposits, with sandstone bodies pinching out in marlstones, limestones and mudstones towards the north-west (Figs. 3-5). Lithostratigraphic interpretation of the sedimentary succession has evolved with time (see Milewicz, 1997). The early German geologists in the region referred to the thick sandstone units as Quadersandstein (Beyrich, 1849). The current lithostratigraphy was proposed by Milewicz (1985), who subdivided the succession into three roughly defined formations. The lower part of the succession, comprising the sandstone units with intervening marlstones and coeval marlstones and limestones, was distin-

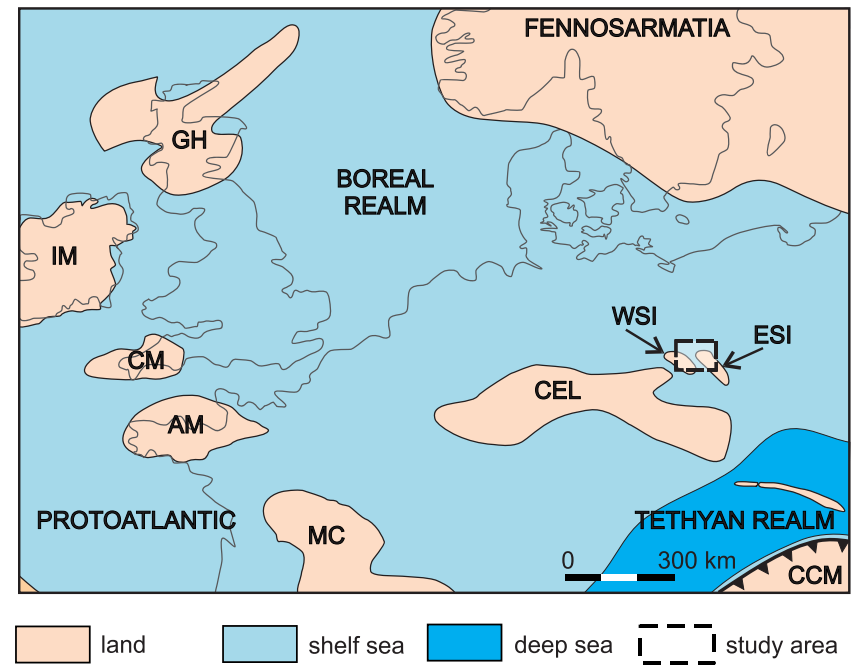

Fig. 2. Palaeogeographic location of the North Sudetic Cretaceous Basin (modified from Ziegler, 1990; Nádaskay and Uličný, 2014)

Land masses: AM - Armorican Massif, CCM - Central Carpathian Massif, CEL - Central European Land, CM - Cornubian Massif, ESI - East Sudetic Island, GH - Grampian High, IM - Irish Massif, MC Massif Central, WSI - West Sudetic Island 
List of outcrop localities (labelled 1-12) and their characteristics

\begin{tabular}{|c|c|c|c|c|}
\hline No. & Locality name & $\begin{array}{l}\text { Outcrop GPS } \\
\text { coordinates }\end{array}$ & Outcrop type & General features of exposed succession \\
\hline \multirow{4}{*}{1.} & \multirow{4}{*}{$\begin{array}{l}\text { Czaple } \\
\text { (four outcrops } \\
\text { labelled A-D) }\end{array}$} & $\begin{array}{l}\text { Outcrop A } \\
51^{\circ} 08^{\prime} 29^{\prime \prime} \mathrm{N} \\
15^{\circ} 45^{\prime} 36^{\prime \prime} \mathrm{E}\end{array}$ & $\begin{array}{c}\text { Active quarry at the top of Kopka Hill, } \\
\text { operated by company } \\
\text { Gruszecki Sp. z o.o. }\end{array}$ & \multirow{4}{*}{$\begin{array}{c}\text { Thick- and very thick-bedded sandstones of the } \\
\text { erkowice Member (Rakowice Wielkie Formation) in all } \\
\text { outcrops. Small local relics of the lowermost part of } \\
\text { Czerna Formation (variegated claystones, mudstones } \\
\text { and sandstones) in outcrop B }\end{array}$} \\
\hline & & $\begin{array}{l}\text { Outcrop B } \\
51^{\circ} 08^{\prime} 28^{\prime \prime} \mathrm{N} \\
15^{\circ} 45^{\prime} 40^{\prime \prime} \mathrm{E}\end{array}$ & $\begin{array}{c}\text { Active quarry at the top of Kopka Hill, } \\
\text { operated by company } \\
\text { Kamieniarz Sp. z o.0. }\end{array}$ & \\
\hline & & $\begin{array}{l}\text { Outcrop C } \\
51^{\circ} 08^{\prime} 32^{\prime \prime} \mathrm{N} \\
15^{\circ} 45^{\prime} 11^{\prime \prime} \mathrm{E} \\
\end{array}$ & $\begin{array}{l}\text { Abandoned quarry on southwestern } \\
\text { slope of Kopka Hill, owned by company } \\
\text { Kamieniarz Sp. z o.o. }\end{array}$ & \\
\hline & & $\begin{array}{l}\text { Outcrop D } \\
51^{\circ} 08^{\prime} 41^{\prime \prime} \mathrm{N} \\
15^{\circ} 44^{\prime} 52^{\prime \prime E}\end{array}$ & $\begin{array}{l}\text { Abandoned quarry at the western foot } \\
\text { of Kopka Hill, owned by company } \\
\text { Kamieniarz Sp. z o.o. }\end{array}$ & \\
\hline 2. & Zbylutów & $\begin{array}{l}51^{\circ} 08^{\prime} 57^{\prime \prime} \mathrm{N} \\
15^{\circ} 39^{\prime} 31^{\prime \prime E}\end{array}$ & $\begin{array}{c}\text { Active guarry Zbylutów IV, operated by } \\
\text { Kopalnia Piaskowca JAN }\end{array}$ & $\begin{array}{l}\text { Thick- and very thick-bedded sandstones of the } \\
\text { erkowice Member (Rakowice Wielkie Formation) }\end{array}$ \\
\hline 3. & Gaszów & $\begin{array}{l}51^{\circ} 09^{\prime} 32 " \mathrm{~N} \\
15^{\circ} 37^{\prime} 5 " \mathrm{E}\end{array}$ & $\begin{array}{l}\text { Long-abandoned quarry, } \\
\text { overgrown by vegetation }\end{array}$ & $\begin{array}{l}\text { Very thick- and thick-bedded sandstones of the } \\
\text { erkowice Member (Rakowice Wielkie Formation) }\end{array}$ \\
\hline 4. & eliszów & $\begin{array}{l}51^{\circ} 11^{\prime} 5 " \mathrm{~N} \\
15^{\circ} 38^{\prime} 54^{\prime \prime} \mathrm{E}\end{array}$ & $\begin{array}{l}\text { Recently re-activated quarry operated } \\
\text { by company Kamieniarz Sp. z o.0. }\end{array}$ & $\begin{array}{l}\text { Thick- and very thick-bedded sandstones of the } \\
\text { erkowice Member (Rakowice Wielkie Formation) }\end{array}$ \\
\hline 5. & Wartowice & $\begin{array}{l}51^{\circ} 12^{\prime} 45^{\prime \prime} \mathrm{N} \\
15^{\circ} 39^{\prime} 12^{\prime \prime} \mathrm{E}\end{array}$ & $\begin{array}{c}\text { Active quarry operated by company } \\
\text { Hofmann Natursteinwerke Polen, } \\
\text { GmbH }\end{array}$ & $\begin{array}{l}\text { Thick- and very thick-bedded sandstones with one or } \\
\text { two intercalations of mudstones and heterolithic depos- } \\
\text { its ( erkowice Member of Rakowice Wielkie Forma- } \\
\text { tion), overlain by grey and variegated mudstones with } \\
\text { thin coal and sandstones (Czerna Formation) }\end{array}$ \\
\hline 6. & Skała I & $\begin{array}{l}51^{\circ} 09^{\prime} 35^{\prime \prime} \mathrm{N} \\
15^{\circ} 34^{\prime} 57^{\prime \prime} \mathrm{E}\end{array}$ & $\begin{array}{c}\text { Active quarry operated by company } \\
\text { Hofmann Natursteinwerke Polen, } \\
\text { GmbH }\end{array}$ & $\begin{array}{l}\text { Very thick-bedded sandstones of the erkowice } \\
\text { Member (Rakowice Wielkie Formation) }\end{array}$ \\
\hline 7. & Skała II & $\begin{array}{l}51^{\circ} 09^{\prime} 30^{\prime \prime} \mathrm{N} \\
15^{\circ} 34^{\prime} 30^{\prime \prime} \mathrm{E}\end{array}$ & Rock tor "Medalion" & $\begin{array}{l}\text { Very thick-bedded sandstones of the erkowice } \\
\text { Member (Rakowice Wielkie Formation) }\end{array}$ \\
\hline 8. & erkowice & $\begin{array}{l}51^{\circ} 09^{\prime} 40^{\prime \prime} \mathrm{N} \\
15^{\circ} 34^{\prime} 26^{\prime \prime} \mathrm{E}\end{array}$ & $\begin{array}{l}\text { Active quarry operated by company } \\
\text { Kopalnie Piaskowca S.A. Bolesławiec }\end{array}$ & $\begin{array}{c}\text { Very thick-bedded sandstones of the erkowice } \\
\text { Member (Rakowice Wielkie Formation) }\end{array}$ \\
\hline 9. & $\begin{array}{l}\text { Rakowice Małe } \\
\text { (Rakowiczki) }\end{array}$ & $\begin{array}{l}51^{\circ} 9^{\prime} 57^{\prime \prime N} \\
15^{\circ} 32^{\prime} 33^{\prime \prime E}\end{array}$ & $\begin{array}{l}\text { Recently abandoned quarry owned by } \\
\text { Kopalnie Piaskowca S.A. Bolesławiec }\end{array}$ & $\begin{array}{c}\text { Very thick-bedded sandstones of the erkowice } \\
\text { Member (Rakowice Wielkie Formation) overlain } \\
\text { by variegated and grey mudstones with thin coal } \\
\text { seams, heterolithic deposits and thin to thick } \\
\text { sandstone beds (Czerna Formation) }\end{array}$ \\
\hline 10. & Kotliska & $\begin{array}{l}51^{\circ} 09^{\prime} 48 " \mathrm{~N} \\
15^{\circ} 30^{\prime} 51^{\prime \prime} \mathrm{E}\end{array}$ & $\begin{array}{c}\text { Long-abandoned quarry, overgrown } \\
\text { by vegetation }\end{array}$ & $\begin{array}{l}\text { Very thick-bedded sandstones of the erkowice } \\
\text { Member (Rakowice Wielkie Formation) }\end{array}$ \\
\hline 11. & Milików & $\begin{array}{l}51^{\circ} 11^{\prime} 13^{\prime \prime} \mathrm{N} \\
15^{\circ} 26^{\prime} 18^{\prime \prime} \mathrm{E}\end{array}$ & $\begin{array}{l}\text { An array of closely spaced, long- } \\
\text { abandoned and overgrown quarries }\end{array}$ & $\begin{array}{c}\text { Very thick-bedded sandstones of the erkowice } \\
\text { Member (Rakowice Wielkie Formation) }\end{array}$ \\
\hline 12. & Osiecznica & $\begin{array}{l}51^{\circ} 19^{\prime} 40^{\prime \prime} \mathrm{N} \\
15^{\circ} 23^{\prime} 45^{\prime \prime} \mathrm{E}\end{array}$ & $\begin{array}{c}\text { Active sand pit operated by Kopalnia i } \\
\text { Zakład Przeróbczy Piasków } \\
\text { Szklarskich Sp. z o.0. }\end{array}$ & $\begin{array}{l}\text { Indistinctly bedded, weakly cemented sandstones with } \\
\text { several thin mudstone intercalations in top part of } \\
\text { the erkowice Member (Rakowice Wielkie Formation), } \\
\text { overlain by dark grey mudstones with two packages } \\
\text { of heterolithic deposits and sandstone-dominated } \\
\text { top part (Czerna Formation) }\end{array}$ \\
\hline
\end{tabular}

For their map location, see Figure 1B

guished as the Rakowice Wielkie Formation (RWFm). The individual sandstone units within the RWFm were distinguished as separate members (Fig. 3). The present study focuses on the uppermost of these units, at the top of the RWFm in the southern to southeastern part of the NSS, which was named the erkowice Member (ZMb) by Milewicz (1985) and earlier called Oberer Quadersandstein (Drescher, 1863; Williger, 1882).

The $\mathrm{ZMb}$ is up to $\sim 100 \mathrm{~m}$ thick and composed of fine- to medium-grained and subordinately coarse-grained quartz arenitic sandstones of a cream-yellow to light-orange and greyish-white colour, weakly cemented to nearly soft (Milewicz, 1965, 1985, 1991a, 1997; Engel et al., 1978). Mudstone interbeds occur locally, particularly at the unit top. The sandstones are poorly bedded and range from medium to large-scale trough cross-stratified and tabular planar cross-stratified to massive, plane-parallel stratified and ripple cross-laminated, locally burrowed (Milewicz, 1965, 1997; Bassyouni, 1984). Escape and dwelling traces, considered to represent an undefined Monocraterion Ichnofacies or the Cruziana Ichnofacies, were mentioned by Bassyouni (1984). Body fossils are rare, represented by bivalves (including inoceramids), gastropods, ammonites and echinoids (Milewicz, 1965).

The lower boundary of the ZMb is relatively sharp, with the underlying dark grey mudstones overlain by massive, mainly fine-grained, quartzose sandstones. However, the boundary in some areas (e.g., in borehole N24) is less distinct and remains 


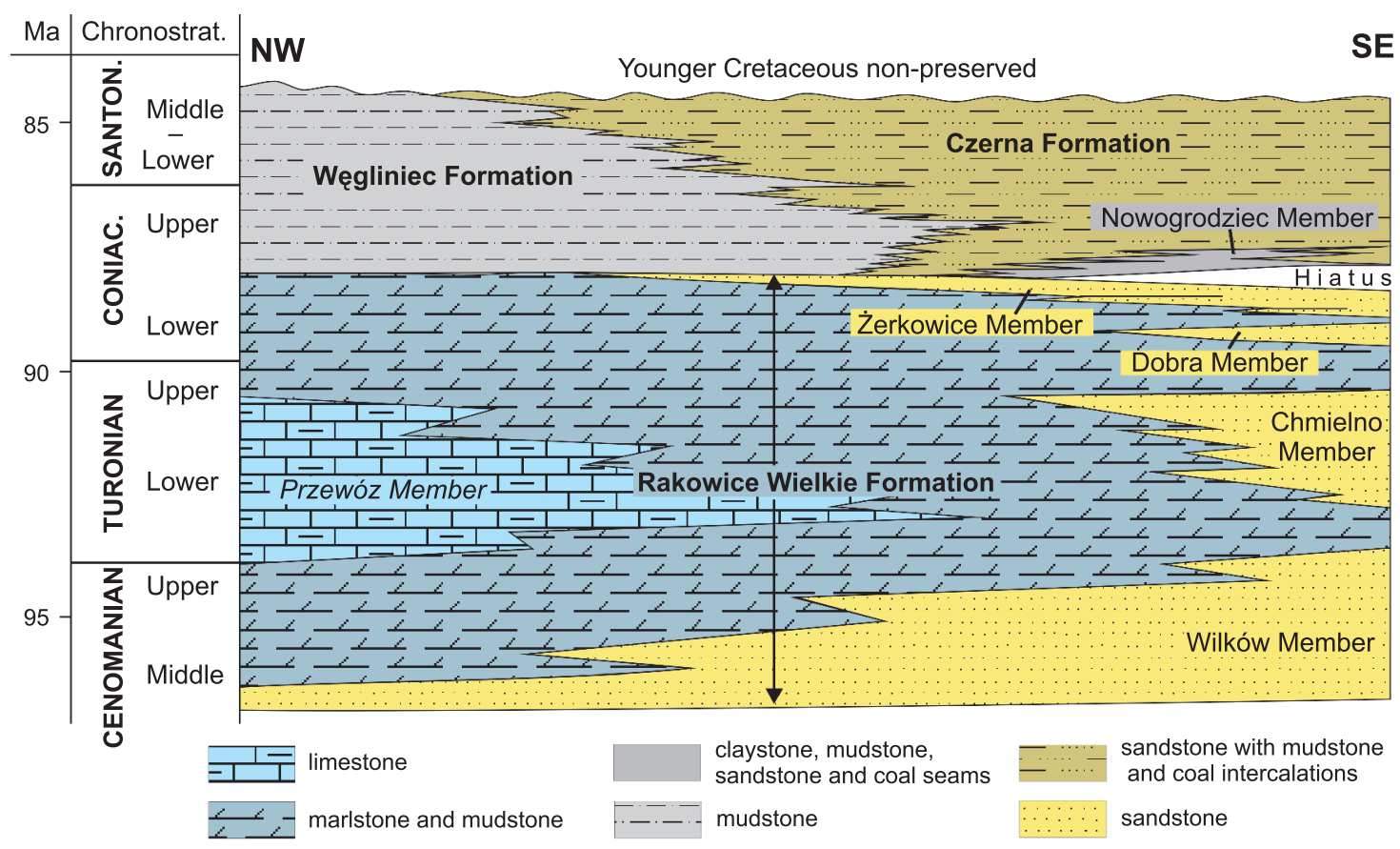

Fig. 3. Stratigraphy of the Cretaceous deposits in an axial cross-section of the North Sudetic Basin; lithostratigraphy after Milewicz (1997); chronostratigraphy after Walaszczyk (2008); numerical age from Cohen et al. (2013)

controversial, as the sandstone lithofacies there extend downwards to the lower Coniacian and upper Turonian (Bossowski, 1991b). The upper boundary is sharp in most areas (Fig. 6), marked by the contact of sandstones with the overlying package of dominantly claystones and mudstones. This contact also constitutes the upper boundary of the RWFm.

The deposits above the $\mathrm{ZMb}$ are loosely subdivided into two coeval formations, referred to as the Węgliniec Formation (WFm) and Czerna Formation (CFm) (Fig. 3; Milewicz, 1985). The WFm consists almost exclusively of mudstones, whereas the CFm, which occupies the southeastern part of the NSS, comprises sandstones intercalated with claystones and thin coal beds (Milewicz, 1965). The lowermost part of the CFm (2-50 $\mathrm{m}$ thick) - comprising limnic claystones and resting directly on the ZMb - was distinguished by Milewicz (1985) as the Nowogrodziec Member (NMb).

In the outcrop section at Rakowice Małe, Leszczyński (2010) has recognized horizons with plant-root casts and other bioturbation structures, including 14 ichnogenera, in the NMb and the directly overlying part ( $\sim 9 \mathrm{~m})$ of the CFm. Animal trace fossils were interpreted as representing the Skolithos, Teredolites and Cruziana ichnofacies. The CFm contains also horizons with brackish bivalves and gastropods (Drescher, 1863; Milewicz, 1965, 1997).

The first biostratigraphic dating of the Cretaceous of the NSS was given by Beyrich (1855), who interpreted these deposits as Cenomanian-Senonian. Milewicz (1958) confirmed a Santonian age of the upper part of the succession, comprising the CFm and WFm. Krutzsch (1966) specified the age of this part of succession as early Santonian. Milewicz $(1956,1979)$ suggested a late Coniacian (late Emscherian) hiatus in the southeastern part of the NSS, where - in the subsequent terminology of Milewicz (1985) - the ZMb is overlain directly by the CFm.
A somewhat different view of the succession's chronostratigraphy was given by Walaszczyk (2008), with the boundary between the RWFm and the overlying formations asigned to the middle/late Coniacian transition and with the Coniacian/Santonian boundary placed in the middle of the CFm and coeval WFm (Fig. 3). The hiatus was shown as corresponding to the middle Coniacian. This modified chronostratigraphy is followed in the present paper, although it should be noted that the Walaszczyk (2008) stratigraphic scheme is mistaken in showing a non-existent extension of marlstones over the entire ZMb (cf. Leszczyński, 2010).

\section{PREVIOUS VIEWS ON PALAEOENVIRONMENT AND PALAEOGEOGRAPHY}

The Cretaceous deposits of the NSS have been known since the 19th century to be of shallow marine to paralic origin, based on lithofacies and body fossils. A marine origin was supported by Milewicz (1985) for the deposits he distinguished as the RWFm and the WFm (Fig. 3). Milewicz (1991b) interpreted the sandstone units of the RWFm, including the ZMb, as deposited in a shoreface to foreshore environment, whereas an offshore origin was suggested for the units dominated by marlstones, limestones and mudstones. Bassyouni (1984) inferred strong influence of tides on the sedimentation of sandstones. Lacustrine and swamp deposits were recognized in the NMb, whereas brackish and deltaic to alluvial environment was suggested for the overlying part of the CFm (Milewicz, 1965, 1991b, 1997). Leszczyński (2010) elaborated further on the depositional environment of the NMb on the basis of lithofacies and ichnological data from the outcrop section in Rakowice Małe. 
$\mathrm{N}-14$

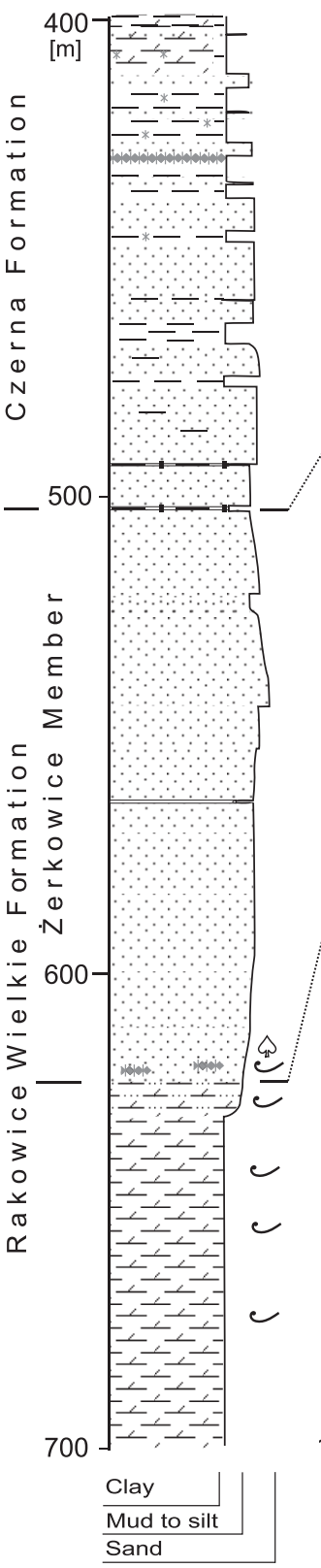

Z-4

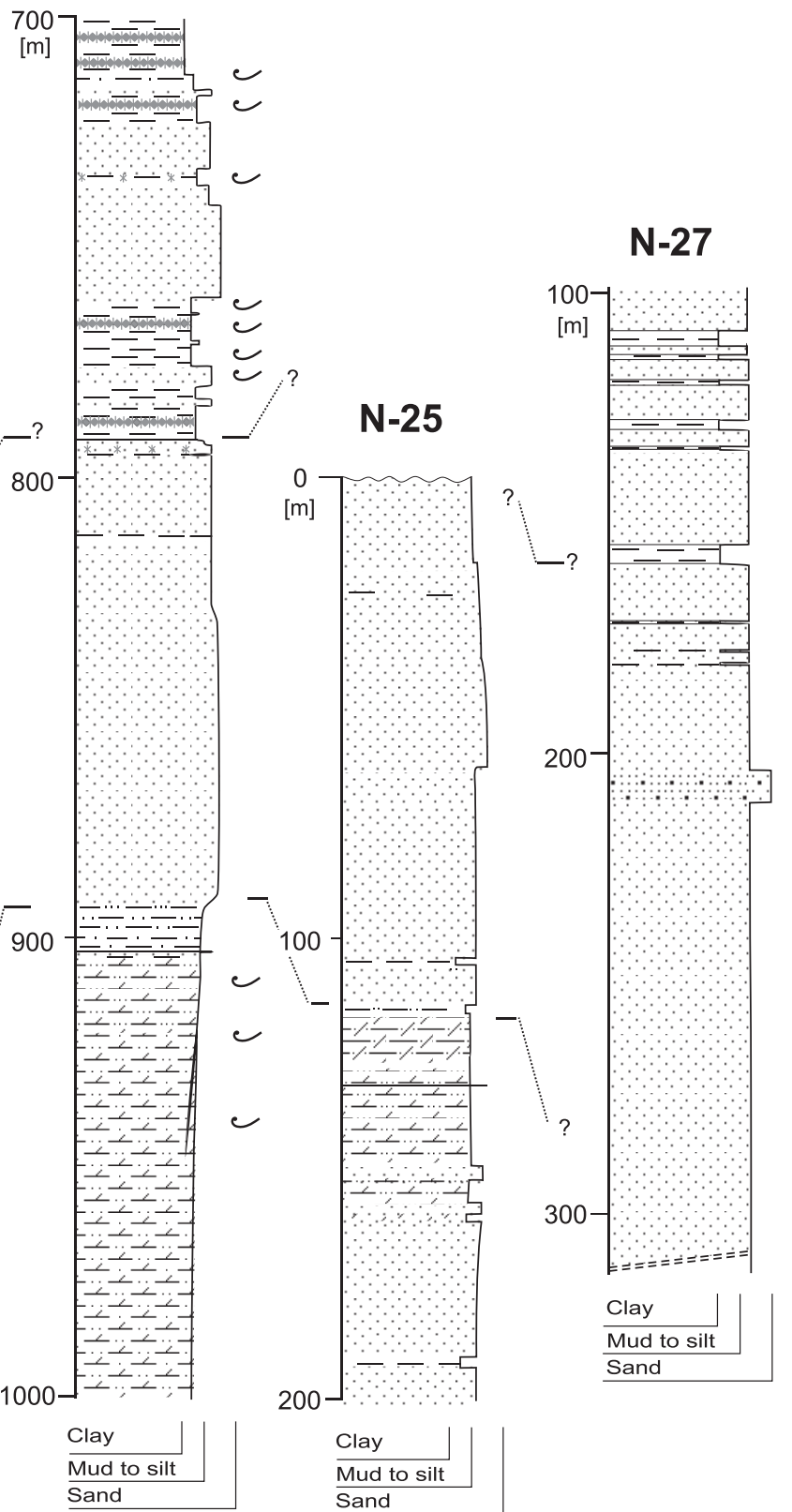

LEGEND

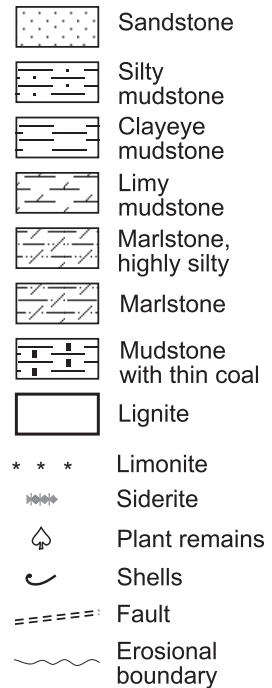

Fig. 4. Example borehole logs of the Żerkowice Member and the overlying lower part of Czerna Formation

Log N-14 is based on Bossowski et al. (1976), Bossowski (1991a); log Z-4 is based on Kochanowska (1988) and the present author's own logging of the borehole core; log N-25 is based on Dyja (1978) and Bossowski et al. (1978);

$\log \mathrm{N}-27$ is based on Bossowski et al. (1977); see borehole code above the log and location in Figure 1B

Palaeontological data collected over nearly two centuries have indicated that the Cretaceous sedimentation of the North Sudetic Basin commenced with the worldwide Cenomanian transgression and proceeded until at least the middle Santonian (see Milewicz, 1997; Walaszczyk, 2008). Partsch (1896) suggested a connection of the North Sudetic Basin with the Bohemian Basin during the Late Cretaceous. Scupin (1910) argued that the North Sudetic Cretaceous was deposited in a south-east-north-west trending basin, the North Sudetic Basin, bordered by elevated island areas to the north-east and southwest (Fig. 2). He called these elevated areas the Ostsudetische
Landmasse (East Sudetic Landmass) and Riesengebirgsinsel (West Sudetic Island), respectively. Scupin also advocated a connection of the North Sudetic Basin with the Central Sudetic (Intrasudetic) Basin since the late Cenomanian. Later on, Scupin (1912-1913) recognized a transition of sandy deposits into marlstones towards the north-west, which indicated the direction of basin deepening. The notion of two Cretaceous landmasses in the Sudetes was supported by Andert (1934), although the land area to the south-west of the North Sudetic Basin was suggested to have comprised two islands, referred to as the Lausitz Insel and Riesengebirges Insel. The latter was 


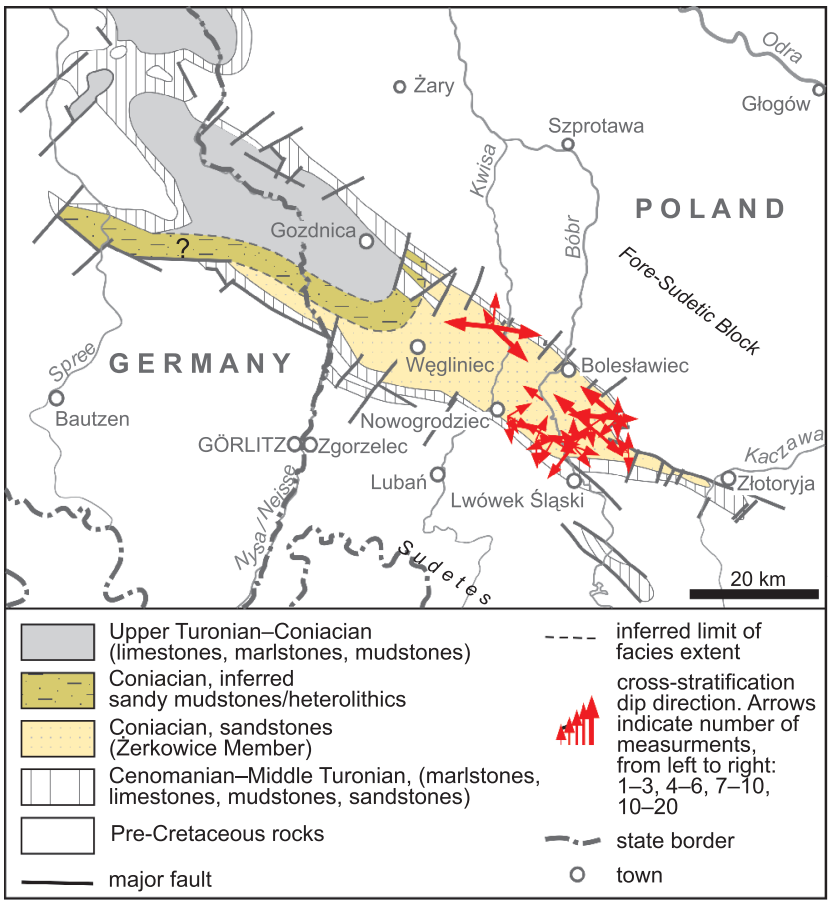

Fig. 5. Distribution of main lithologies coeval with Żerkowice Member in the North Sudetic Synclinorium and palaeocurrent directions measured in sandstones envisaged as a small island to the south-east of the basin, corresponding to a small part of Scupin's (1910) Ostsudetische Landmasse. Andert (1934) postulated further a periodical complete drowning of the islands.

Apart from its details, the palaeogeographic framework postulated by Scupin (1910) remains valid. The stratigraphic architecture of the deposits has been studied since the early 20th century to recognize regional palaeogeographic changes, which brought modifications to the basin stratigraphy and new data on the development of sedimentary environment. Particularly significant was the recognition of the late Coniacian hiatus in the southeastern part of the basin (Milewicz, 1956), as well as new data on stratigraphy and lithofacies distribution in the northwestern part of the basin (Milewicz, 1965, 1966, 1973; Milewicz et al., 1968). Milewicz (1997) envisaged a northwestward retreat of the sea from a large part of the ba$\sin$ at the final stages of the ZMb sedimentation, with the emerged area eroded in the late Coniacian (or late mid-Coniacian according to Walaszczyk, 2008). The new, subsequent episode of deposition there (WFm and CFm; Fig. 3) was recognized to have commenced in a lacustrine to paludal environment followed by deltaic sedimentation. In contrast, marine sedimentation proceeded throughout the Coniacian until the middle Santonian in the northwestern part of the basin, without a corresponding late mid-Coniacian hiatus (Milewicz, 1958, 1965, 1970, 1997, 2006; Walaszczyk, 2008).

Taken together, the previous studies indicate that the stratigraphic architecture of the North Sudetic Cretaceous reflects an interplay between the sediment supply and accommodation space controlled by both eustatic and tectonic forcing.
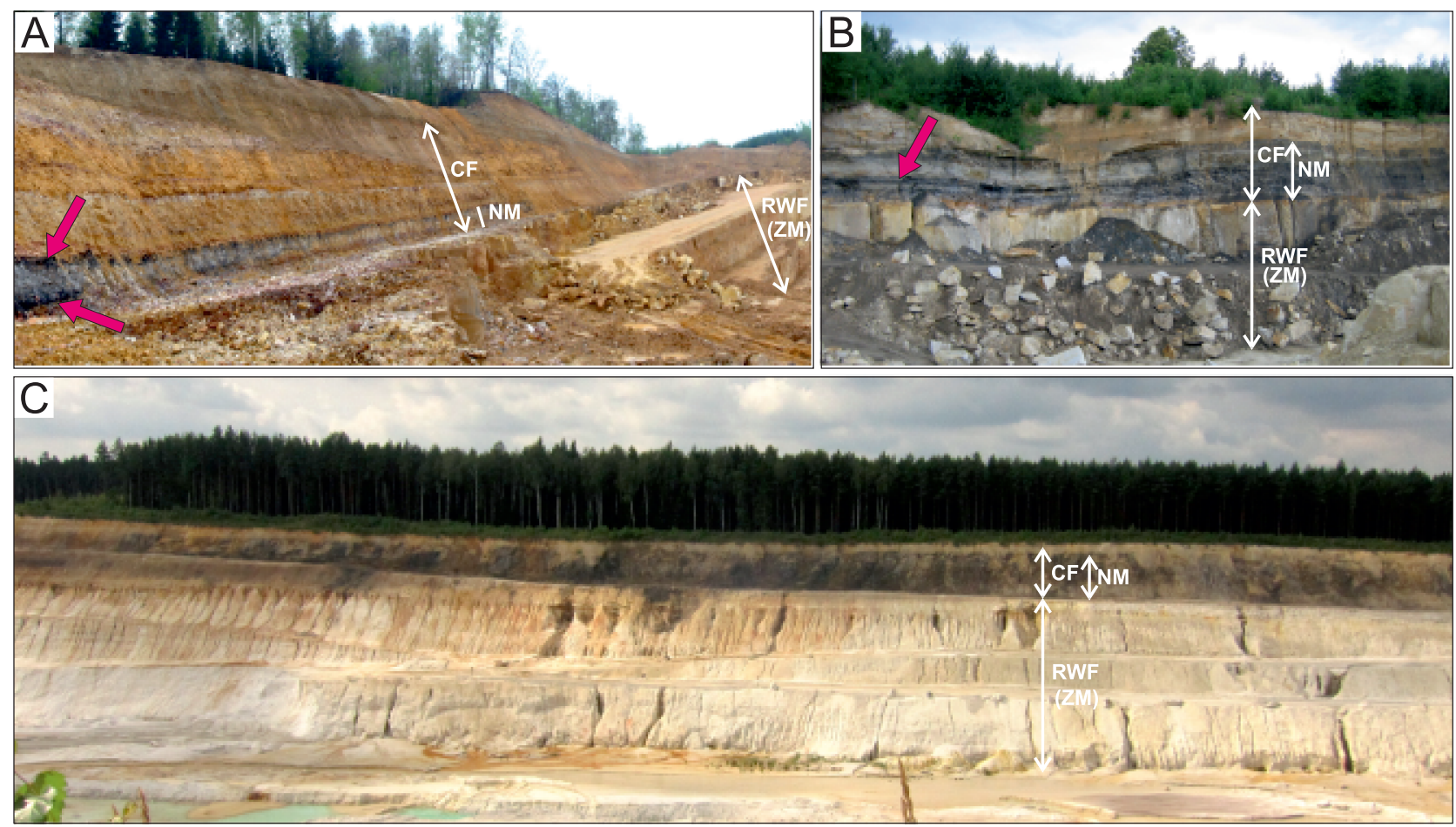

Fig. 6. Outcrops of the upper part of Żerkowice Member and the lower part of the overlying Czerna Formation

A - locality 5 (Wartowice), photography 22 August 2011; B - locality 8 (Żerkowice), photography 5 July 2007; C - locality 12 (Osiecznica), photography 9 August 2012. CF - Czerna Formation, NM - Nowogrodziec Member, RWF - Rakowice Wielkie Formation, ZM - Żerkowice Member.The red arrows point to coal beds; outcrop localities as in Table 1 and their location in Figure 1B 
According to Milewicz (1997), the Coniacian-Santonian of the NSS constitutes the top part of a regressive phase of a transgressive-regressive cycle (T-R cycle) and basal part of a transgressive phase of the next T-R cycle. Leszczyński (2010) has interpreted the ZMb as a regressive systems tract formed at the culmination of the regressive phase and separated by a hiatus from the overlying deposits (lowest CFm with NMb; Fig. 3) of a third-order transgressive systems tract. Earlier, Walaszczyk (2008) suggested that the CFm and coeval WFm represent the final, complex regressive phase of minor transgressive-regressive cycles.

\section{MATERIAL AND METHODS}

The present study examined outcrop sections in 12 active and abandoned quarries and a rock tor (Fig. 1 and Table 1), in addition to pre-existing 31 borehole profiles. The outcrops are located in the southeastern NSS and mainly in the southeastern part of the area occupied by the ZMb. Outcrops 5, 9 and 12 (Fig. 1) show both the upper part of the ZMb and the lower part of the CFm (Fig. 6), with the thickest portion of the ZMb ( 90 m) exposed in the last-mentioned exposure and with the exposed thickness of the CFm reaching 12-19 $\mathrm{m}$. The other outcrops show only the upper part of the ZMb, up to $\sim 15 \mathrm{~m}$ thick, with up to a few metres of the CFm exposed in outcrops $1 \mathrm{~B}$ and 4 (Fig. 1). Data on the whole ZMb succession were collected from borehole documentations. Except for the boreholes Z-4 and Węgliniec IG 1, from which poor-quality cores - crushed and in part as jumbled rock fragments - were available, the information was limited to the rock type, grain size, colour, consolidation degree and the well footage. Detailed data on sediment features and trace fossils were collected in outcrop sections.

The outcrop sections were analysed and logged bed-by-bed as far as possible. Bedding attitude and palaeocurrent directions (mainly from cross-stratification) were measured. Sediment features were analysed macroscopically both in the outcrop wall and loose rock blocks, particularly those extracted by mining works. Characteristic features were photographed. Some hardly accessible parts of outcrops were analysed remotely with the help of binoculars. The descriptive sedimentological terminology is according to Harms et al. $(1975,1982)$ and Collinson et al. (2006).

Sediment biotic features resulting from the activity of organisms are herein referred to broadly as bioturbation structures, irrespective of their form and size. Bioturbation structures showing morphologically the recurrent activity of organisms modifying the substrate are called trace fossils (Bertling et al., 2006) or ichnofossils. The description of trace fossils included their macroscopic form, abundance, as well as the type of encasing sediment and their stratinomic position. The interpretation of trace fossils included their taxonomic affiliation and ethological character. A large part of the trace-fossil data, particularly from bedding surfaces, was collected in loose rock blocks detached from the outcrop walls by mining activity. The exact original location of such blocks in the outcrop section was usually unclear, as was also the nature of the bedding surface (whether bed base or top) in some cases. Trace fossils were extensively photographed in the field, but specimens were not collected, except for a few of the most representative ones.

The taxonomic identity of trace fossils was determined for the better-preserved and more evident ones. Poorly preserved trace fossils were classified solely at the ichnogenus level or in open nomenclature (see Bengtson, 1988; Bertling et al., 2006). Each ichnotaxon is herein described with respect to its basic characteristics, stratigraphic location and literature references. All ichnotaxa, except for the few less distinctive ones, and already documented from the CFm by Leszczyński (2010), are illustrated with photographs ordered alphabetically according to ichnogenera and supplemented with description of basic features and lithostratigraphic provenance.

The degree of sediment bioturbation was determined according to the bioturbation index $(\mathrm{BI})$ scale of Taylor and Goldring (1993): $B I=0$, lack of bioturbation; $B I=1$, sparse bioturbation; $\mathrm{BI}=2$, low bioturbation; $\mathrm{BI}=3$, moderate bioturbation; $\mathrm{BI}=4$, high bioturbation (abiotic sediment structure indistinct due to biotic reworking); $\mathrm{BI}=5$, intense bioturbation (abiotic sediment structure completely disturbed); and $\mathrm{BI}=6$, complete bioturbation. The degree of bioturbation due solely to Ophiomorpha was described by the Ophiomorpha ichnofabric index (Oii) proposed by Anderson and Droser (1998). Photographs from active quarries include the date when the picture was taken specified in their captions.

\section{RESULTS}

\section{LITHOSTRATIGRAPHIC SEDIMENTARY CHARACTERISTICS}

The $\mathrm{ZMb}$ in all outcrops is dominated by fine- to medium-grained quartz arenites, weakly cemented to nearly soft. The hardest sandstone variety, still only moderately cemented, occurs in the southeastern part of the NSS, where it is quarried as a high quality building stone. Towards the north-west, the sandstone unit is practically soft and mined as a high-quality silica sand near the village of Osiecznica. Thin mudstone and claystone intercalations occur locally, particularly in the top part of the sandstone succession (Fig. 7). Most exposed sections show sandstone beds one to several metres thick, massive-looking to faintly cross-stratified or plane-parallel stratified (Figs. 8 and 9A, B). Wedge-shaped sets of planar cross-strata occur locally, particularly in the uppermost part of the succession. Beds are bounded by more or less distinct surfaces, erosional to depositional, marking sharp lithofacies change, particularly in occurrence of thin clayey or muddy sand intercalations. The less distinct surfaces are highly irregular in shape, variably oriented relative to their distinct counterparts, and extend laterally for several metres as the outline of some lithofacies bodies (Fig. 9A, B). Washouts and gutter casts of various size occur locally. Although most sandstones look massive, their close inspection suggests that at least some of such beds may in reality be partly or fully stratified, as the sand is well-sorted and its lamination generally faint to indistinct.

The sandstones locally show bioturbation structures. The highest concentration of burrows occurs in the upper part of beds, down to $\sim 1.5 \mathrm{~m}$ below their top bounding surfaces. This is particularly characteristic of beds overlain by mudstone or bounded by a sharp extensive surface. The topmost bed part, 10 to $15 \mathrm{~cm}$ thick, is in some cases totally burrowed $(\mathrm{BI}=5$ or 6 Figs. 7 and 10), with the bioturbation intensity decreasing downwards. Wood fragments of different size, aggregated imprints of large fishbones(?), shell pavements, horizons rich in shell debris recorded as imprints and casts, as well as mudstone clasts, occur locally (Fig. 11), mainly at localities 5, 6 and 8 (Fig. 1) Some large wood fragments are recorded as clusters of Teredolites, the casts of wood borings (Fig. 12). At localities 4 and 5 , some crowded accumulations of the sea snail Nerinea were found, recorded as casts and imprints. 
A

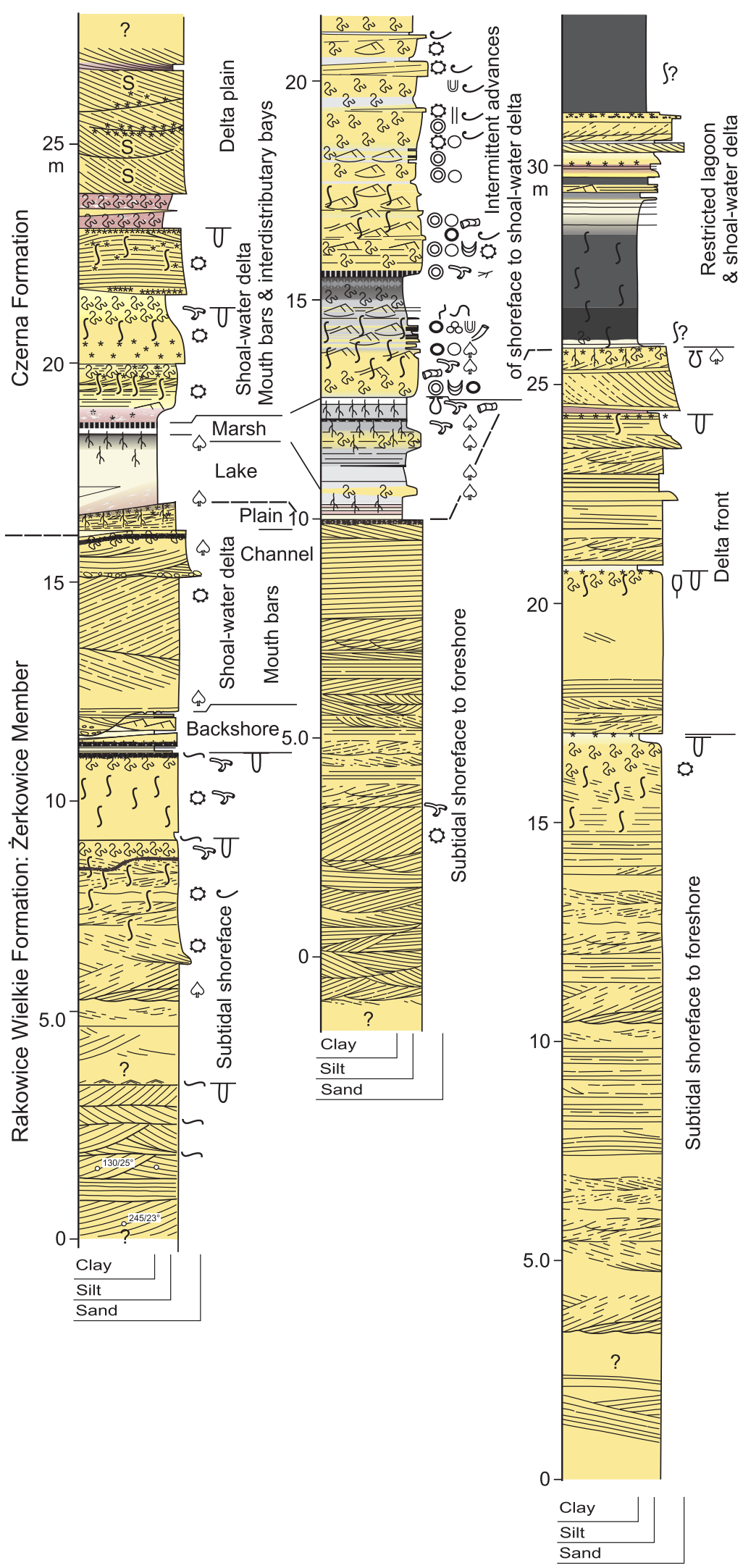

B C

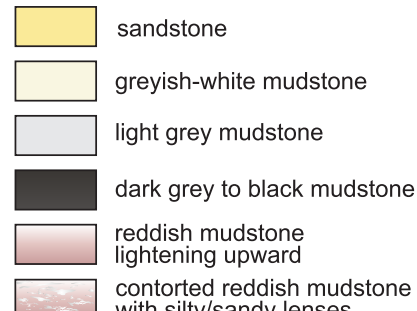

with silty/sandy lenses

|||||||| ||| coaly mudstone

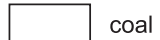

trough cross stratification

tabular cross stratification

$\overline{\overline{\overline{\overline{\bar{E}}}}}$ parallel stratification

a asymmetrical ripple

- symmetrical ripple

Ienticular bedding

=n wavy bedding

-.o. pebble-sized clasts

? features unknown

$S \quad$ features inferred

* Fe oxide/hydroxide-rich spot

* * * Fe oxide/hydroxide-rich horizon

ferricrete

siderite layer

4 plant debris

bioclasts (mainly shells)

$\int$ 즌 은 $\mathrm{Bl}=1-2$

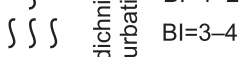

2 $\quad$ 드음 $\mathrm{Bl}=5-6$

$\sim$ epichnial bioturbation low to high

(U) Arenicolites \& A. - like burrows

(2) Asterosoma

7 Chondrites

D Cylindrichnus

ก Helminthopsis

O. Ophiomorpha

- Palaeophycus

- Planolites

O Rosselia

8 Schaubcylindrichnus

II Skolithos

Dos Taenidium

y Teichichnus

$\checkmark$ Teredolites

ऽ Thalassinoides

$r$ undetermined vermicular traces

h plant roots

$\int ? \quad$ undetermined trace fossils

U key stratal surface

boundary between Żerkowice Member (Rakowice Wielkie Formation) and Czerna Formation

Fig. 7. Compiled sedimentological logs of the most complete outcrop sections of the uppermost part of the Żerkowice Member and lowest part of the Czerna Formation

A - locality 5 (Wartowice), central part of quarry in August 2012; B - locality 9 (Rakowice Małe); C - locality 12 (Osiecznica) $\sim 1.3 \mathrm{~km}$ to NW from the starting point of the latter quarry; outcrop localities as in Table 1 and their map location in Figure $1 \mathrm{~B}$ 

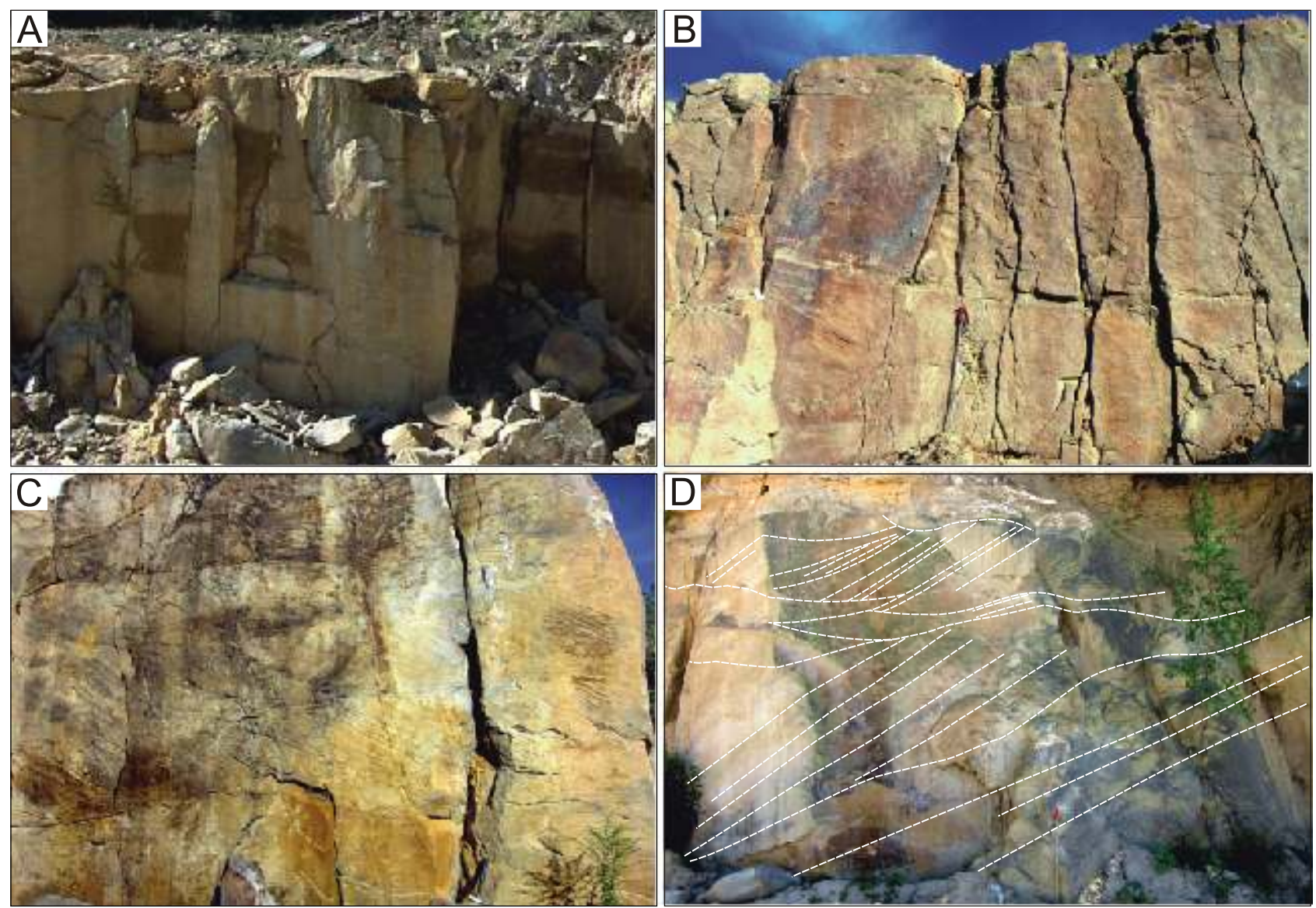

Fig. 8. Sandstone lithofacies of the erkowice Member

A - planar parallel stratified sandstone, locality 9 (Rakowice Małe), photography May 2007; B - massive and parallel stratified sandstone, locality $1 \mathrm{~A}$ (Czaple); photography September 2010, the walking stick (scale) is $1 \mathrm{~m}$ long; C - unidirectionally planar cross-stratified sandstone, locality 6 (Skała I), photography August 2013, the hammer (scale) is $35 \mathrm{~cm}$ long; D - large-scale cross-stratified and massive sandstones, locality 1D (Czaple), photography July 2015, the walking stick (scale) is $1 \mathrm{~m}$ long; outcrop localities as in Table 1 and their map location in Figure 1B

Cross-stratification sets are mainly $20-30 \mathrm{~cm}$ thick, but reaching a few metres and with strata poorly visible in well-sorted, clean quartzose sand. In outcrops 1C and 1D and in the northern part of quarry at locality 5 (Table 1), the ZMb top part, 3-4 $\mathrm{m}$ thick, is dominated by wedge-shaped planar and trough cross-stratification. At the latter locality, this cross-stratified unit is underlain by a mudstone-dominated heterolithic package, up to $60 \mathrm{~cm}$ thick, which in turn is underlain by a sandstone with a completely bioturbated top and rich Ophiomorpha below (Fig. 10A, C). Foreset strata dip in various directions, often parallel to the NSS axis (Fig. 5) and locally as bidirectional "herringbone" cross-stratification (Fig. 9A). The thickest cross-strata sets, up to several metres, occur in southeastern termination of the NSS. Set boundaries are locally covered with symmetrical or asymmetrical ripples (Fig. 9C-E) and show various burrows. Ripple marks are most common in the deepest parts of scoop-shaped surfaces bounding trough cross-strata sets. Planar parallel stratification is subordinate, in some cases inclined (Fig. 8A), and present mainly in the top part of the sandstone succession as sets up to several decimetres thick. Some of the stratified sandstone beds show soft-sediment deformation (Fig. 9D). The stratified sandstones are generally poor in bioturbation structures, although there are cases of strong bioturbation, with only remnants of stratification preserved.
Mudstones and claystones are minor components of the ZMb succession, present as thin layers between some of the thick sandstone beds or as thin heterolithic packages of interlayed mudstone, claystone and sandstone. These argillaceous deposits are well-compacted but soft, have a greyish white, light brown or pinkish-red colour and show parallel lamination or subtle mottling. These latter features are marked by the distribution of silt or fine sand and by colour differences.

The top part of the ZMb consists either of a weathered sandstone covered by Quaternary deposits or of a sandstone heavily stained with Fe-oxide/hydroxide and covered by the basal package of CFm deposits dominated by claystone and mudstone (Figs. 4 and 7). The boundary is characterized by orange, brown and crimson colours and the occurrence of a ferricrust. Ferruginization is most intense at locality 5 and nearly absent at locality 12 (Fig. 1).

At localities 1B, 5 and 12 (Fig. 1), the top part of the ZMb is a fine-grained sandstone unit, up to $5 \mathrm{~m}$ thick, with local admixtures of coarse sand grains and granules. Its base at localities 5 and 12 is erosional, underlain by a mudstone-dominated heterolithic package up to $60 \mathrm{~cm}$ thick, and the sandstone at locality 5 splits southwards into three beds separated by thin mudstone layers. The sandstone unit at all three localities shows wedges of planar cross-stratification, with strata sets up 

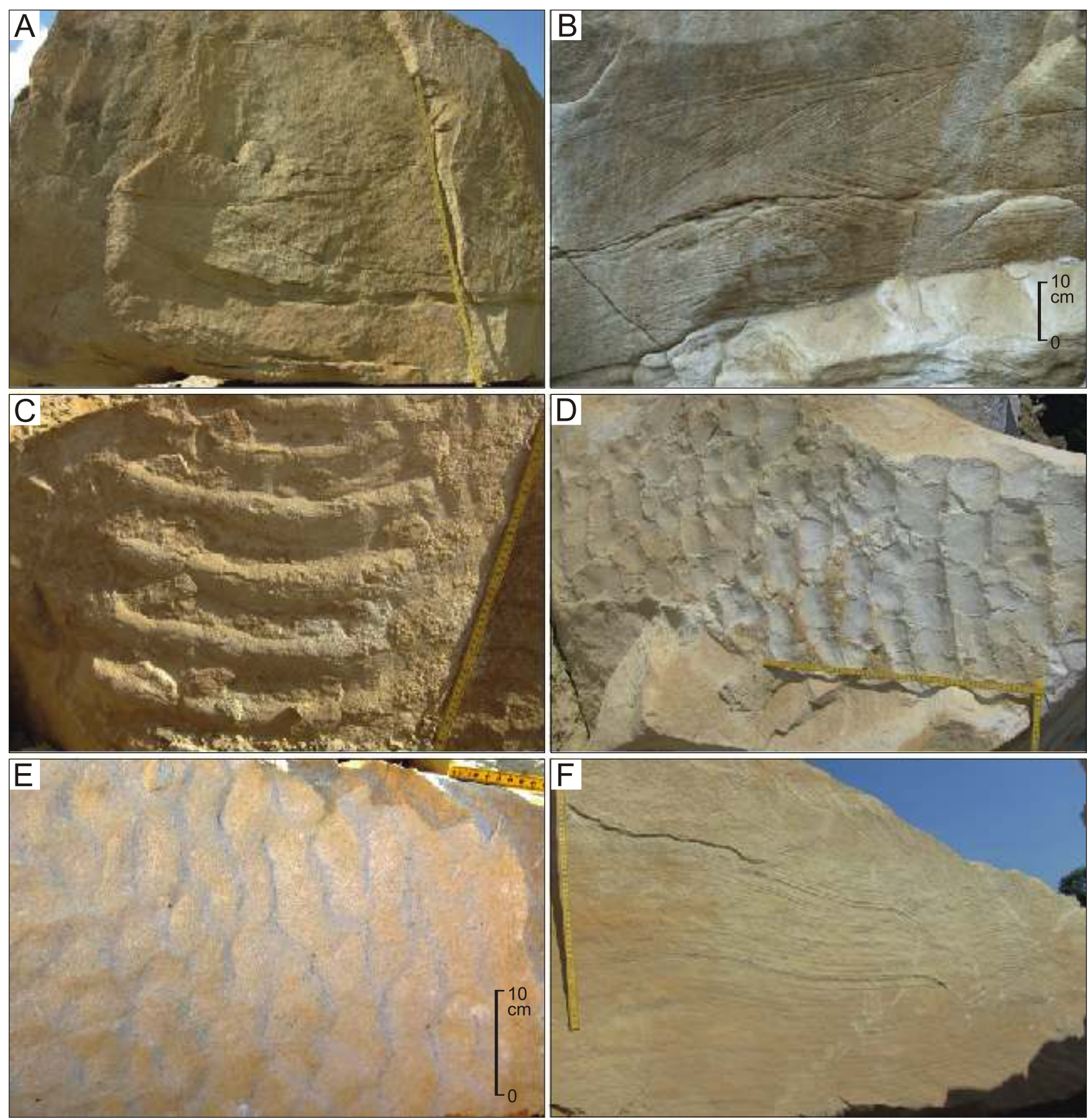

Fig. 9. Sandstone lithofacies of the erkowice Member

A - sandstone with a roughly unidirectional trough cross-stratification, locality 6 (Skała I), photography 23 August 2011; B - sandstones with planar parallel stratification and cross-stratification, including bidirectional (herringbone) cross-strata sets, locality 4 ( eliszów), photography October 2010; C - sandstone with vortex wave-ripple cross-lamination draping the scoop-shaped base of trough cross-strata set (seen from below), locality 6 (Skała I), photography 23 August 2011; D - rolling-grain wave-ripple lamination covering the top of sandstone bed, with a polygonal pattern of veins resembling desiccation crack-fills, same locality and date; $\mathbf{E}-$ combined-flow ripple cross-lamination at sandstone bed top, same locality and date; $\mathbf{F}$ - sandstone with planar parallel and cross-stratification, slightly soft-deformed, same locality and date; outcrop localities as in Table 1 and their map location in Figure 1B

to $2 \mathrm{~m}$ thick, and lenses of trough cross-stratification, mainly at the top. Elsewhere, the top is totally bioturbated and ferruginized (Figs. 7 and 13). Moreover, a patch of ferruginized fossil turf was found at the top of the ZMb sandstone in the northern part of the quarry at locality 5 (Fig. 14C, D).

The contact of the ZMb and CFm is exposed at localities $1 \mathrm{~B}, 4,5,9$ and 12 (Fig. 1), with only vestiges of the CFm exposed at the first and third locality, but with up to $12 \mathrm{~m}$ thickness exposed at the remaining three localities. The CFm succession in each outcrop is different in respect to lithological composition, lithofacies distribution and sediment features (Fig. 7). In all outcrops, the succession commences with a claystone- to mudstone-dominated interval, $1.3 \mathrm{~m}$ to several metres thick, representing the $\mathrm{NMb}$ (Fig. 3). Its subordinate components are siltstone, thin-bedded sandstone, one to three thin local coal beds (Figs. 7, 15A, 16D) and a few thin siderite layers (Figs. 4, 7 

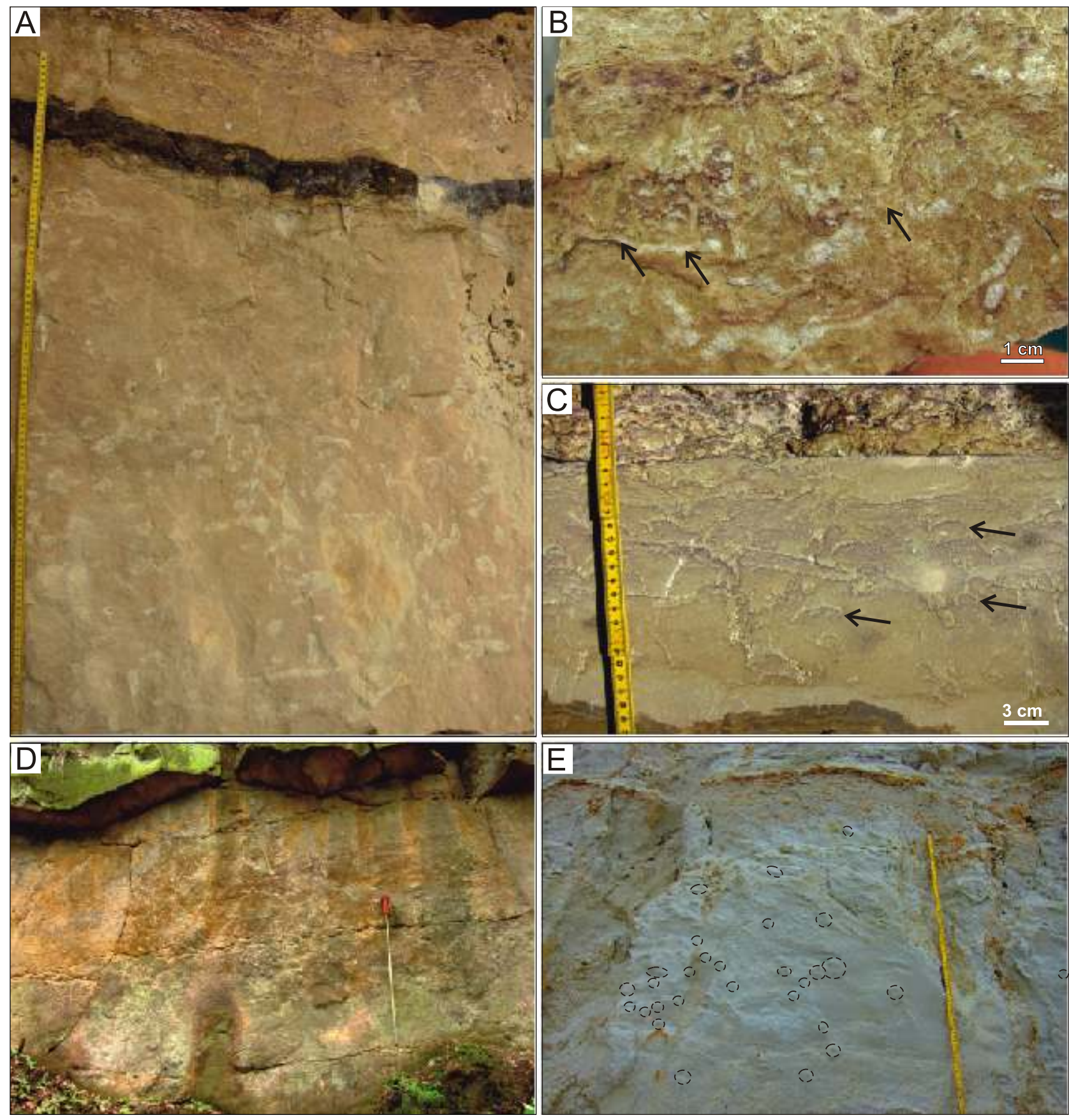

Fig. 10. Variable bioturbation intensity in vertical sections of sandstone beds in the erkowice Member

A - bioturbation with distinct Ophiomorpha in the lower part, and ferruginization in the top part of sandstone bed terminating the erkowice Member at locality 5 (Wartowice), photography August 2012; B - suspected plant-root structures (arrows) at the top of sandstone bed in the upper part of the erkowice Member, locality 5 (Wartowice), photography August 2011; C - upwards increasing bioturbation in sandstone bed with Ophiomorpha lacking burrow-floor lining (arrows), locality 5 (Wartowice), photography August 2012; D - distribution of Ophiomorpha burrows (dashed outlines) in a sandstone bed with erosional top, locality (Kotliska), photography May 2013; $\mathbf{E}$ - ichnofabric and bioturbation (increasing from BI = 0 to 6 ) in a mudstone-covered sandstone bed, with Ophiomorpha (circled) in the deepest part and with the mudstone cover indicating temporal stagnation of a sandy seafloor, locality 12 (Osiecznica), photography April 2013; localities as in Table 1 and their map location in Figure1B

and $16 \mathrm{~F})$. The CFm at locality 5 commences with a greyish white to brownish red mudstone, up to $30 \mathrm{~cm}$ thick, that passes upwards into a dark grey to dark green mudstone with a thin patch of coal or into a wedge of very fine-grained sandstone, up to $50 \mathrm{~cm}$ thick, which in the northern part of the quarry rests directly on the ZMb sandstone (Fig. 7). The mudstone as well as the sandstone show plant-root structures (rhizoliths and rhizocretions; Fig. 14B, E, F). At localities 4, 9 and 12 (Fig. 1), the CFm commences with a clayey mudstone containing locally a few thin sandstone interlayers. The mudstone is orange, $30 \mathrm{~cm}$ thick and overlain by dark grey to black clayey mudstone at locality 4; greyish white to reddish orange, $30-55 \mathrm{~cm}$ thick and 

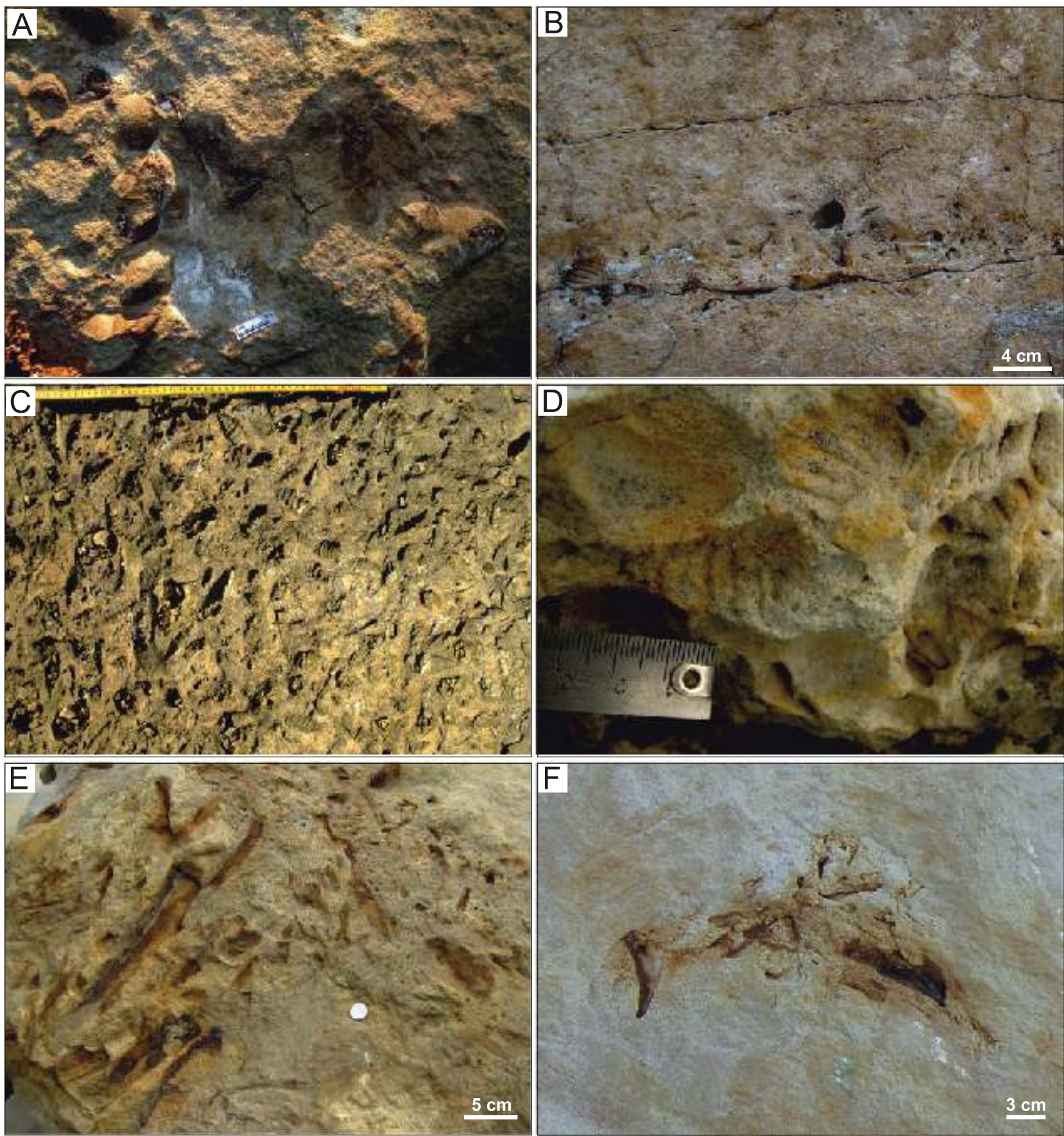

Fig. 11. Casts and imprints of body fossils in sandstones of the erkowice Member

A - shell lag recorded by casts of bivalve shells, locality 5 (Wartowice), photography October 2010; B - sandstone with a horizon rich in voids after shelly fauna, locality 3 (Gaszów), photography April 2009; C - shell lag dominated by imprints of the gastropod Nerinea bicincta on the top of sandstone bed, locality 4 ( eliszów), photography October 2010; D -imprints of clustered mollusc shells, locality 3 (Gaszów), photography April 2009; E - casts of ?vertebrate bones, locality 8 ( erkowice), photography July 2007; F - casts of vertebrate bones, locality 5 (Wartowice), photography October 2010; localities as in Table 1 and their map location in Figure 1B

overlain by dark grey silty mudstone and sandy siltstone at locality 9; greyish white to orange, $20-25 \mathrm{~cm}$ thick and overlain by to dark grey to blackish clayey mudstone at locality 12 . Coal layers are underlain by clayey to muddy seat earth (palaeosol of vertisol and histosol type; see Retallack, 1988) with locally plant-root structures (Fig. 15A, C).
The higher part of the CFm succession is an alternation of mainly fine- to medium-grained sandstones and mudstones, with laterally varying proportions and lithofacies. Direct observations were made mainly in the lower portion of these deposits, particularly at locality 5 , because the higher portion was hardly accessible, poorly exposed and weathered. Sandstone-dominated inter- 

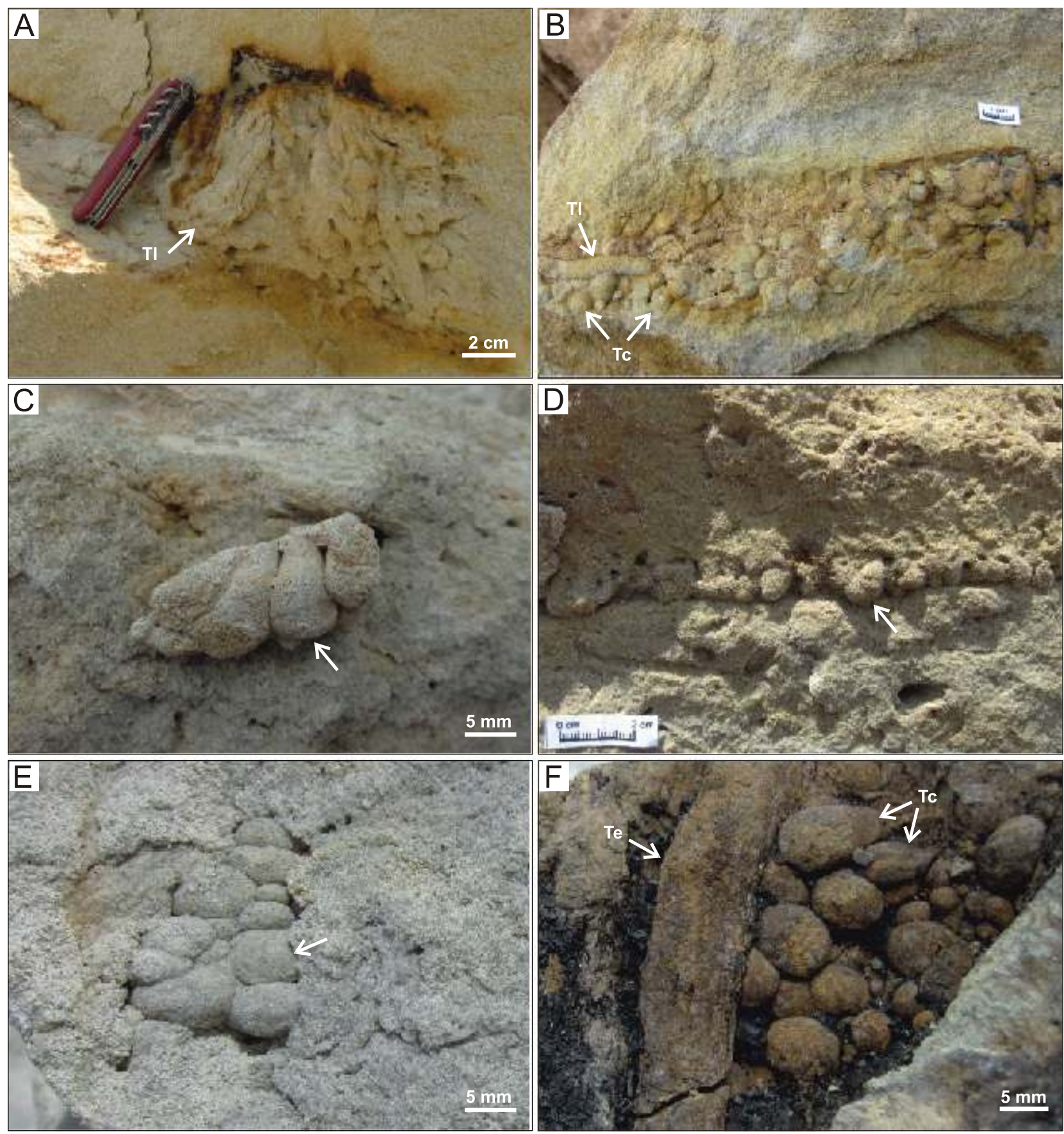

\section{Fig. 12. Teredolites in the deposits studied}

A - cluster of Teredolites longissimus? (TI) casts in a drifted tree trunk in sandstone of the erkowice Member, locality 8 ( erkowice), photography July 2008; B - casts of Teredolites clavatus (TC) and T. longissimus (TI) in a wood fragment in sandstone of the erkowice Member, locality 5 (Wartowice), photography May 2013; C - casts of Teredolites clavatus in sandstone in the top part of the erkowice Member, locality 12 (Osiecznica), photography August 2012; D - casts of Teredolites clavatus enclosed in sandstone in the top part of the erkowice Member (Osiecznica) photography August 2012; E - casts of Teredolites clavatus in sandstone in the top part of the erkowice Member, locality 12 (Osiecznica), photography August 2012; F - casts of Teredolites clavatus (Tc) and Teichichnus isp.

(Te) in the top part of a thin coal bed in the Czerna Formation, locality 9 (Rakowice Małe), photography May 2008; localities as in Table 1 and their map location in Figure 1B

vals, often coarsening upwards, alternate with fining- to coarsening-upwards, mudstone-dominated heterolithic intervals (Fig. 7). The latter show planar parallel stratification in sandstone layers as well as heterolithic flaser, wavy and lenticular bedding where non-bioturbated or weakly bioturbated. Where strongly biotur- bated $(\mathrm{BI}=5$ to 6$)$, they show a mottled structure with relics of faint, discontinuous, wispy lamination and a ripple cross-lamination or parallel lamination in sandstone layers, with a range of trace fossils. Bidirectional ripples were recognized at locality 9 , where the exposed part of the CFm is dominated by dark grey to 
List and taxonomic characteristics of trace fossils recognized in the upper part of the erkowice Member (ZMb) and the lowest part of the Czerna Formation (CFm); plant-root traces not included

\begin{tabular}{|c|c|c|c|c|c|}
\hline $\begin{array}{l}\text { Ichnogenus/ichnospe } \\
\text { cies (with photo- } \\
\text { graph references) }\end{array}$ & Short description & Ethology & $\begin{array}{l}\text { Inferred } \\
\text { producer }\end{array}$ & Occurrence & Key references \\
\hline $\begin{array}{c}\text { Arenicolites sparsus } \\
\text { Fig. } 37 \mathrm{~A}\end{array}$ & $\begin{array}{l}\text { Vertical, U-shaped tubular burrows } \\
\text { without spreite between limbs. Tube di- } \\
\text { ameter } 4 \mathrm{~mm} \text {, thinly lined. Limb spacing } \\
\text { up to } 20 \mathrm{~mm}\end{array}$ & Domichnion & Polychaetes & $\begin{array}{l}\text { Found as one fully preser- } \\
\text { ved specimen and several } \\
\text { fragments in sandstones of } \\
\text { a heterolithic package in } \\
\text { the CFm at locality } 9\end{array}$ & $\begin{array}{c}\text { Fürsich (1974), } \\
\text { Rindsberg and } \\
\text { Kopaska-Merkel } \\
\text { (2005), } \\
\text { Knaust (2017) }\end{array}$ \\
\hline $\begin{array}{l}\text { ?Arenicolites isp. } \\
\text { Fig. 15F in } \\
\text { Leszczyński (2010) }\end{array}$ & $\begin{array}{l}\text { Vertical, U-shaped tubular burrows with- } \\
\text { out spreite between limbs. Tube diame- } \\
\text { ter } 5 \mathrm{~mm} \text {. Limb spacing up to } 30 \mathrm{~mm}\end{array}$ & Domichnion & $\begin{array}{c}\text { Poly- } \\
\text { chaetes, } \\
\text { crustaceans }\end{array}$ & $\begin{array}{l}\text { Found as } 3 \text { fragmentarily } \\
\text { preserved specimens in } \\
\text { one sandstone bed in the } \\
\text { CFm at locality } 9\end{array}$ & $\begin{array}{c}\text { Fürsich (1974), } \\
\text { Rindsberg and } \\
\text { Kopaska-Merkel } \\
\text { (2005), } \\
\text { Knaust (2017) }\end{array}$ \\
\hline $\begin{array}{c}\text { Asterosoma } \\
\text { ludwigae } \\
\text { Fig. } 33 \\
\text { Leszczyński } \\
\text { (2010: fig. 14D-F) }\end{array}$ & $\begin{array}{l}\text { Horizontal to inclined burrows consist- } \\
\text { ing of bulbs that bud out from a circular } \\
\text { or elliptical tubular stem in a dichoto- } \\
\text { mous to fan-like pattern. Stems hori- } \\
\text { zontal, some bending up to vertical, } \\
10-15 \mathrm{~mm} \text { in diameter, with a massive } \\
\text { or faintly concentrically laminated infill. } \\
\text { Bulbs } 5-30 \mathrm{~mm} \text { in diameter, } 50-80 \mathrm{~mm} \\
\text { long, horizontal to oblique }\end{array}$ & Fodinichnion & Polychaetes & $\begin{array}{l}\text { Found as many specimens } \\
\text { in muddy sandstone of the } \\
\text { CFm at locality } 9\end{array}$ & $\begin{array}{l}\text { Schlirf (2000), } \\
\text { Knaust (2017) }\end{array}$ \\
\hline $\begin{array}{c}\text { Asterosoma coxii } \\
\text { Fig. 27E, F }\end{array}$ & $\begin{array}{l}\text { Bunch of club-shaped and bud-shaped } \\
\text { sandbodies, smooth walled, budding } \\
\text { out from a bedding-oblique stem. Inter- } \\
\text { nal structure not recognized }\end{array}$ & Fodinichnion & Polychaetes & $\begin{array}{l}\text { Found as two specimens } \\
7-10 \mathrm{~mm} \text { thick and up to } 20 \\
\mathrm{~mm} \text { long on soles of sand- } \\
\text { stone beds in the ZMb at } \\
\text { localities } 6 \text { and } 8\end{array}$ & $\begin{array}{c}\text { Seilacher (2007), } \\
\text { Knaust (2017) }\end{array}$ \\
\hline $\begin{array}{l}\text { ?Asterosoma isp. } \\
\text { Fig. } 34 \mathrm{~A}, \mathrm{~B}\end{array}$ & $\begin{array}{l}\text { Structures visible in vertical bed sections } \\
\text { as stacks of horizontal to inclined, } \\
\text { slightly concave to convex laminae, up } \\
\text { to } 4 \mathrm{~cm} \text { long and } 1-2 \mathrm{~cm} \text { high; some con- } \\
\text { centrically laminated, up to } 1.5 \mathrm{~cm} \text { in di- } \\
\text { ameter, with a massive core. Can be } \\
\text { confused with Teichichnus isp. or } \\
\text { Cylindrichnus isp. }\end{array}$ & Fodinichnion & Polychaetes & $\begin{array}{l}\text { Found as mass occurren- } \\
\text { ces in some parts of hetero- } \\
\text { lithic deposits of the CFm at } \\
\text { locality } 9\end{array}$ & \\
\hline $\begin{array}{l}\text { Aulichnites } \\
\text { parkerensis } \\
\text { Fig. } 27 \mathrm{~A}, \mathrm{~B}\end{array}$ & $\begin{array}{l}\text { Bilobate, unlined, unbranched, actively } \\
\text { filled convex, unornamented epirelief, } \\
5-6 \mathrm{~mm} \text { wide, sinuous to straight. Nar- } \\
\text { row median furrow }\end{array}$ & Repichnion & Gastropods & $\begin{array}{l}\text { Found as many specimens } \\
\text { on top surface of sand- } \\
\text { stone bed in the ZMb at lo- } \\
\text { cality } 9\end{array}$ & $\begin{array}{c}\text { Fenton and } \\
\text { Fenton (1937), } \\
\text { Frey and Howard } \\
(1990)\end{array}$ \\
\hline $\begin{array}{c}\text { Biformites insolitus } \\
\text { Fig. } 27 \mathrm{C}\end{array}$ & $\begin{array}{l}\text { Narrow, vermiform, slightly tapering, hori- } \\
\text { zontal, straight to curved structures } 16 \\
\text { mm long with delicate, ideally bi-symmet- } \\
\text { rically arranged, transverse elongate or } \\
\text { polygonal to round, positive or negative } \\
\text { protuberances. Sets of transverse ribs } \\
\text { and furrows in the burrow wider part, } \\
\text { faintly dissected along its axis } \\
\end{array}$ & Cubichnion & Ophiuroids & $\begin{array}{l}\text { Found as one specimen on } \\
\text { sandstone bed sole in ZMb } \\
\text { at locality } 9\end{array}$ & $\begin{array}{l}\text { Häntzschel } \\
(1975), \\
\text { Schlirf (2012) }\end{array}$ \\
\hline $\begin{array}{c}\text { Chondrites intricatus } \\
\text { Fig. 37B } \\
\text { Leszczyński } \\
\text { (2010: fig 14E, F) }\end{array}$ & $\begin{array}{l}\text { Clusters of dendritic, straight to widely } \\
\text { curved endoreliefs made of silt, very } \\
\text { fine sand or mud, showing acute irregu- } \\
\text { lar branching. Burrows } \sim 1 \text { mm wide, } \\
10-20 \mathrm{~mm} \text { long, showing two orders of } \\
\text { dichotomous branching. In vertical sec- } \\
\text { tions seen as clusters of light grey ellip- } \\
\text { tical spots and veins }\end{array}$ & $\begin{array}{c}\text { Chemichnion } \\
\text { agrichnion }\end{array}$ & $\begin{array}{l}\text { ?Worm-like } \\
\text { animals }\end{array}$ & $\begin{array}{l}\text { Several occurrences in } \\
\text { coaly mudstone in the CFm } \\
\text { at locality } 9\end{array}$ & $\begin{array}{l}\text { Fu (1991), } \\
\text { Uchman (1998), } \\
\text { Seilacher (2007) }\end{array}$ \\
\hline $\begin{array}{l}\text { ?Curvolithus isp. } \\
\text { Fig. } 27 \mathrm{C}, \mathrm{E}\end{array}$ & $\begin{array}{l}\text { Horizontal, bilobate, delicately winding } \\
\text { trace fossil, } 7 \mathrm{~mm} \text { wide and up to } 4 \mathrm{~cm} \\
\text { long, consisting of two lateral lobes sep- } \\
\text { arated by similarly wide furrow }\end{array}$ & Repichnion & Gastropods & $\begin{array}{l}\text { Several specimens found } \\
\text { on sandstone bed sole in } \\
\text { the ZMb at locality } 9\end{array}$ & $\begin{array}{l}\text { Fritsch (1908), } \\
\text { Fillion and } \\
\text { Pickerill (1990) }\end{array}$ \\
\hline $\begin{array}{l}\text { Cylindrichnus } \\
\text { concentricus } \\
\text { Fig. 36A-C }\end{array}$ & $\begin{array}{l}\text { Gently curved, unbranched, slightly coni- } \\
\text { cal, downwards tapering burrows, nearly } \\
\text { circular in cross-section, 5-15 mm in di- } \\
\text { ameter, with concentrically lined infill and } \\
\text { faint internal layering. Burrows vertically } \\
\text { arcuate to oblique }\end{array}$ & Domichnion & $\begin{array}{c}\text { Terebellid } \\
\text { polychaetes, } \\
\text { entero- } \\
\text { pneusts }\end{array}$ & $\begin{array}{l}\text { Found as many specimens } \\
\text { in heterolithic deposits of } \\
\text { the CFm at locality } 9\end{array}$ & $\begin{array}{l}\text { Belaústegui and } \\
\text { Gibert (2013), } \\
\text { Frey and Howard } \\
\text { (1985); Ekdale } \\
\text { and Harding } \\
\text { (2015) }\end{array}$ \\
\hline $\begin{array}{c}\text { ?Diplocraterion isp. } \\
\text { Fig. } 25 \mathrm{~A}\end{array}$ & $\begin{array}{l}\text { Vertical U-shaped spreited burrow with } \\
\text { limb diameter } 7-10 \mathrm{~mm} \text { and limb spacing } \\
15-70 \mathrm{~mm} \text {; occurs as dumbbell-shaped } \\
\text { structures made of sand similar to host } \\
\text { sediment. U-shaped limbs connected by } \\
\text { spreite resemble Diplocraterion }\end{array}$ & Domichnion & Polychaetes & $\begin{array}{l}\text { Found as several speci- } \\
\text { mens on sandstone bed } \\
\text { sole in a block from the ZMb } \\
\text { at locality } 9\end{array}$ & $\begin{array}{l}\text { Fürşich (1974); } \\
\text { Simo and } \\
\text { Olšavský (2007); } \\
\text { Knaust (2017) }\end{array}$ \\
\hline $\begin{array}{l}\text { Helminthopsis abeli } \\
\text { Figs. } 27 \mathrm{E} \\
\text { and } 37 \mathrm{C}, \mathrm{D}\end{array}$ & $\begin{array}{l}\text { Horizontal, irregularly winding, unbran- } \\
\text { ched, string-sized, smooth-walled, mud- } \\
\text {-lined trace fossil 1.8-2.5 mm wide }\end{array}$ & Fodinichnion & $\begin{array}{l}\text { ?Poly- } \\
\text { chaetes }\end{array}$ & $\begin{array}{l}\text { Found as several speci- } \\
\text { mens on sandstone bed so- } \\
\text { les in the ZMb locality } 8 \text { and } \\
\text { in numerous cross-sections } \\
\text { in heterolithic deposits of } \\
\text { CFm at locality } 9\end{array}$ & $\begin{array}{c}\text { Ksia kiewicz } \\
\text { (1977), } \\
\text { Uchman (1998) }\end{array}$ \\
\hline $\begin{array}{l}\text { ?Lockeia isp. } \\
\text { Fig. 27B-D }\end{array}$ & $\begin{array}{l}\text { Hypichnial, almond-outlined protrusion } \\
0.2-10 \mathrm{~mm} \text { long, } 2-4 \mathrm{~mm} \text { wide and } 2-6 \\
\mathrm{~mm} \text { high }\end{array}$ & Cubichnion & Bivalves & $\begin{array}{l}\text { Found as several speci- } \\
\text { mens on sandstone bed so- } \\
\text { le in } \mathrm{ZMb} \text { at locality } 5\end{array}$ & $\begin{array}{c}\text { Maples and West } \\
\text { (1989), } \\
\text { Schlirf (2000) }\end{array}$ \\
\hline
\end{tabular}


Tab. 1 cont.

\begin{tabular}{|c|c|c|c|c|c|}
\hline $\begin{array}{c}\text { Ichnogenus/ichnospe } \\
\text { cies (with photo- } \\
\text { graph references) } \\
\end{array}$ & Short description & Ethology & $\begin{array}{l}\text { Inferred } \\
\text { producer }\end{array}$ & Occurrence & Key references \\
\hline $\begin{array}{l}\text { aff. Macaronichnus } \\
\text { isp. } \\
\text { Figs. } 28 \mathrm{~A} \text { and } 36 \mathrm{E}\end{array}$ & $\begin{array}{l}\text { Cylindrical, straight to winding, unbran- } \\
\text { ched, horizontal burrows, 3-6 mm in di- } \\
\text { ameter, without distinct mantle of dark } \\
\text { mineral grains. In cross-section seen as } \\
\text { circular or elongate spots parallel or } \\
\text { slightly oblique to bedding plane. In verti- } \\
\text { cal section seen as strings of densely dis- } \\
\text { tributed, round, elliptical to elongate } \\
\text { spots enriched in dark substance }\end{array}$ & Fodinichnion & $\begin{array}{l}\text { Endobenthic } \\
\text { polychaetes }\end{array}$ & $\begin{array}{l}\text { Sporadic occurrences in } \\
\text { the ZMb at locality } 12 \text { and } \\
\text { in the CFm at locality } 9\end{array}$ & $\begin{array}{c}\text { Clifton and } \\
\text { Thompson (1978), } \\
\text { Nara and Seike } \\
\text { (2004), } \\
\text { Seike (2007), } \\
\text { Seike et al. (2011) }\end{array}$ \\
\hline $\begin{array}{c}\text { ?Nereites irregularis } \\
\text { Fig. } 27 \mathrm{~F}\end{array}$ & $\begin{array}{l}\text { Endichnial and epichnial, irregularly } \\
\text { meandering, horizontal to subhorizontal } \\
\text { narrow-gauged tubes, void or infilled, } \\
\text { with signs of transverse segmentation. } \\
\text { Epichnial trace forms furrows } 1-3 \mathrm{~mm} \\
\text { wide, incised about } 1-1.5 \mathrm{~mm} \text { in sub- } \\
\text { strate, arcuate and filled with flattened } \\
\text { cylinders. Delicate transverse striation } \\
\text { is visible on the bottom of some furrows } \\
\end{array}$ & Fodinichnion & $\begin{array}{l}\text { Arthropods } \\
\text { or annelids }\end{array}$ & $\begin{array}{l}\text { One specimen found in } \\
\text { sandstone of the ZMb at lo- } \\
\text { cality } 5\end{array}$ & $\begin{array}{c}\text { Ksia kiewicz } \\
\text { (1977), } \\
\text { Uchman (1998), } \\
\text { Wetzel (2002), } \\
\text { Martin and } \\
\text { Rindsberg (2007) }\end{array}$ \\
\hline $\begin{array}{c}\text { Ophioichnus isp. } \\
\text { Fig. } 29 \mathrm{~A}\end{array}$ & $\begin{array}{l}\text { Bundles of bowed furrows with slightly } \\
\text { widened outer endings, arranged in rows } \\
\text { and partly overlapped. Furrows ar- } \\
\text { ranged fan-like, with some crisscrossing }\end{array}$ & Repichnion & Ophiuroids & $\begin{array}{l}\text { Found at sandstone bed } \\
\text { top in a loose block from } \\
\text { the ZMb at locality } 5\end{array}$ & $\begin{array}{l}\text { cf. Belaústegui et } \\
\text { al. (2017: fig. 5), } \\
\text { Bell (2004) }\end{array}$ \\
\hline $\begin{array}{l}\text { Ophiomorpha } \\
\text { annulata } \\
\text { Fig. } 31 \mathrm{~F}\end{array}$ & $\begin{array}{l}\text { Simple to complex burrow systems con- } \\
\text { sisting of straight to slightly curved, } \\
\text { branched, horizontal to vertical bur- } \\
\text { rows, } 8 \mathrm{~mm} \text { in diameter, lined with } \\
\text { evenly spaced rows of elliptical pellets } \\
\text { (mud-marked ribs) transverse to burrow } \\
\text { axis. End-to-end pellets form more or } \\
\text { less continuous rings or annulations } \\
\text { around burrow segments }\end{array}$ & $\begin{array}{l}\text { Domichnion, } \\
\text { fodinichnion }\end{array}$ & $\begin{array}{l}\text { Crusta- } \\
\text { ceans }\end{array}$ & $\begin{array}{l}\text { Found as two specimens } \\
\text { (tunnel moulds) in sand- } \\
\text { stone in the CFm at local- } \\
\text { ity } 9\end{array}$ & $\begin{array}{l}\text { Frey et al. (1978), } \\
\text { Howard and Frey } \\
(1984) \text {, } \\
\text { Frey and Howard } \\
\text { (1985, 1990), } \\
\text { Uchman (1998) }\end{array}$ \\
\hline $\begin{array}{l}\text { Ophiomorpha } \\
\text { nodosa } \\
\text { Figs. } 15 \mathrm{E}, 18-20 \\
\text { and } 31 \mathrm{~A}, \mathrm{C}-\mathrm{E}\end{array}$ & $\begin{array}{l}\text { Simple to complex burrow systems con- } \\
\text { sisting of straight to slightly curved, bran- } \\
\text { ched positive epireliefs, horizontal to } \\
\text { vertical endoreliefs or positive hypore- } \\
\text { liefs, lined with regularly to irregularly dis- } \\
\text { tributed discoid, ovoid, round, polygonal, } \\
\text { conical or irregular pellets. Burrows main- } \\
\text { ly horizontal, } 5-40 \mathrm{~mm} \text { in diameter, sho- } \\
\text { wing a smooth-walled sand core lined } \\
\text { with densely to sparsely distributed pel- } \\
\text { lets and chaotic muddy sediment. Lining } \\
\text { type varies within single burrows } \\
\end{array}$ & $\begin{array}{l}\text { Domichnion, } \\
\text { fodinichnion }\end{array}$ & $\begin{array}{l}\text { Crusta- } \\
\text { ceans }\end{array}$ & $\begin{array}{l}\text { Most extensive in sandsto- } \\
\text { nes of the ZMb and rare in } \\
\text { the CFm at localities } 5 \text { and } \\
9\end{array}$ & $\begin{array}{l}\text { Frey et al. (1978), } \\
\text { Howard and Frey } \\
\text { (1984), } \\
\text { Frey and Howard } \\
\text { (1985, 1990), } \\
\text { Uchman (1998), } \\
\text { Blisset and } \\
\text { Pickerill (2004), } \\
\text { Gani et al. (2007) }\end{array}$ \\
\hline $\begin{array}{l}\text { Ophiomorpha } \\
\text { ?nodosa } \\
\text { Fig. } 21\end{array}$ & $\begin{array}{l}\text { Systems of horizontal, winding to cur- } \\
\text { ved, densely branched cylindrical bur- } \\
\text { rows, } 10 \mathrm{~mm} \text { in diameter, lined regularly } \\
\text { with densely distributed round-shaped } \\
\text { pellets, locally paired }\end{array}$ & $\begin{array}{l}\text { Domichnion, } \\
\text { fodinichnion }\end{array}$ & $\begin{array}{l}\text { Crusta- } \\
\text { ceans }\end{array}$ & $\begin{array}{l}\text { Found as two small sys- } \\
\text { tems at sandstone bed top } \\
\text { in the ZMb at locality } 5\end{array}$ & $\begin{array}{l}\text { Frey et al. (1978), } \\
\text { Howard and Frey } \\
\text { (1984), Uchman } \\
\text { (1998), Blisset and } \\
\text { Pickerill (2004), } \\
\text { Gani et al. (2007) }\end{array}$ \\
\hline $\begin{array}{c}\text { Ophiomorpha isp. } \\
\text { Fig. } 22\end{array}$ & $\begin{array}{l}\text { Horizontal to vertical, branched tubular } \\
\text { burrows } 30-40 \mathrm{~mm} \text { in diameter, lined } \\
\text { with irregularly distributed conical mud } \\
\text { pellets Burrow-fill with a rough, pitted } \\
\text { knobbly surface. Occurs as moulds and } \\
\text { casts }\end{array}$ & $\begin{array}{l}\text { Domichnion, } \\
\text { fodinichnion }\end{array}$ & $\begin{array}{l}\text { Crusta- } \\
\text { ceans }\end{array}$ & $\begin{array}{l}\text { Found as many specimens } \\
\text { in one sandstone bed in } \\
\text { the ZMb at locality } 10\end{array}$ & $\begin{array}{l}\text { Frey et al. (1978), } \\
\text { Howard and Frey } \\
\qquad(1984)\end{array}$ \\
\hline $\begin{array}{l}\text { ?Palaeophycus } \\
\text { heberti } \\
\text { Figs. 35C, D, 36C } \\
\text { and 33C }\end{array}$ & $\begin{array}{l}\text { Gently curved, unbranched, cylindrical } \\
\text { to subcylindrical burrows, } 5-15 \mathrm{~mm} \text { in } \\
\text { diameter, horizontal and oblique, with a } \\
\text { lining } 1.5-3 \mathrm{~mm} \text { thick }\end{array}$ & Fodinichnion & ?Annelids & $\begin{array}{l}\text { Found as many specimens } \\
\text { in vertical section in the } \\
\text { CFm at locality } 9\end{array}$ & $\begin{array}{c}\text { Pemberton and } \\
\text { Frey (1982), } \\
\text { Howard and Frey } \\
\text { (1984), } \\
\text { Nara (2006) }\end{array}$ \\
\hline $\begin{array}{l}\text { Palaeophycus } \\
\text { sulcatus } \\
\text { Fig. } 30 \mathrm{~B}\end{array}$ & $\begin{array}{l}\text { Horizontal, unbranched, subcylindrical } \\
\text { burrows, } 8-10 \mathrm{~mm} \text { thick and up to } 20 \\
\mathrm{~cm} \text { long, thinly lined and ornamented } \\
\text { with sharp, anastomosing, thread-like } \\
\text { striations }\end{array}$ & Fodinichnion & Annelids & $\begin{array}{l}\text { Found as several speci- } \\
\text { mens on sandstone bed } \\
\text { soles in the ZMb at locality } 5\end{array}$ & $\begin{array}{l}\text { Pemberton and } \\
\text { Frey (1982) }\end{array}$ \\
\hline $\begin{array}{l}\text { Palaeophycus } \\
\text { ?tubularis } \\
\text { Fig. 33E }\end{array}$ & $\begin{array}{l}\text { Predominantly horizontal, straight to } \\
\text { slightly curved tubular burrows, 3-7 } \mathrm{mm} \\
\text { in diameter, thinly but distincly lined }\end{array}$ & Fodinichnion & $\begin{array}{l}\text { Endobenthic } \\
\text { ?worm-like } \\
\text { animals }\end{array}$ & $\begin{array}{l}\text { Found as short segments } \\
\text { on sandstone parting and } \\
\text { bedding surfaces in the } \\
\text { CFm at locality } 9\end{array}$ & $\begin{array}{l}\text { Pemberton and } \\
\text { Frey (1982) }\end{array}$ \\
\hline $\begin{array}{l}\text { ?Palaeophycus isp. } \\
\text { Figs. 33B, D, E } \\
\text { and } 35 A, B\end{array}$ & $\begin{array}{l}\text { Bedding-parallel to oblique, endichnial } \\
\text { and epichnial tubular burrows, } \\
3-15 \mathrm{~mm} \text { in diameter, made of sand } \\
\text { with a thin }(0.5-1 \mathrm{~mm}) \text { mud lining }\end{array}$ & Fodinichnion & $\begin{array}{c}\text { Endobenthic } \\
\text { ?worm-like } \\
\text { animals }\end{array}$ & $\begin{array}{l}\text { Found as many specimens } \\
\text { in vertical section in muddy } \\
\text { sandstones of the CFm at } \\
\text { locality } 9\end{array}$ & $\begin{array}{l}\text { Pemberton and } \\
\text { Frey (1982) }\end{array}$ \\
\hline $\begin{array}{c}\text { Planolites } \\
\text { beverleyensis } \\
\text { Figs. } 27 \mathrm{C} \text { and } 30 \mathrm{~B}\end{array}$ & $\begin{array}{l}\text { Relatively large, smooth, straight to gently } \\
\text { curved or undulose cylindrical burrows } \\
\text { with irregular diameter of up to 5-6 mm. } \\
\text { Unbranched, made of massive sand, with } \\
\text { smooth to slightly irregular walls }\end{array}$ & Fodinichnion & $\begin{array}{l}\text { Endobenthic } \\
\text { worm-like } \\
\text { animals }\end{array}$ & $\begin{array}{l}\text { Found as many specimens } \\
\text { on bedding surface in } \\
\text { many sandstone blocks of } \\
\text { the ZMb }\end{array}$ & $\begin{array}{l}\text { Pemberton and } \\
\text { Frey (1982), } \\
\text { Uchman (1995) }\end{array}$ \\
\hline $\begin{array}{l}\text { ?Planolites isp. } \\
\text { Fig. 33D, E }\end{array}$ & $\begin{array}{l}\text { Smooth-walled, straight to gently cur- } \\
\text { ved or undulose, unbranched, horizon- } \\
\text { tal to oblique cylindrical burrows, } \\
3-15 \mathrm{~mm} \text { in diameter, made of sand } \\
\text { and unlined }\end{array}$ & Fodinichnion & $\begin{array}{l}\text { Endobenthic } \\
\text { worm-like } \\
\text { animals }\end{array}$ & $\begin{array}{l}\text { Found in vertical section } \\
\text { exclusively in sandstones } \\
\text { and mudstones of the CFm } \\
\text { at locality } 9\end{array}$ & $\begin{array}{l}\text { Pemberton and } \\
\text { Frey (1982) }\end{array}$ \\
\hline
\end{tabular}


Tab. 1 cont

\begin{tabular}{|c|c|c|c|c|c|}
\hline $\begin{array}{c}\text { Ichnogenus/ichnospe } \\
\text { cies } \\
\text { (with photograph ref- } \\
\text { erences) }\end{array}$ & Short description & Ethology & $\begin{array}{l}\text { Inferred } \\
\text { producer }\end{array}$ & Occurrence & Key references \\
\hline $\begin{array}{c}\text { ?Rosselia isp. A } \\
\text { Fig. } 28 \mathrm{C}, \mathrm{D}\end{array}$ & $\begin{array}{l}\text { Vertical to subvertical spindle-shaped } \\
\text { burrows up to } 25 \mathrm{~cm} \text { long, circular to ir- } \\
\text { regularly oval in cross-section, at least } \\
\text { partly layered concentrically around cen- } \\
\text { tral to excentral tube. Spindles up to } 10 \\
\text { mm in diameter, crowded in the burrow } \\
\text { upper part and tapering downwards, co- } \\
\text { alescing into vertical or subvertical, } \\
\text { curved basal shafts that extend beneath } \\
\text { as a narrow slightly curved shaft. Spin- } \\
\text { dles and their internal layering often } \\
\text { poorly visible due to homogeneus infill } \\
\text { same as the host quartz arenite }\end{array}$ & $\begin{array}{l}\text { Fodinichnion } \\
\text { domichnion }\end{array}$ & $\begin{array}{c}\text { Terebellid } \\
\text { polychaetes }\end{array}$ & $\begin{array}{l}\text { Found as crowded in the } \\
\text { top part of one sandstone } \\
\text { bed covered by completely } \\
\text { bioturbated mudstone in } \\
\text { the ZMb at locality } 12\end{array}$ & $\begin{array}{l}\text { Howard and Frey } \\
\text { (1984), Frey and } \\
\text { Howard (1985), } \\
\text { Knaust (2017); } \\
\text { similar burrows } \\
\text { were classified by } \\
\text { Bromley and } \\
\text { Uchman (2003) as } \\
\text { Asterosoma isp. }\end{array}$ \\
\hline $\begin{array}{l}\text { ?Rosselia isp. B } \\
\text { Fig. 36F }\end{array}$ & $\begin{array}{l}\text { Horn-shaped, unbranched vertical to } \\
\text { oblique endorelief, up to } 15 \mathrm{~mm} \text { in diam- } \\
\text { eter and } 35 \mathrm{~mm} \text { high, filled with faint } \\
\text { laminae of mud and sand reminiscent of } \\
\text { concentric sheaths }\end{array}$ & $\begin{array}{l}\text { Fodinichnion } \\
\text { domichnion }\end{array}$ & $\begin{array}{c}\text { Terebellid } \\
\text { polychaetes }\end{array}$ & $\begin{array}{l}\text { Found as several spindle- } \\
\text {-shaped specimens in the } \\
\text { CFm at locality } 9\end{array}$ & $\begin{array}{l}\text { Howard and Frey } \\
\text { (1984), Frey and } \\
\text { Howard (1985), } \\
\text { Coates and } \\
\text { MacEachern } \\
\text { (2007), Miller and } \\
\text { Aalto (2008), } \\
\text { Knaust (2017) } \\
\end{array}$ \\
\hline $\begin{array}{l}\text { ?Schaubcylindrich- } \\
\text { nus isp. } \\
\text { Fig. 37D } \\
\text { Leszczyński } \\
\text { (2010: fig. 13C, E) }\end{array}$ & $\begin{array}{l}\text { Straight, bedding-oblique, unbranched } \\
\text { endorelief recorded as clusters of con- } \\
\text { gruent sand-lined tubes, } 7 \mathrm{~mm} \text { in diam- } \\
\text { eter, with relatively thick }(1.5 \mathrm{~mm} \text { ) light } \\
\text { colour lining. Resembles } S \text { freyi in lin- } \\
\text { ing type, but has tubes much larger and } \\
\text { only bedding-obliquely aligned }\end{array}$ & Domichnion & $\begin{array}{l}\text { Endobenthic } \\
\text { sessile } \\
\text { ?poly- } \\
\text { chaetes }\end{array}$ & $\begin{array}{l}\text { Found as several speci- } \\
\text { mens in heterolithic depos- } \\
\text { its of the CFm at locality } 9\end{array}$ & $\begin{array}{l}\text { Frey and Howard } \\
\text { (1981,1985), } \\
\text { Nara (2006) }\end{array}$ \\
\hline $\begin{array}{c}\text { aff. Scolicia strozzii } \\
\text { Fig. 25B }\end{array}$ & $\begin{array}{l}\text { Straight to slightly winding, hypichnial } \\
\text { bilobate ridge preserved as semi-relief, } \\
10-20 \mathrm{~mm} \text { wide and } \sim 10 \mathrm{~mm} \text { deep, with } \\
\text { a median groove. The ridge prominent } \\
\text { zones and the groove are more or less } \\
\text { arcuate in cross-section and their sur- } \\
\text { face tends to be corrugated }\end{array}$ & Fodinichnion & $\begin{array}{l}\text { Echinoids, } \\
\text { molluscs }\end{array}$ & $\begin{array}{l}\text { Found on sandstone bed } \\
\text { sole in as loose block from } \\
\text { the ZMb at locality } 8\end{array}$ & $\begin{array}{c}\text { Ksia kiewicz } \\
(1977), \\
\text { Uchman (1998) }\end{array}$ \\
\hline $\begin{array}{l}\text { ?Sinusichnus isp. } \\
\text { Figs. } 25 \mathrm{~A} \text { and } 28 \mathrm{~B}\end{array}$ & $\begin{array}{l}\text { Horizontal system of sinusoidal, mean- } \\
\text { dering and winding, branched vermi- } \\
\text { form burrows, } 4-10 \mathrm{~mm} \text { in diameter, } \\
\text { made of sand }\end{array}$ & Fodinichnion & $\begin{array}{l}\text { Decapod } \\
\text { or worm-like } \\
\text { animals }\end{array}$ & $\begin{array}{l}\text { Found in the ZMb sandsto- } \\
\text { ne as relief segments up to } \\
2 \mathrm{~cm} \text { long on parting surface } \\
\text { of sandstone block at local- } \\
\text { ity } 9 \text { and as cast on sand- } \\
\text { stone bed sole at locality } 8\end{array}$ & $\begin{array}{c}\text { Gibert (1996), } \\
\text { Belaústegui et al. } \\
(2014)\end{array}$ \\
\hline $\begin{array}{c}\text { ?Rosselia isp. A } \\
\text { Fig. 28C, D }\end{array}$ & $\begin{array}{l}\text { Vertical to subvertical spindle-shaped } \\
\text { burrows up to } 25 \mathrm{~cm} \text { long, circular to ir- } \\
\text { regularly oval in cross-section, at least } \\
\text { partly layered concentrically around cen- } \\
\text { tral to excentral tube. Spindles up to } 10 \\
\text { mm in diameter, crowded in the burrow } \\
\text { upper part and tapering downwards, co- } \\
\text { alescing into vertical or subvertical, } \\
\text { curved basal shafts that extend beneath } \\
\text { as a narrow slightly curved shaft. Spin- } \\
\text { dles and their internal layering often } \\
\text { poorly visible due to homogeneus infill } \\
\text { same as the host quartz arenite }\end{array}$ & $\begin{array}{c}\text { Fodinichnion } \\
\text { domichnion }\end{array}$ & $\begin{array}{c}\text { Terebellid } \\
\text { polychaetes }\end{array}$ & $\begin{array}{l}\text { Found as crowded in the } \\
\text { top part of one sandstone } \\
\text { bed covered by completely } \\
\text { bioturbated mudstone in } \\
\text { the ZMb at locality } 12\end{array}$ & $\begin{array}{c}\text { Howard and Frey } \\
\text { (1984), Frey and } \\
\text { Howard (1985), } \\
\text { Knaust (2017); } \\
\text { similar burrows } \\
\text { were classified by } \\
\text { Bromley and } \\
\text { Uchman (2003) as } \\
\text { Asterosoma isp. }\end{array}$ \\
\hline $\begin{array}{l}\text { Skolithos linearis } \\
\text { Fig. 35G } \\
\text { Leszczyński } \\
\text { (2010: fig. 16D) } \\
\end{array}$ & $\begin{array}{l}\text { Simple vertical, unbranched tubes, } \\
3-5 \mathrm{~mm} \text { in diameter and >50 } \mathrm{mm} \text { in } \\
\text { length, somewhat irregular, with an indis- } \\
\text { tinct wall and homogeneous sandy infill }\end{array}$ & Domichnion & $\begin{array}{l}\text { Annelids, } \\
\text { phoronids }\end{array}$ & $\begin{array}{l}\text { Found as many fragmenta- } \\
\text { rily preserved specimens } \\
\text { in a completely burrowed, } \\
\text { crumbled muddy sandsto- } \\
\text { ne of CFm at locality } 9\end{array}$ & $\begin{array}{c}\text { Fillion and } \\
\text { Pickerill (1990), } \\
\text { MacEachern et al. } \\
(2007 a)\end{array}$ \\
\hline $\begin{array}{l}\text { Spongeliomorpha cf. } \\
\text { sudolica } \\
\text { Fig. 26A, B }\end{array}$ & $\begin{array}{l}\text { Endichnial, horizontal, cylindrical bur- } \\
\text { rows } 10-20 \text { mm in diameter, showing Y- } \\
\text { and T-shape branching and subtle wall } \\
\text { ornamentation with short, crisscross- } \\
\text { ing, dull ridges oblique to burrow axis. } \\
\text { Differs from Spongeliomorpha sudolica } \\
\text { by the locally visible ornamentation in } \\
\text { the form of lanceolate ridges }\end{array}$ & $\begin{array}{l}\text { Fodinichnion } \\
\text { domichnion }\end{array}$ & Decapods & $\begin{array}{l}\text { Found as several speci- } \\
\text { mens of various segments } \\
\text { of burrow system in the ZMb }\end{array}$ & $\begin{array}{l}\text { Zaręczny (1878), } \\
\text { Marcinowski and } \\
\text { Wierzbowski } \\
\text { (1975), Gibert and } \\
\text { Robles (2005) }\end{array}$ \\
\hline $\begin{array}{c}\text { ?Taenidium baretti } \\
\text { Fig. 38A, B }\end{array}$ & $\begin{array}{l}\text { Straight to curved, indistinctly branched, } \\
\text { cylindrical, meniscate backfilled, horizon- } \\
\text { tal to subhorizontal burrows, } 16-30 \mathrm{~mm} \text { in } \\
\text { diameter, lined discontinuously with sedi- } \\
\text { ment enriched in organic particles and i- } \\
\text { regular to knobbly sand aggregates. Infill } \\
\text { menisci are hemispherical or deeply arcu- } \\
\text { ate, tightly packed, irregularly compart- } \\
\text { mentalized as heterogeneous trace } \\
\text { segments. These forms were classified as } \\
\text { Taenidium baretti by Leszczyński (2010), } \\
\text { but their local lining and signs of branching } \\
\text { impugn affiliation with Taenidium }\end{array}$ & Fodinichnion & Arthropods & $\begin{array}{l}\text { Found as several speci- } \\
\text { mens a few } \mathrm{cm} \text { long in } \\
\text { muddy sandstone of a he- } \\
\text { terolithic package in the } \\
\text { CFm at locality } 9\end{array}$ & $\begin{array}{l}\text { Keighley and } \\
\text { Pickerill (1994), } \\
\text { Bromley et al. } \\
\text { (1999), } \\
\text { Leszczyński } \\
(2010)\end{array}$ \\
\hline
\end{tabular}


Tab. 1 cont.

\begin{tabular}{|c|c|c|c|c|c|}
\hline $\begin{array}{l}\text { Ichnogenus/ichnospe } \\
\text { cies (with photo- } \\
\text { graph references) }\end{array}$ & Short description & Ethology & $\begin{array}{l}\text { Inferred } \\
\text { producer }\end{array}$ & Occurrence & Key references \\
\hline $\begin{array}{c}\text { ?Taenidium crassum } \\
\text { Fig. } 33 \mathrm{E}\end{array}$ & $\begin{array}{l}\text { Unbranched, straight or sinuous cylindri- } \\
\text { cal burrows, unlined or very thinly lined, } \\
\text { with an infill containing parabolic or chev- } \\
\text { ron-shaped sediment packets, generally } \\
\text { asymmetrical, with the parabola or chev- } \\
\text { ron apex offset relative the infill midline }\end{array}$ & Fodinichnion & Arthropods & $\begin{array}{l}\text { Found as several speci- } \\
\text { mens a few cm long in } \\
\text { muddy sandstone of a he- } \\
\text { terolithic package in the } \\
\text { CFm at locality } 9\end{array}$ & $\begin{array}{c}\text { Keighley and } \\
\text { Pickerill (1994), } \\
\text { Bromley et al. } \\
(1999)\end{array}$ \\
\hline $\begin{array}{l}\text { ? Taenidium isp. A } \\
\text { Fig. } 38 \mathrm{C}\end{array}$ & $\begin{array}{l}\text { Unbranched, straight or slightly curved } \\
\text { horizontal to oblique cylindrical bur- } \\
\text { rows, 4-5 mm in diameter and several } \\
\mathrm{cm} \text { long, with unlined wall and a } \\
\text { meniscate infill showing heterogeneous } \\
\text { packets of hemispherical menisci }\end{array}$ & Fodinichnion & Arthropods & $\begin{array}{l}\text { Found as a few specimens } \\
\text { in muddy sandstone of a } \\
\text { heterolithic package in the } \\
\text { CFm at locality } 9\end{array}$ & \\
\hline $\begin{array}{l}\text { ?Taenidium isp. B } \\
\text { Fig. 38D }\end{array}$ & $\begin{array}{l}\text { Bedding-oblique burrows, irregularly } \\
\text { lined, with a segmented heterogeneous } \\
\text { infill made of hemispherical menisci }\end{array}$ & Fodinichnion & Arthropods & $\begin{array}{l}\text { Found as one specimen in } \\
\text { muddy sandstone of a he- } \\
\text { terolithic package in the } \\
\text { CFm at locality } 9\end{array}$ & \\
\hline $\begin{array}{c}\text { Taxichnites ?wurmi } \\
\text { Fig. } 29 \text { B }\end{array}$ & $\begin{array}{l}\text { Bow-shaped burrow system, } 10 \mathrm{~mm} \text { wide } \\
\text { and } 35 \mathrm{~mm} \text { long, resembling a conifer } \\
\text { twig, showing two rows of tiny chevron } \\
\text { lanceolate ridges (furrows) } 2-5 \mathrm{~mm} \text { long } \\
\text { and } 1-2.6 \mathrm{~mm} \text { wide without marked axis }\end{array}$ & Fodinichnion & Arthropods & $\begin{array}{l}\text { Found as a single speci- } \\
\text { men on sandstone bed so- } \\
\text { le at locality } 6\end{array}$ & $\begin{array}{l}\text { Stepanek and } \\
\text { Geyer (1989), } \\
\text { Seilacher (2007) }\end{array}$ \\
\hline $\begin{array}{l}\text { ? Teichichnus isp. } \\
\text { Figs. } 16 \text { D and } 34\end{array}$ & $\begin{array}{l}\text { Long, straight, wall-like spreiten burrows } \\
\text { up to } 10 \mathrm{~cm} \text { long and } 3 \mathrm{~cm} \text { high, with con- } \\
\text { cave-up horizontal long axis and with } \\
\text { gently concave, vertically stacked gut- } \\
\text { ter-shaped horizontal spreite laminae } \\
\text { with slightly inclined basal part. These } \\
\text { burrows in vertical section can be con- } \\
\text { fused with Asterosoma }\end{array}$ & Fodinichnion & $\begin{array}{l}\text { Worm-like } \\
\text { animals }\end{array}$ & $\begin{array}{l}\text { Found as a mass occur- } \\
\text { rence in a vertical section } \\
\text { of heterolith package in the } \\
\text { CFm at locality } 9\end{array}$ & \\
\hline $\begin{array}{l}\text { Teredolites clavatus } \\
\text { Fig. 12B-F } \\
\text { Leszczyński } \\
\text { (2010: fig. 10E, F) }\end{array}$ & $\begin{array}{l}\text { Fine-sand casts of clavate full reliefs, 4-7 } \\
\mathrm{mm} \text { in diameter, in completely weathered } \\
\text { driftwood, perpendicular to the wood } \\
\text { grain. Casts deformed by wood compac- } \\
\text { tion, with a length-to-width ratio of <3 } \\
\end{array}$ & Domichnion & $\begin{array}{l}\text { Wood-bor- } \\
\text { ing bivalves }\end{array}$ & $\begin{array}{l}\text { Found as clusters in sev- } \\
\text { eral isolated driftwood frag- } \\
\text { ments and in wood lag in } \\
\text { the ZMb and in the CFm at } \\
\text { locality } 9\end{array}$ & $\begin{array}{l}\text { Leymerie (1842); } \\
\text { Kelly and Bromley } \\
\text { (1984), Savrda } \\
\text { and Smith (1996) }\end{array}$ \\
\hline $\begin{array}{l}\text { Teredolites cf. } \\
\text { longissimus } \\
\text { Fig. } 12 \mathrm{~A}\end{array}$ & $\begin{array}{l}\text { Fine-sand casts of cylindrical elongate } \\
\text { full reliefs, 4-8 mm in diameter, in com- } \\
\text { pletely weathered driftwood. }\end{array}$ & $\begin{array}{l}\text { Domichnion, } \\
\text { fodinichnion }\end{array}$ & $\begin{array}{l}\text { Wood-bor- } \\
\text { ing bivalves }\end{array}$ & $\begin{array}{l}\text { Solitary cluster of speci- } \\
\text { mens found in the ZMb at } \\
\text { locality } 8\end{array}$ & $\begin{array}{c}\text { Kelly and Bromley } \\
(1984), \text { Savrda } \\
\text { and Smith (1996) }\end{array}$ \\
\hline $\begin{array}{l}\text { Thalassinoides } \\
\text { paradoxicus } \\
\text { Fig. 23A-C }\end{array}$ & $\begin{array}{l}\text { Endichnial and hypichnial cylindrical hori- } \\
\text { zontal to vertical burrows, with small cy- } \\
\text { lindrical offshoots and rough to smooth } \\
\text { walls, irregularly branched (T-shaped } \\
\text { junctions more common than Y-shaped } \\
\text { bifurcations). Burrow diameter 5-35 mm, } \\
\text { occasionally enlarged at branching } \\
\text { points. Infill similar to the host sand }\end{array}$ & $\begin{array}{l}\text { Domichnion, } \\
\text { fodinichnion }\end{array}$ & $\begin{array}{l}\text { Decapod } \\
\text { crustaceans }\end{array}$ & $\begin{array}{l}\text { Portions of several systems } \\
\text { found in the completely bio- } \\
\text { turbated top part of upper- } \\
\text { most sandstone bed in the } \\
\text { ZMb at locality } 5 \text {. Many spe- } \\
\text { cimens found in the CFm at } \\
\text { localities } 5 \text { and } 9\end{array}$ & $\begin{array}{l}\text { Fürsich (1973), } \\
\text { Howard and Frey } \\
(1984)\end{array}$ \\
\hline $\begin{array}{l}\text { Thalassinoides } \\
\text { suevicus } \\
\text { Figs. 23D-H, } \\
\text { 31G-J, 37C }\end{array}$ & $\begin{array}{l}\text { Cylindrical, unlined, smooth-walled hor- } \\
\text { izontal burrows, 10-30 mm in diameter, } \\
\text { commonly branched and slightly enlar- } \\
\text { ged at branching points, with Y-shaped } \\
\text { bifurcations more common than } \\
\text { T-shaped junctions. Burrow infill not al- } \\
\text { ways similar to the host sand }\end{array}$ & $\begin{array}{l}\text { Domichnion, } \\
\text { fodinichnion }\end{array}$ & $\begin{array}{l}\text { Decapod } \\
\text { crustaceans }\end{array}$ & $\begin{array}{l}\text { Found as several maze } \\
\text { systems and burrow seg- } \\
\text { ments on top of sandstone } \\
\text { beds in the ZMb and as } \\
\text { many specimens in the } \\
\text { CFm at localities } 5 \text { and } 9\end{array}$ & $\begin{array}{c}\text { Frey et al. (1984), } \\
\text { Frey and Howard } \\
(1990), \\
\text { Schlirf (2000) }\end{array}$ \\
\hline $\begin{array}{l}\text { Thalassinoides isp. } \\
\text { Figs. 24A, B, 25A, } \\
27 \mathrm{~A}, \mathrm{~B}\end{array}$ & $\begin{array}{l}\text { Cylindrical, unlined, horizontally oriented, } \\
\text { rarely branched full relief showing } \\
\text { Y-shaped branching; } 10 \mathrm{~mm} \text { in diameter }\end{array}$ & $\begin{array}{l}\text { Domichnion, } \\
\text { fodinichnion }\end{array}$ & $\begin{array}{l}\text { Decapod } \\
\text { crustaceans }\end{array}$ & $\begin{array}{l}\text { Noted in many specimens in } \\
\text { sandstone of ZM. In Warto- } \\
\text { wice they occur accompa- } \\
\text { nying Ophiomorha nodosa } \\
\end{array}$ & $\begin{array}{l}\text { Frey et al. (1984), } \\
\text { Frey and Howard } \\
(1990)\end{array}$ \\
\hline $\begin{array}{l}\text { aff. Thalassinoides } \\
\text { isp. } \\
\text { Figs. } 16 \mathrm{D}, 24 \mathrm{C} \\
\text { and } 32 \mathrm{~A}, \mathrm{~B}\end{array}$ & $\begin{array}{l}\text { Endichnial, horizontal, irregular cylindri- } \\
\text { cal burrows, 5-20 mm in diameter, show- } \\
\text { ing indistinct T-shape and Y-shape } \\
\text { branching, with irregular walls and off- } \\
\text { shoots mainly thinner than the main bur- } \\
\text { row }\end{array}$ & $\begin{array}{l}\text { Fodinich- } \\
\text { nion, } \\
\text { domichnion }\end{array}$ & $\begin{array}{l}\text { Decapod } \\
\text { crusta- } \\
\text { ceans? }\end{array}$ & $\begin{array}{l}\text { Found as casts of short bur- } \\
\text { row segments on sandstone } \\
\text { bed sole in ZMb at locality } 1 \mathrm{~B} \\
\text { and as full casts and vertical } \\
\text { section in a coal seam in } \\
\mathrm{NMb}(\mathrm{CFm}) \text { at locality } 9\end{array}$ & $\begin{array}{l}\text { Frey et al. (1984), } \\
\text { Frey and Howard } \\
\qquad(1990)\end{array}$ \\
\hline $\begin{array}{l}\text { Treptichnus aff. } \\
\text { bifurcatus } \\
\text { Fig. 29C, D }\end{array}$ & $\begin{array}{l}\text { Horizontal, slightly curved row of short } \\
\text { burrow segments arranged at the angle } \\
\text { to the row, projecting mainly to one side; } \\
\text { burrow diameter } 2-3 \mathrm{~mm}\end{array}$ & Fodinichnion & $\begin{array}{l}\text { Vermiform } \\
\text { animals }\end{array}$ & $\begin{array}{l}\text { Recorded in several speci- } \\
\text { mens on lower surface of san- } \\
\text { dstone bed in ZM, in Skała. } \\
\text { From proper T. bifurcatus dif- } \\
\text { fers in the mainly shingled ar- } \\
\text { rangement of segments }\end{array}$ & $\begin{array}{l}\text { Schlirf }(2000) ; \\
\text { Rindsberg and } \\
\text { Kopaska- } \\
\text { Merkel (2005) }\end{array}$ \\
\hline \multicolumn{6}{|c|}{ Taxonomically undetermined trace fossils } \\
\hline $\begin{array}{c}\text { Pockmarked relief } \\
\text { Fig. 30B }\end{array}$ & $\begin{array}{l}\text { Irregular patches of curly sand lumps, } \\
\sim 4 \text { mm in size, and jaggy narrow crests } \\
\text { separated by short winding furrows }\end{array}$ & Pascichnia & $\begin{array}{l}\text { ?Fish or } \\
\text { arthropods }\end{array}$ & $\begin{array}{l}\text { Found on sharp bounding } \\
\text { surfaces of sandstone beds } \\
\text { in the ZMb at locality } 5\end{array}$ & \\
\hline $\begin{array}{l}\text { Curly lumps relief } \\
\text { Fig. 30C }\end{array}$ & $\begin{array}{l}\text { Irregular to lobate, elongate patches of } \\
\text { fuzzy and occasionally coil-shaped } \\
\text { sand lumps } \sim 2 \mathrm{~mm} \text { in size }\end{array}$ & Fodinichnion & $\begin{array}{l}\text { ?Fish or } \\
\text { arthropods }\end{array}$ & $\begin{array}{l}\text { Found on sharp bed-boun- } \\
\text { ding surfaces in sandsto- } \\
\text { nes of the ZMb at locality } 8 \\
\end{array}$ & \\
\hline $\begin{array}{l}\text { Irregular projections } \\
\text { Fig. 30D }\end{array}$ & $\begin{array}{l}\text { Hypichnial patches of irregularly sha- } \\
\text { ped and sized projections arranged } \\
\text { chaotically. Some projections have a } \\
\text { fish-shape outline }\end{array}$ & Repichnia & $\begin{array}{l}\text { ?Fish, } \\
\text { arthropods }\end{array}$ & $\begin{array}{l}\text { Found on sandstone bed } \\
\text { sole in the ZMb at locality } 9\end{array}$ & \\
\hline
\end{tabular}


Tab. 1 cont.

\begin{tabular}{|c|c|c|c|c|c|}
\hline $\begin{array}{c}\begin{array}{c}\text { Ichnogenus/ichnospe } \\
\text { cies } \\
\text { (with photograph ref- } \\
\text { erences) }\end{array} \\
\end{array}$ & Short description & Ethology & $\begin{array}{l}\text { Inferred } \\
\text { producer }\end{array}$ & Occurrence & Key references \\
\hline $\begin{array}{c}\text { Cane-patterned relief } \\
\text { Fig. } 30 \mathrm{~A}\end{array}$ & $\begin{array}{l}\text { Epichnial panicles and tighter bunches } \\
\text { of cane-shaped furrows, } 1 \mathrm{~mm} \text { wide and } \\
\text { up to } 15 \mathrm{~mm} \text { long, locally overlapped } \\
\text { and surrounded by irregular aureole of } \\
\text { lumpish sediment }\end{array}$ & Pascichnia & Annelids & $\begin{array}{l}\text { Found on a rippled top of a } \\
\text { sandstone bed in the ZMb } \\
\text { at locality } 5\end{array}$ & \\
\hline $\begin{array}{l}\text { Lamellate structures } \\
\text { Fig. 32D }\end{array}$ & $\begin{array}{l}\text { System of clustered burrows, in parts ver- } \\
\text { tically and obliquely laterally lamellate. } \\
\text { Clustering resembles Phycodes. Uncer- } \\
\text { tain association with Thallasinoides-like } \\
\text { burrows }\end{array}$ & Fodinichnion & Unknown & $\begin{array}{l}\text { Found in a coal seam in the } \\
\text { CFm at locality } 9\end{array}$ & \\
\hline $\begin{array}{l}\text { Lumpy leveed } \\
\text { groove } \\
\text { Fig. } 27 \mathrm{~F}\end{array}$ & $\begin{array}{l}\text { Short epirelief consisting of a central } \\
\text { groove, } 2 \mathrm{~mm} \text { wide and up to } 10 \mathrm{~mm} \\
\text { long, flanked on either side by a lumpy } \\
\text { levee up to } 5 \mathrm{~mm} \text { wide. Resemble a trail } \\
\text { of Cerithidea californica }\end{array}$ & Cubichnion & $\begin{array}{l}\text { Gastropods } \\
\text { or arthro- } \\
\text { pods }\end{array}$ & $\begin{array}{l}\text { Found as many specimens } \\
\text { on tops of sandstone beds } \\
\text { in the } \mathrm{ZMb} \text { at locality } 5\end{array}$ & $\begin{array}{c}\text { Knox and Miller } \\
(1985)\end{array}$ \\
\hline $\begin{array}{c}\text { Branched 3D swirls } \\
\text { Fig. 39A }\end{array}$ & $\begin{array}{l}\text { Endichnial system of clustered, curved, } \\
\text { densely branched, vertical and lateral } \\
\text { cylindrical burrows, } 2.2 \mathrm{~mm} \text { in diameter }\end{array}$ & Fodinichnion & $\begin{array}{l}\text { ?Arthropods } \\
\text { or ?annelids }\end{array}$ & $\begin{array}{l}\text { Found in a small claystone } \\
\text { block detached from an out- } \\
\text { crop of the CFm at locality } 12\end{array}$ & \\
\hline $\begin{array}{l}\text { 3D swirls } \\
\text { Fig. 39B }\end{array}$ & $\begin{array}{l}\text { Endichnial system of curved, vertical } \\
\text { and lateral cylindrical burrows, } 2 \mathrm{~mm} \text { in } \\
\text { diameter, swirled and unbranched, with } \\
\text { local mud mantle up to } 1.5 \mathrm{~mm} \text { thick }\end{array}$ & Fodinichnion & ?Annelids & $\begin{array}{l}\text { Found in a small block of } \\
\text { muddy sandstone deta- } \\
\text { ched from an outcrop of the } \\
\text { CFm at locality } 12\end{array}$ & \\
\hline $\begin{array}{l}\text { Branched lump } \\
\text { Fig. 39C }\end{array}$ & $\begin{array}{l}\text { An irregular chamber (lump), up to } 2 \mathrm{~cm} \\
\text { in cross-section, preserved in full relief, } \\
\text { with horizontally radiating curved tun- } \\
\text { nels } 1.5 \mathrm{~mm} \text { in diameter. Infill slightly } \\
\text { coarser-grained than the host mud }\end{array}$ & ?Calichnion & ?Arthropods & $\begin{array}{l}\text { Found as a solitary speci- } \\
\text { men in a small block loose } \\
\text { of sandy mudstone deta- } \\
\text { ched from an outcrop of the } \\
\text { CFm at locality } 12\end{array}$ & \\
\hline
\end{tabular}

Localities as in Table 1 and lithostratigraphic acronyms as in the text
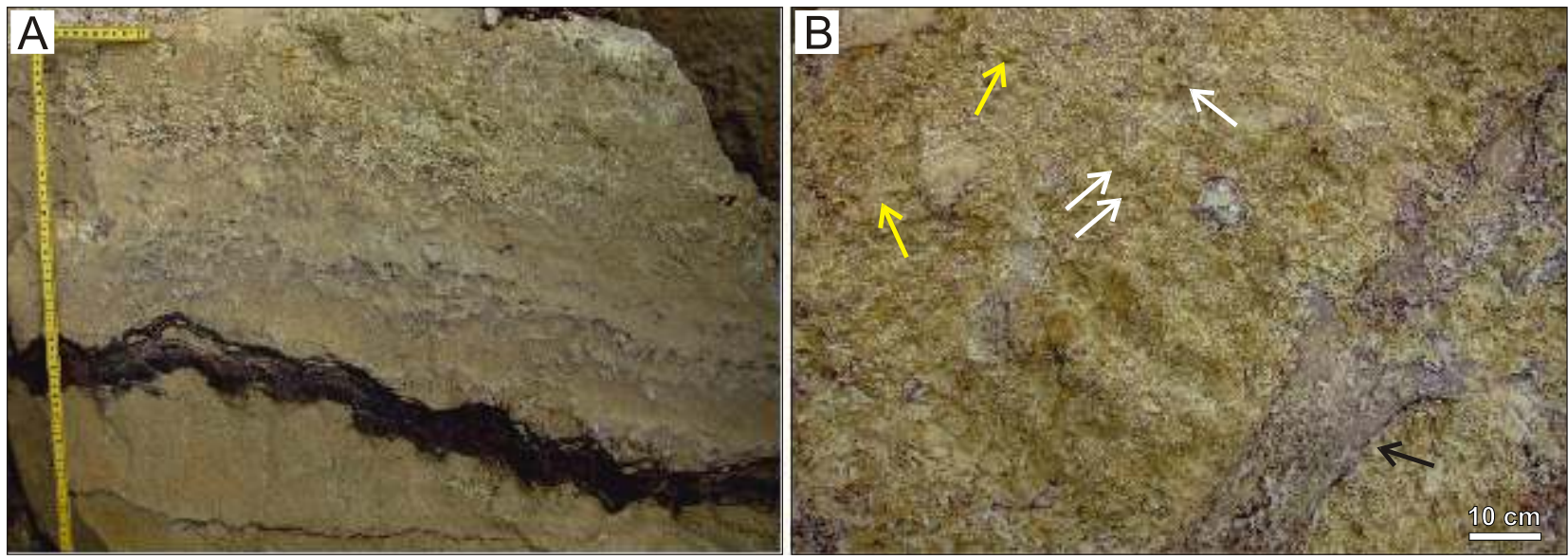

Fig. 13. Bioturbation features in the top part of erkowice Member

A - ichnofabric and upwards increasing bioturbation intensity in a sandstone bed, locality 5 (Wartowice), photography August 2011; B - highly bioturbated top of sandstone bed, stained with $\mathrm{Fe}$ - oxide/hydroxide, showing rare tunnels (yellow arrow) and shafts (white arrow) of an undetermined cylindrical trace fossil and a suspected imprint of a tree trunk (black arrow), same locality and photography date

black mudstones and where a couple of coarsening-upwards packages of mudstones passing into heterolithic deposits culminate in fine-grained sandstones.

The thicker sandstones in the CFm are friable, fine- to coarse-grained, with a local admixture of granules and small pebbles. Some are massive, in at least some cases due to pervasive bioturbation, whereas others show parallel, horizontal to gently inclined stratification, through cross-stratification, ripple cross-lamination and rarely planar cross-stratification (Fig. 15D). Bioturbation structures occur particularly at localities 5 and 9. At this latter locality, there are also imprints and casts of scattered mollusc shells, some in a life position, as well as imprints of shell lags (see Leszczyński, 2010). At locality 5, several brownish-red to brown ferruginized horizons and ferricrete layers occur (Fig. 7). In the central sector of the quarry at locality 9 , the lower part of the CFm, up to the upper coal bed, is tectonically duplexed over a distance of $\sim 13$ m (Fig. 6B; Leszczyński, 2010).

\section{TRACE FOSSILS AND BIOTURBATION PATTERN}

Bioturbation structures occur erratically in the studied deposits. Their distribution varies both vertically and laterally, depending mainly upon lithofacies. Vertical changes occur at a 

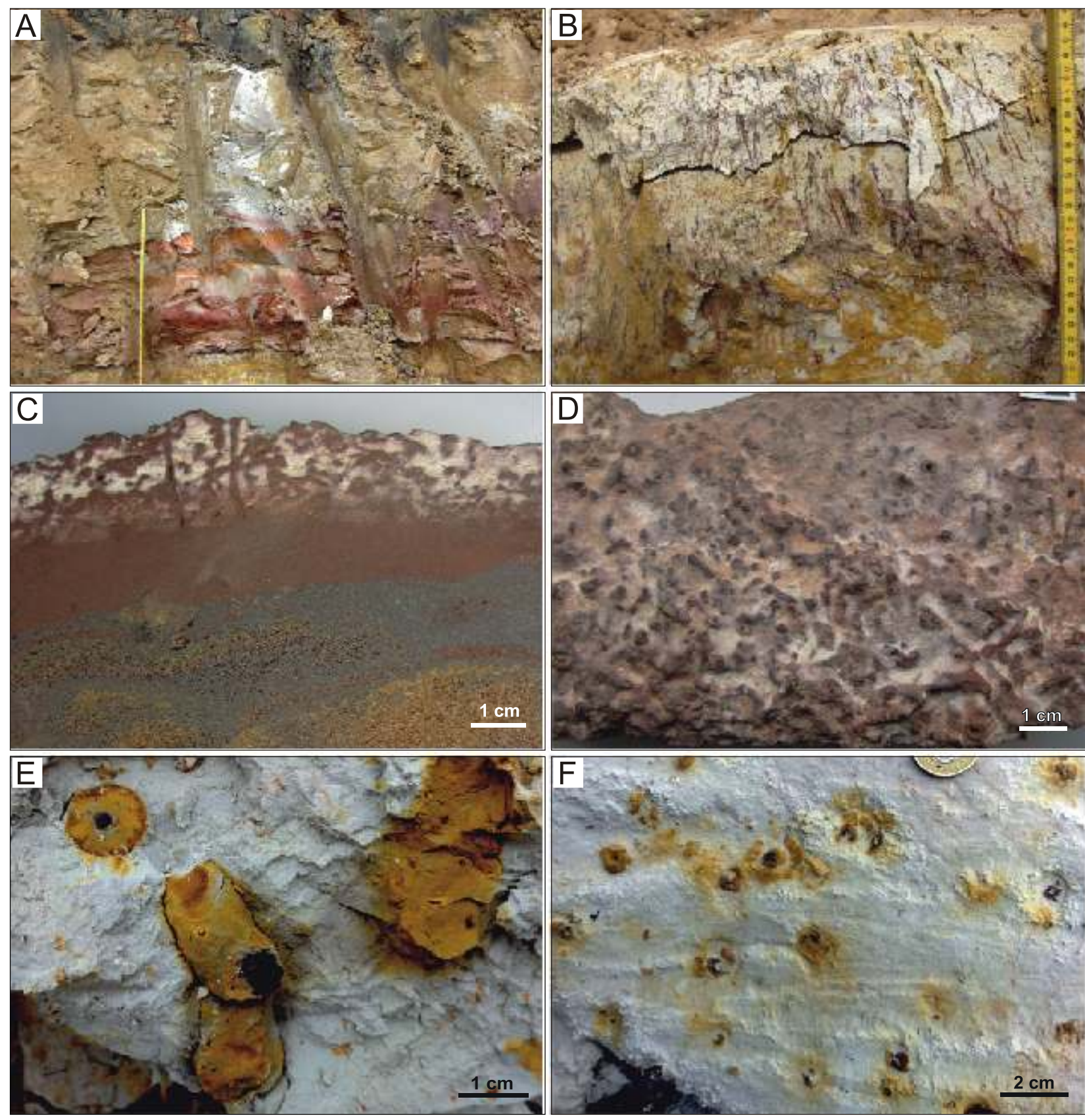

Fig. 14. Sedimentary features of the boundary interval between the erkowice Member and Czerna Formation at locality 5 (Wartowice)

A - the lowest part of the Czerna Formation ( 2 $\mathrm{m}$ ) with a plant-rooted sandstone overlain by claystone and thin layer of allochthonous coal, photography May 2013; B - close-up view of the plant-rooted sandstone underlain by a whitish- grey clayey siltstone with goethite rhizocretions; C, D - ferruginized plant-root traces seen in vertical section of a ferricrete horizon and on bedding surface, respectively, photography August 2012; E, F - goethitic rhizocretions (orange) in clayey siltstone seen on bedding-oblique and bedding-parallel surface, respectively, photography May 2010

scale of both individual beds and lithostratigraphic units, with the ZMb deposits showing entirely different trace-fossil assemblages than the CFm deposits (Table 2; Figs. 12, 14-39).

In the ZMb deposits, except for the outcrops at locality 11 non-bioturbated sandstones dominate. This pertains particularly to localities 1,2 and 9, where bioturbation structures occur as isolated endichnial burrows and only sporadic burrows on bedding surfaces (observed in loose sandstone blocks). Burrows are more common in the outcrops at localities 3, 4 and $6-8$, and are most extensively distributed at locality 11 , where the outcrop section $>5 \mathrm{~m}$ thick (Fig. 17) shows $\mathrm{BI}=3$ to 6 and where intense bioturbation occurs as irregular patches. One or more intervals with bioturbation structures were recognized in sandstones at localities 5, 10 and 12 (Fig. 7). These intervals are $0.3-2.5 \mathrm{~m}$ thick, with sharp tops, completely bioturbated topmost part $(5-30 \mathrm{~cm})$ and a downwards-decreasing concentration of burrows (Fig. 10A, C-E). Structures reminiscent of plant-root casts have been found in such an interval $\sim 4 \mathrm{~m}$ below the ZMb top at locality 5 , where evident plant-root structures occur at the ZMb top. 

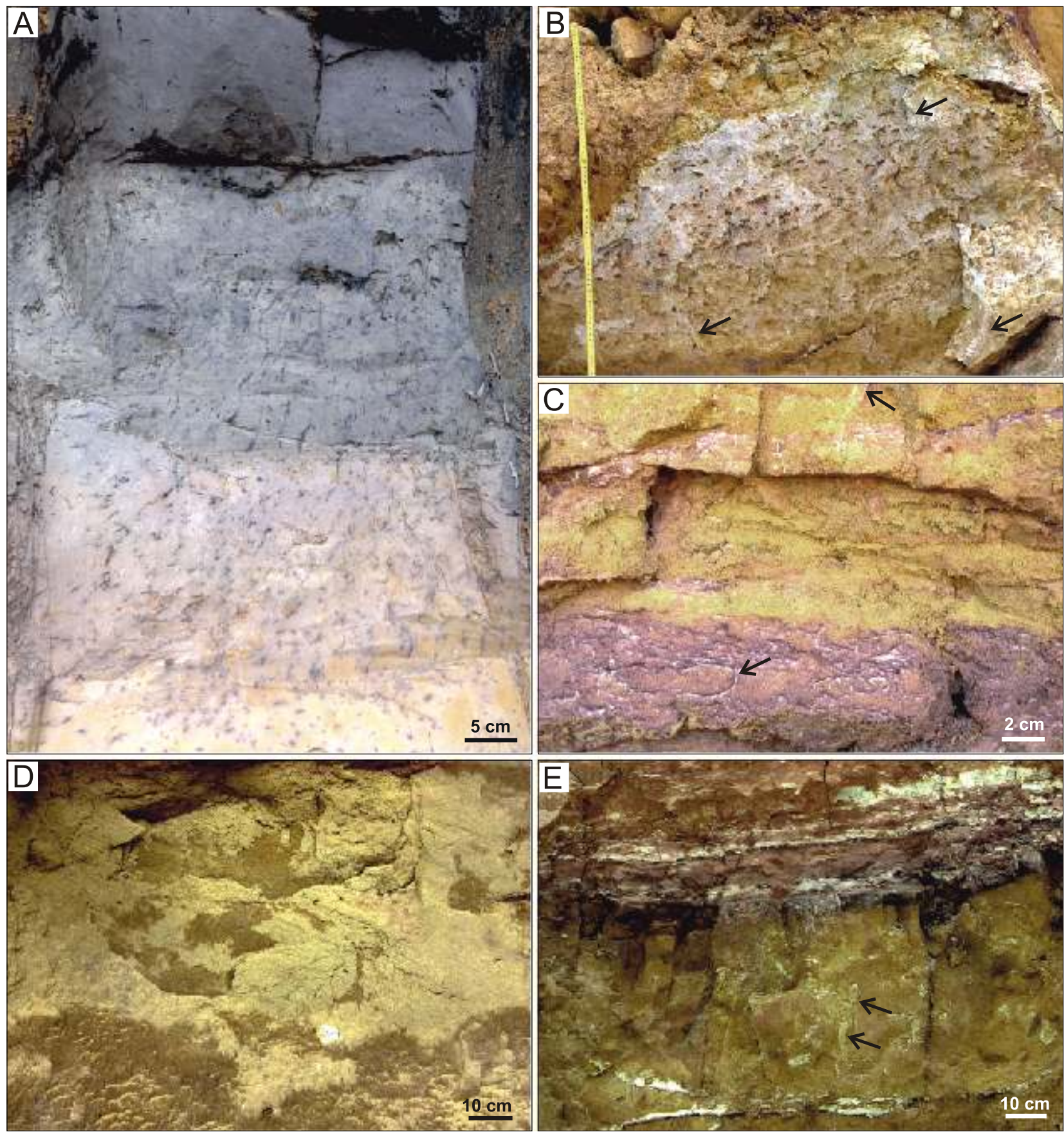

Fig. 15. Sedimentary features of the Czerna Formation at locality 5 (Wartowice)

A - clayey seatearth (bog vertisol) with dark thread-like plant-root traces and thin coal bed at the top, photograph May 2013; B, C, E three successive intervals of deposits above coal bed, showing sandstone with burrows overprinted by dispersed Ophiomorpha (arrows), stronger bioturbated ferruginized top part of sandstone and an overlying package of mudstone-dominated, variegated (ferruginized) heterolithic deposits, photographs October 2010 (B, C) and August 2011 (E); D - large-scale planar cross-stratified sandstone lacking bioturbation structures, photograph August 2011

Due to the nearly horizontal attitude of beds, their considerable thickness and outcrops in quarries, the bioturbation structures are accessible for investigation mainly on fracture surfaces transverse to bedding, whereas an access to bedding surfaces was limited to blocks derived from the quarry wall. These conditions hampered particularly the taxonomic identification of horizontally spread ichnia and their locating in the outcrop section. In total, 21 ichnogenera were determined in the
ZMb (Table 2 and Figs. 12A-E, 14, 17-29). Most of them were determined in open nomenclature, with five forms distinguished as taxonomically undetermined (Fig. 30).

Ophiomorpha, particularly $O$. nodosa, is the most common trace fossil in the sandstones of the $\mathrm{ZMb}$. Much less frequent is Thalassinoides (mainly $T$. suevicus, rarely $T$. paradoxicus) and intermediate forms. Other ichnotaxa occur either as solitary forms or as a few specimens, or in single beds only. They in- 

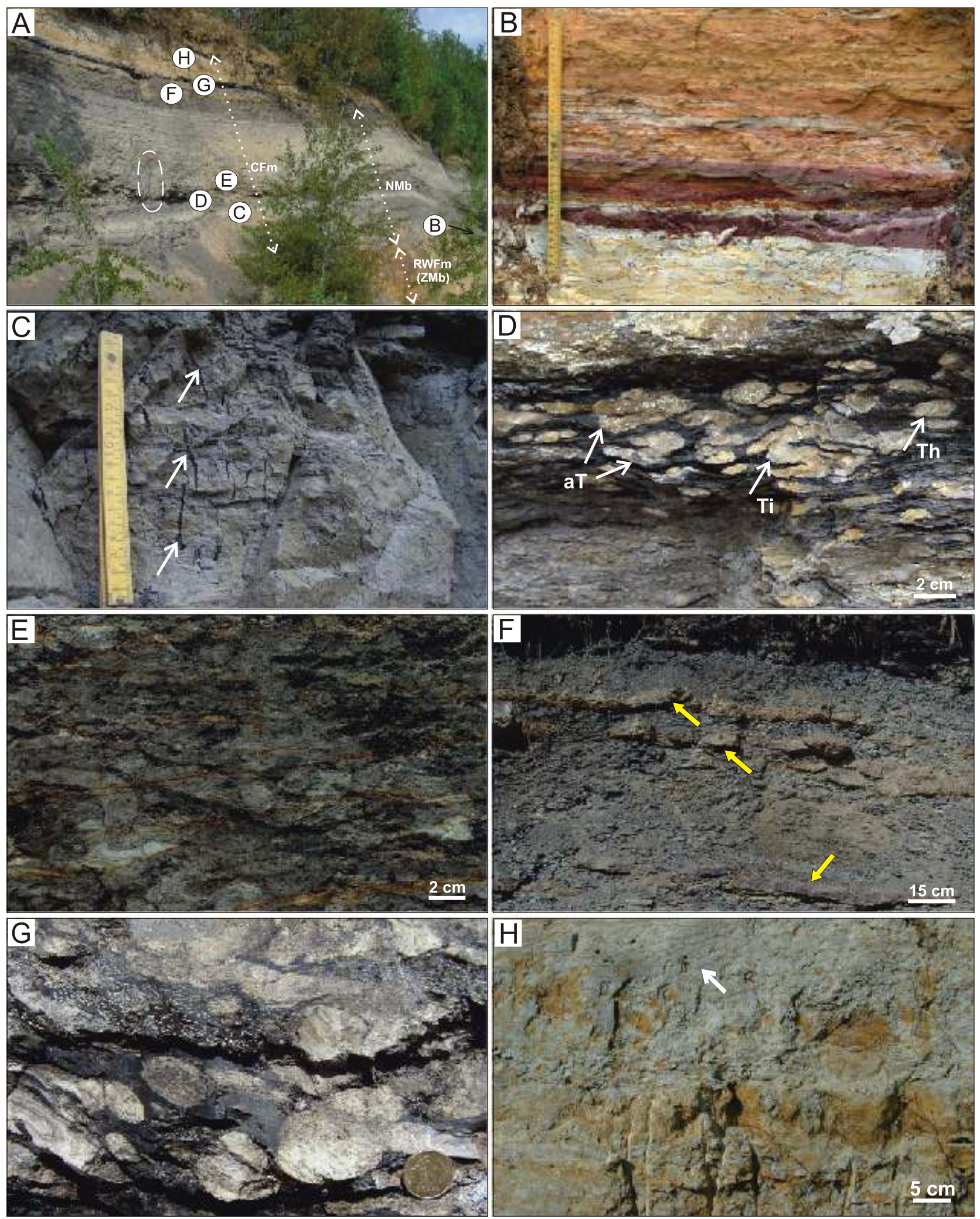

Fig. 16. Sedimentary features of the lowest part of the Czerna Formation at locality 9 (Rakowice Małe)

A - exposure of the Nowogrodziec Member (NMb) of the Czerna Formation (CFm) and the underlying part of the erkowice Member (ZMb) of the Rakowice Wielke Formation (RFm), photographed in May 2008, with letters B-H indicating location of close-up photograph and the walking stick (encircled) $1 \mathrm{~m}$ long; B - package of claystones and mudstones without distinct bioturbation structures, resting directly on sandstone of the erkowice Member; C - histosol with plant-root traces (arrows) beneath the lower coal bed, photography July 2007; D - the upper coal bed rich in sand-made trace fossils (light colour spots) including ? Teichichnus isp. (Ti), Thalassinoides isp. (Th) and aff. Thalassionoides isp. (aT), photography July 2007; E - sandstone-dominated heterolithic package interval almost completely disturbed by burrows, photography August 2009; F - the uppermost part of the Nowogrodziec Member, with dark grey to dark green mudstones bearing thin sideritized layers (arrows), photography May 2008; G - ichnofabric of sapropelic mudstone passing upwards into sandstone at the top of the Nowogrodziec Member (the light coloured spots are burrows), the coin (scale) is $1.5 \mathrm{~cm}$, photography May 2008; $\mathbf{H}$ - highly bioturbated sandstone with recognizable faint relics of planar cross-stratification (arrow), photography May 2008 

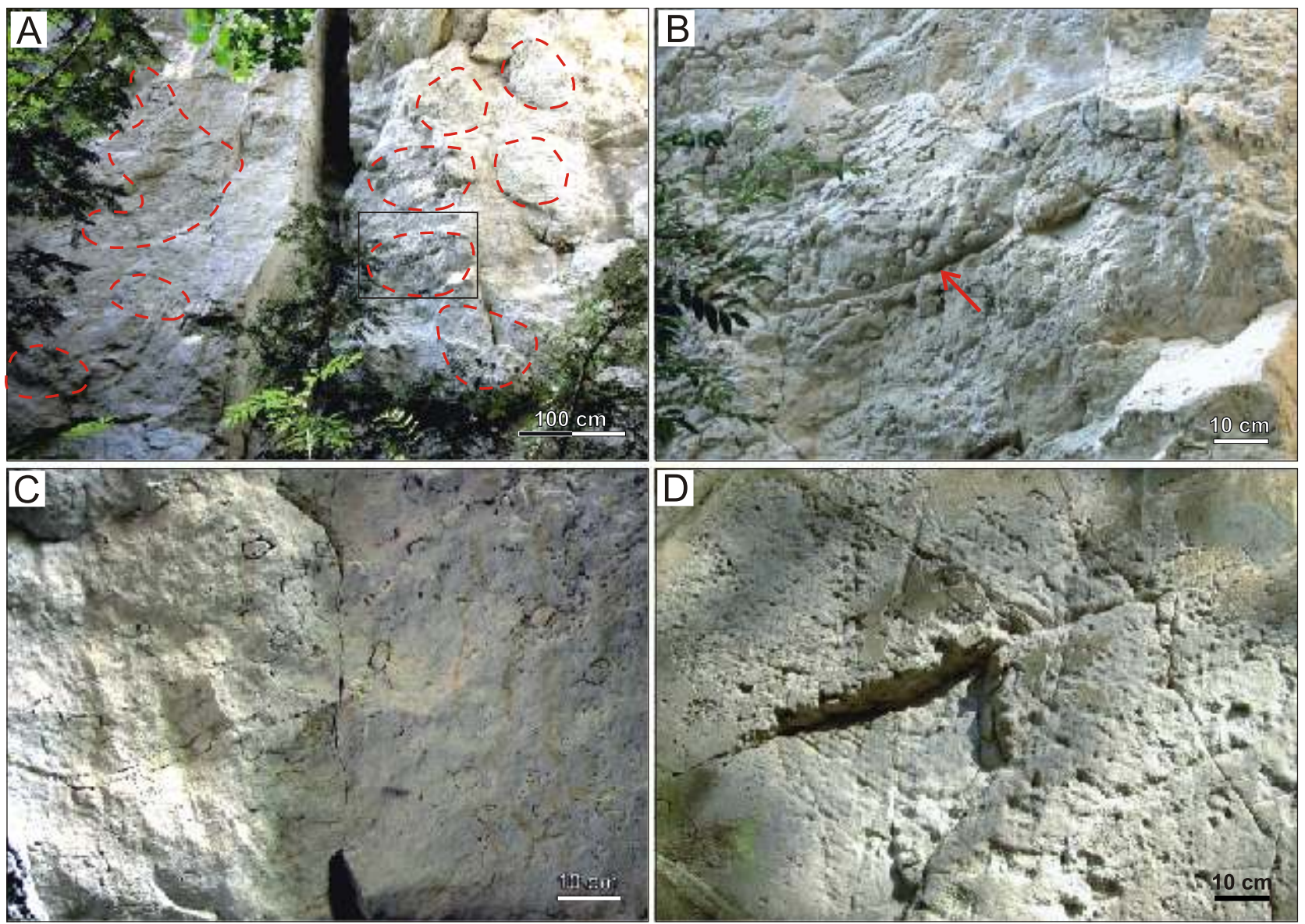

Fig. 17. Relationship between lithofacies and vertical distribution of burrows (mainly Ophiomorpha nodosa) in the most bioturbated sandstones of the erkowice Member at locality 11 (Milików)

A - outcrop section showing bioturbation $(\mathrm{BI}=3$ to 6$)$ in the entire thickness, with patchily aggregated burrows $(\mathrm{BI}=4$ to 6 , outlined in red) and the black rectangle indicating close-up in $\mathrm{B} ; \mathbf{B}$ - closer-up detail of the exposure, showing cross-sections of $O$. nodosa tunnels (diameter up to $4 \mathrm{~cm}$ ) and a relic trace of bed-bounding surface (arrow); C - portion of exposed section completely homogenized by bioturbation, dominated by $O$. nodosa tunnels up to $3 \mathrm{~cm}$ in diameter; $\mathbf{D}$ - exposure detail showing relationship between sandstone cross-stratification and its bioturbation

Fig. 18. Occurrence of Ophiomorpha nodosa in the sandstones of erkowice Member

A - tunnel-fill showing smooth-walled core mantled with tightly distributed rounded pellets (weathered out), the coin (scale) is $1.5 \mathrm{~cm}$, locality 4 ( eliszów), photography October 2010; B - shaft of solitary minute O. nodosa (arrow), locality 12 (Osiecznica), photography April, 2013; C - shaft mould showing pits after pellets arranged in rows transverse to burrow elongation, as in $O$. annulata (arrow), locality 5 (Wartowice), photography August 2011; D - steeply inclined burrow showing dispersed sandy pellets and remnants of earlier shaft to the right (arrow), same locality, photography August 2011; E - axial section of tunnel-fill with a sawtooth outline of pellets (arrow), some with a sandy core, same locality, photography May 2013; F - cluster of burrows with highly irregular linings and locally conical pellets, same locality, photography August 2011; G - segment of tunnel-fill with chaotic and locally pelleted lining (arrow), same locality, photography October 2010; $\mathbf{H}$ - tunnel-fill lined with loosely distributed muddy sand pellets on one side and an irregular wall on the other side, same locality, photography August $2011 ; \mathbf{I}$ - axial section of tunnel-fill showing an Fe-stained outline of pelleted lining with conical mud pellets (arrow), same locality, photography August 2011; J - longitudinal section of tunnel-fill marked by an Fe-stained outline (arrow), and faint meniscation, same locality, photography August $2011 ; \mathrm{K}$ - tunnel mould showing pellets arranged in rows transverse to burrow elongation (arrow), as in $O$. annulata, same locality, photography October 2011; L - meniscate tunnel-fill thinly lined with loosely distributed pellets (arrow), locality 8 ( erkowice), photography August 2011; M - outer surface of tunnel showing irregular, non-pelleted to pelleted lining with pellets formed as aggregates of smaller pellets (arrow), locality 5 (Wartowice), photography July 2013; $\mathbf{N}$ - mould of tunnel wall (arrow) and a segment of smooth-walled tunnel-fill to the right, same locality, photography October 2010 

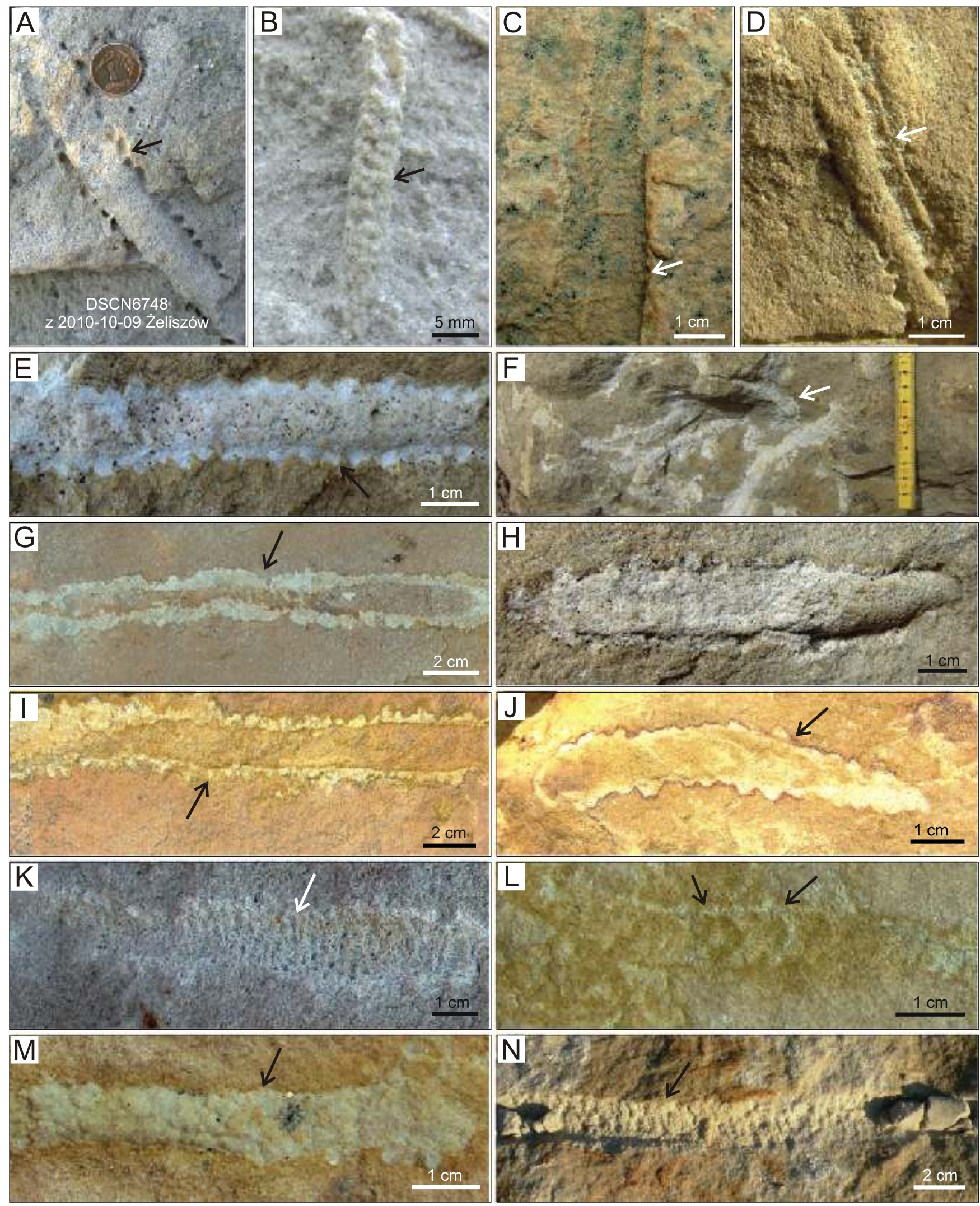

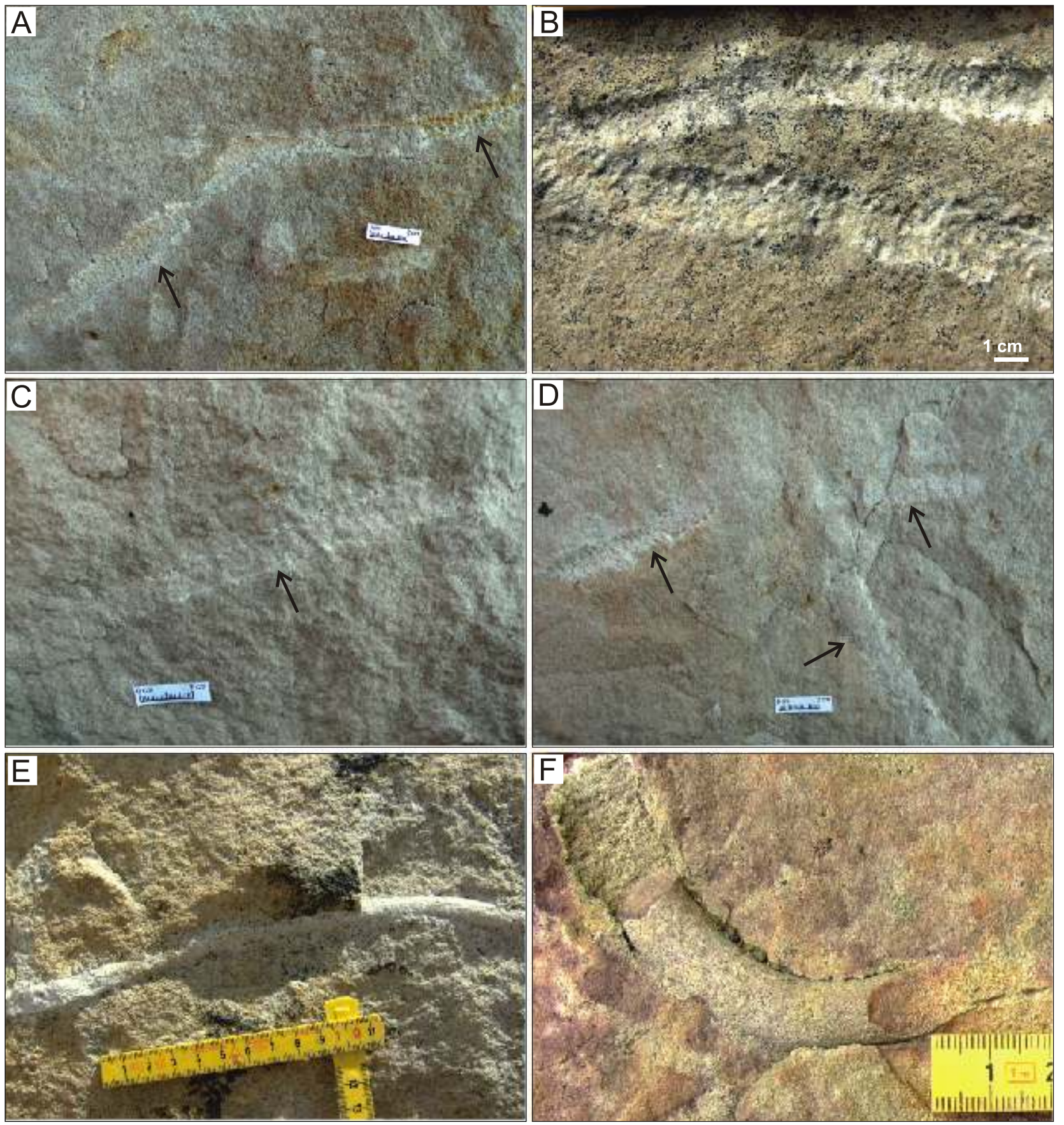

Fig. 19. Variable distinctiveness and lining style of Ophiomorpha nodosa in sandstones of the erkowice Member

A - specimen with its arrowed left-hand segment showing elongate pellets arranged transversely to burrow axis and the arrowed right-hand segment showing chaotically arranged larger, round pellets; B - two-branch specimen showing variable arrangement of pellets; $\mathbf{C}$ - light-coloured specimen (arrowed) with a faint and similarly pale lining, showing small, poorly visible and irregularly distributed pellets; D - specimens (arrowed) with a slightly lighter colour than that of the host sandstone and with a pitted imprint; E - specimen with a lining varying from distinct and pelleted to absent; $\mathbf{F}$ - specimen showing a faint lining with pellets solely as the lining mould. Locality 5 (Wartowice), photographs A, C and D from October 2010 and photographs B, E and F from August 2011

clude: Asterosoma isp., Aulichnites parkerensis, Biformites insolitus, ?Curvolithus isp. ?Cylindrichnus isp., ?Diplocraterion isp., Helminthopsis abeli, ?Lockeia isp., aff. Macaronichnus segregatis, ?Nereites irregularis, Ophioichnus isp., Palaeophycus sulcatus, Planolites beverleyensis, aff. Scolicia strozzii, ?Sinusichnus isp., Spongeliomorpha cf. sudolica, Taxichnites ?wurmi, Teredolites clavatus, T. cf. Iongissimus, Treptichnus aff. bifurcatus, plant-root structures and four undetermined ichnofossils. All rarely encountered ichnotaxa are mainly horizontally spreaded forms found on sandstone bedding surfaces.

The highest number of ichnotaxa have been found at localities 5, 6 and 8. In other outcrops, only one or a few forms have been found. Ophiomorpha is the only ichnofossil observed in all outcrops, although in some it was encountered as a single 

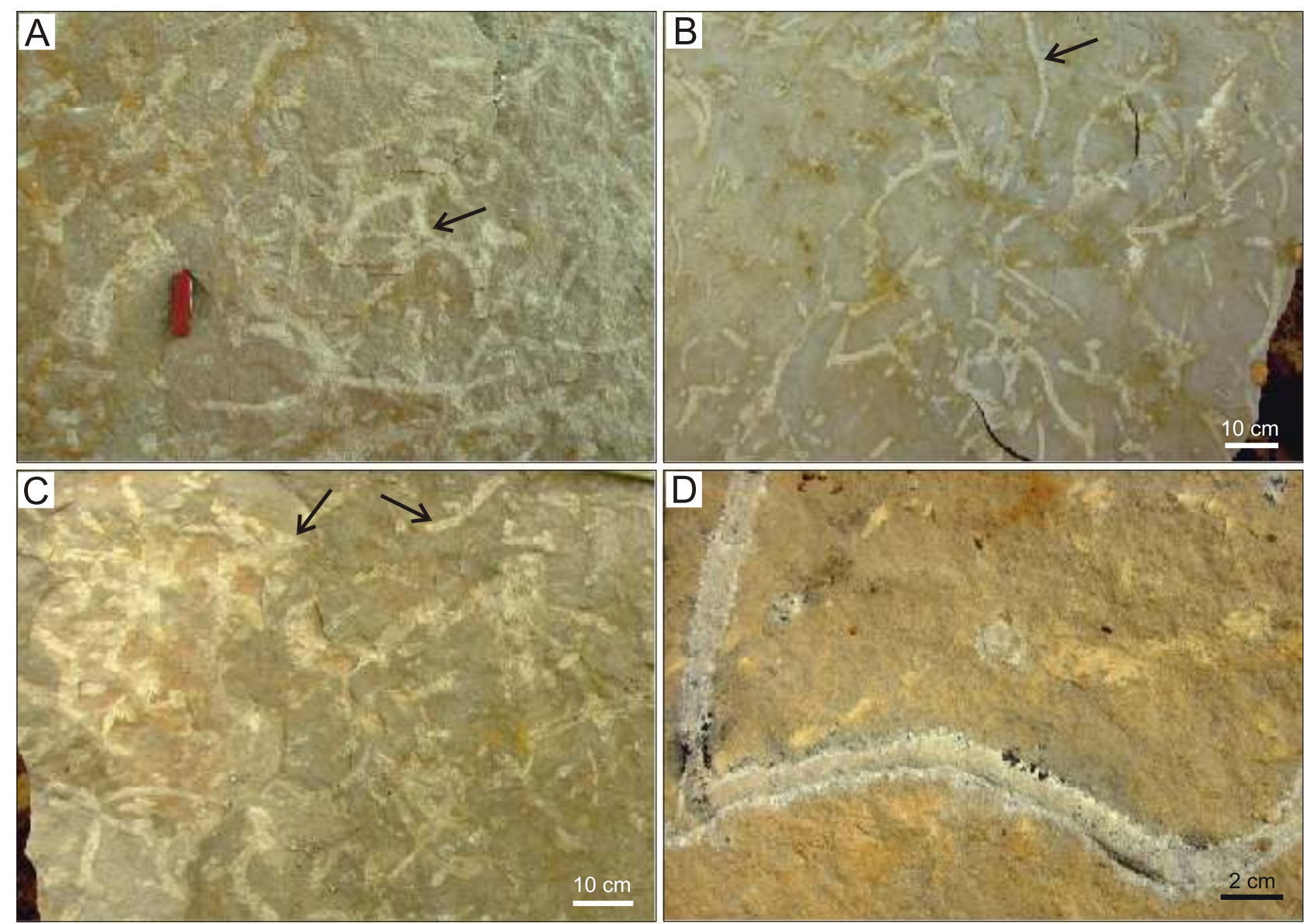

Fig. 20. Ophiomorpha nodosa on sandstone bedding surfaces in the erkowice Member at locality 5 (Wartowice)

Most distinct specimens are indicated by arrows. Note variation in the burrow size and density, lining type, course, style of branching and pattern of mutual criss-crossing; photography A from October 2010, photographs B and C from August 2011, and photography D from July 2013
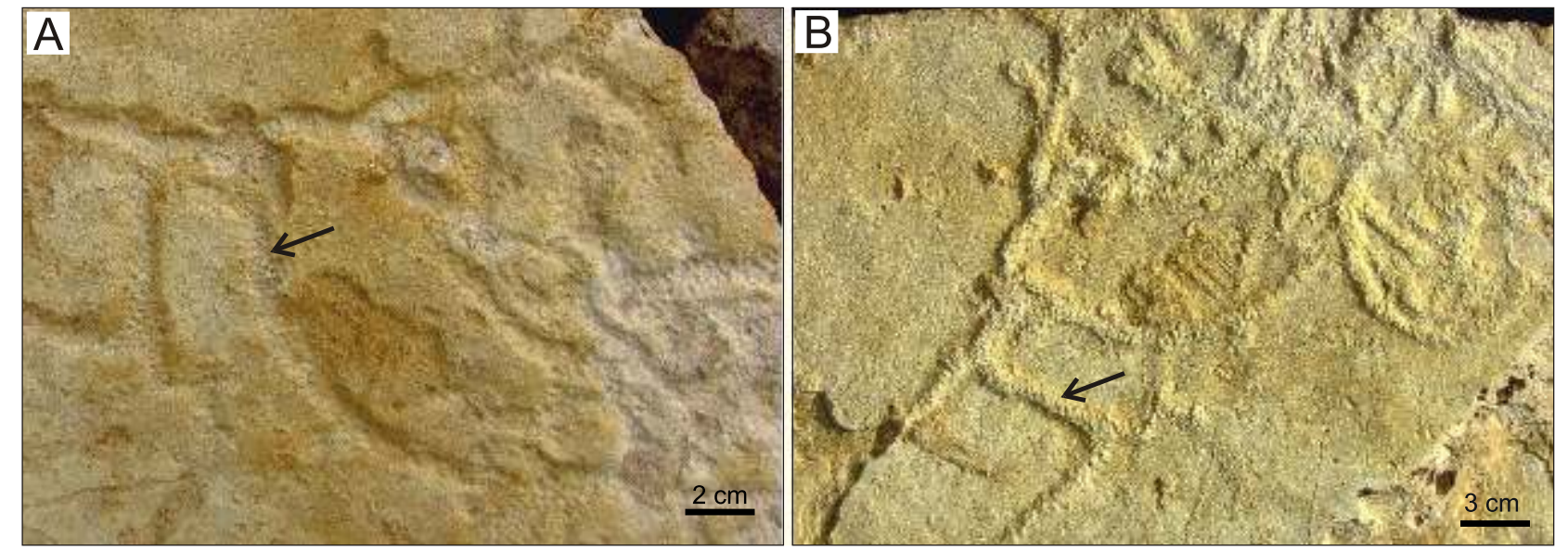

Fig. 21. Ophiomorpha ? nodosa on sandstone bedding surfaces in the erkowice Member at locality 5 (Wartowice)

Most distinct specimens are indicated by arrows; note that the pellets in $B$ tend to be arranged in pairs; photographs taken in August 2011 

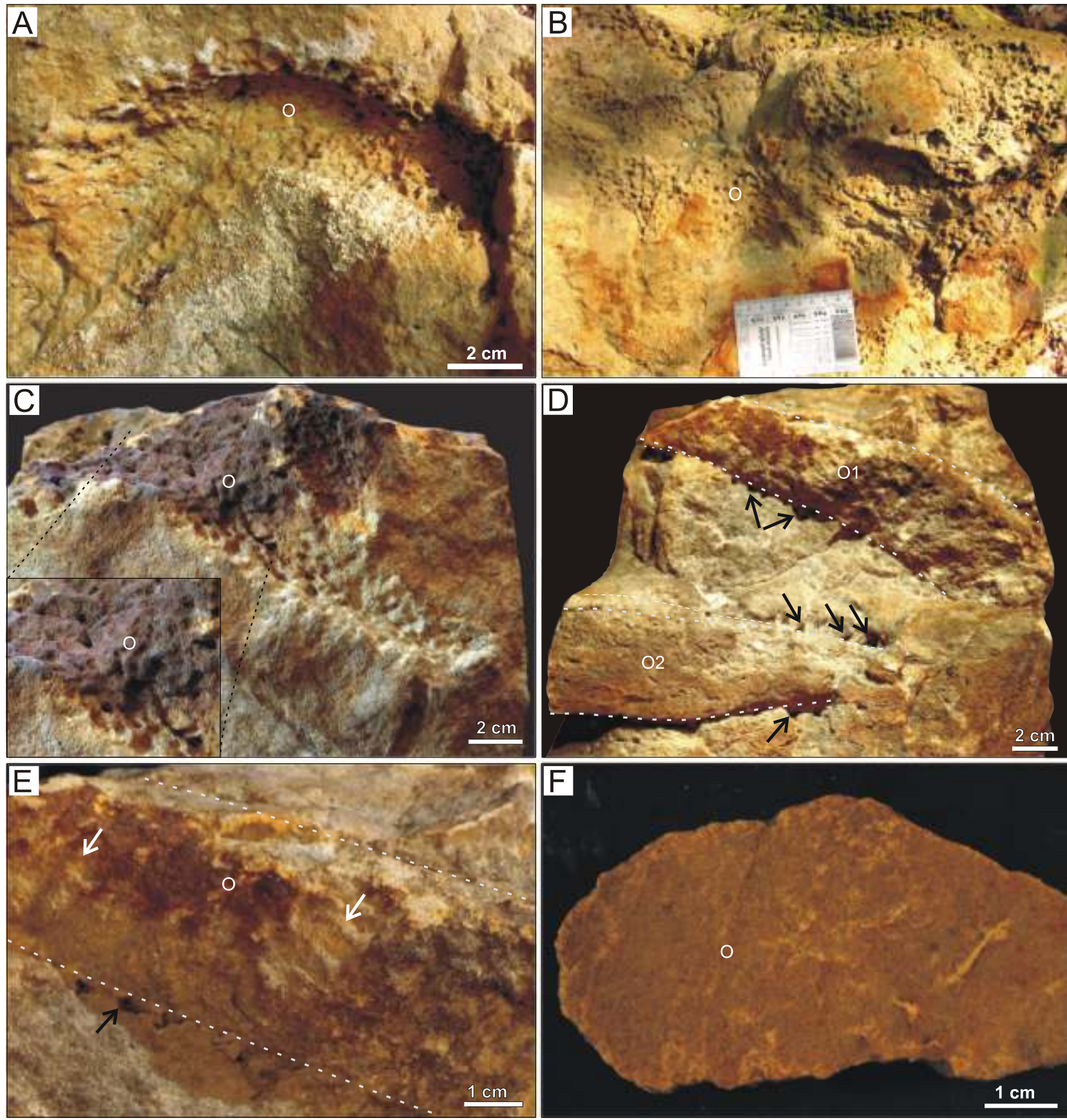

Fig. 22. Ophiomorpha isp. A and its features in the sandstones of erkowice Member at locality 10 (Kotliska); photographs from May 2013

A-C - moulds of burrow surface $(\mathrm{O})$ showing pitting after muddy pellets (remains preserved in some pits) that originally protected the burrow walls from collapse; the complex morphology of the pitted surface in $\mathrm{B}$ is a result of multiple burrowing; $\mathbf{D}$ - casts of a tunnel (O1) and a ?brooding chamber (O2) with dispersed pits after muddy pellets aligned to the burrow surface (arrows); note the nubs in cast O1 and diffuse longitudinal striations in cast $\mathrm{O} 2 ; \mathbf{E}$ - detail of tunnel cast surface $(\mathrm{O})$ showing ridges and nubs (white arrows), as well as pits after clayey pellets at the tunnel margin (black arrow); $\mathbf{F}$ - infill of a ?brood chamber $(\mathrm{O})$ seen in vertical section of the distal part of cast with fragments of host sediment; the faint signs of lamination on the burrow left-hand side and the homogenized sediment at its diffuse right-hand margin suggest a partly active chamber filling with the final infilling by wall collapse; the light coloured streaks enriched in clay may be remnants of destroyed mud pellets

specimen only. It occurs as sand-filled, cylindrical, horizontal to subhorizontal branching tunnels and vertical shafts showing at least partially a lining with agglutinated pelletoidal sediment. Tunnels show high variability in their size, course, pattern and the form of lining (Figs. 17-22). Burrows 10-15 mm thick are most common, with the thickest specimens reaching $40 \mathrm{~mm}$ in diameter. Specimens thicker than $15 \mathrm{~mm}$ are most numerous at locality 11 . Burrow linings, in the form of a chaotic mass of irregular thickness, are always present and vary in the shape and distribution of pellets (Figs. 18A, B, F, G and 20D). Pellets are densely to loosely distributed as sandy globular (Fig. 18A, B) to muddy ovoid, polygonal or conical of various sizes (Fig. 18E, I, 

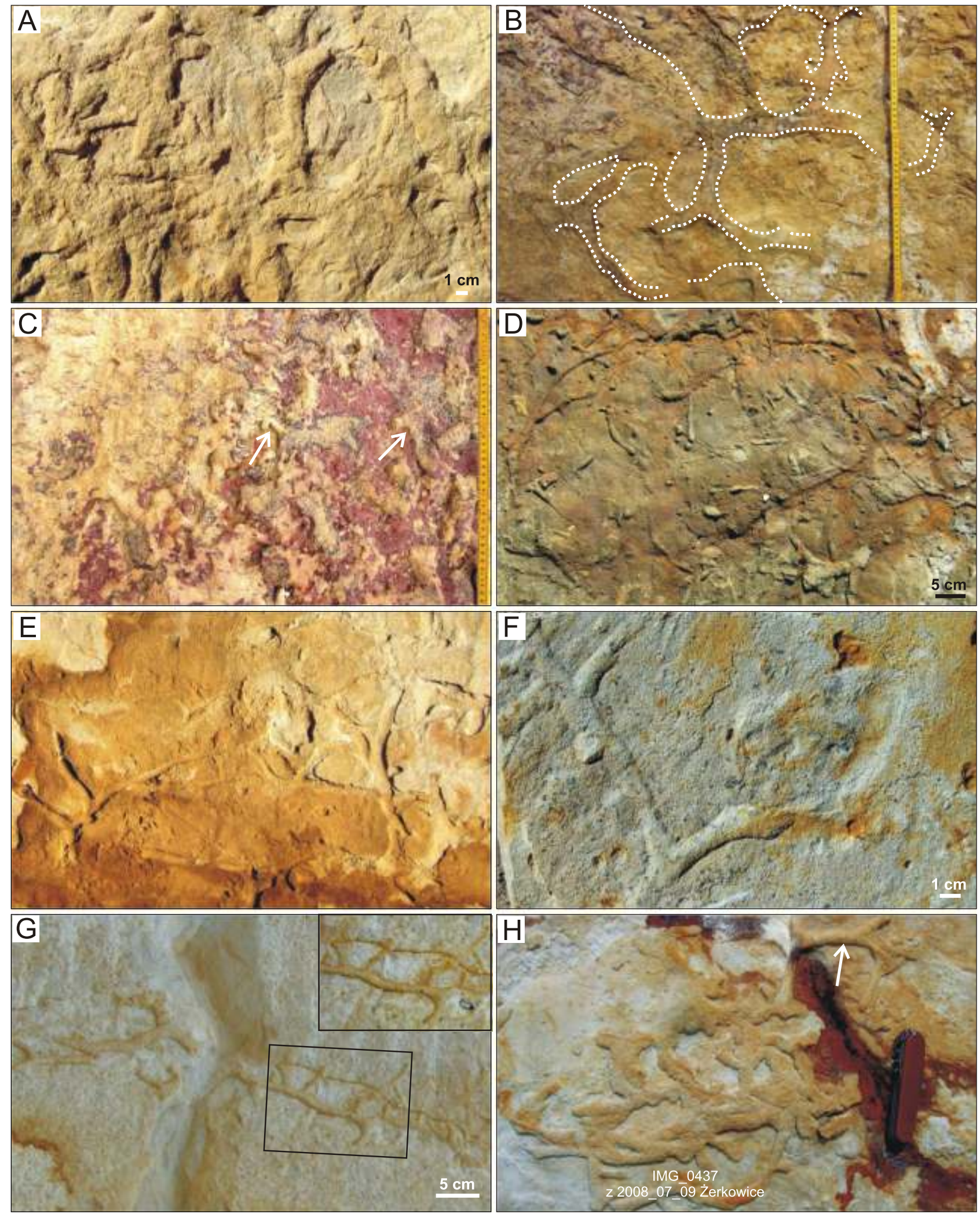

Fig. 23. Occurrence of Thalassinoides in the erkowice Member

A - poorly preserved maze of Thalassinoides paradoxicus in a muddy sandstone at locality 6 (Skała I), photography August 2011; B - poorly preserved maze of Thalassinoides paradoxicus in a heavily bioturbated muddy sandstone at locality 5 (Wartowice), photography August 2011; C - Thalassinoides paradoxicus (arrows) on the top surface of sandstone bed covered with red mudstone at locality 8 ( erkowice), photography August 2008; D - Thalassinoides suevicus on a sandstone bed sole at locality 6 (Skała I), photography August 2011; E Thalassinoides suevicus on the top surface of sandstone bed at locality 6 (Skała I), photography August 2013; F - Thalassinoides suevicus on sandstone bedding surface at locality 9 (Rakowice Małe), photography May 2007; G - Thalassinoides suevicus in the axial part of a scoop-shaped rippled surface separating trough cross-strata sets in a sandstone package at locality 8 ( erkowice), photography July $2008 ; \mathbf{H}$ - Thalassinoides suevicus partly flattened by compaction at the same locality, the pocketknife (scale) is $9 \mathrm{~cm}$ long, photography July 2008 

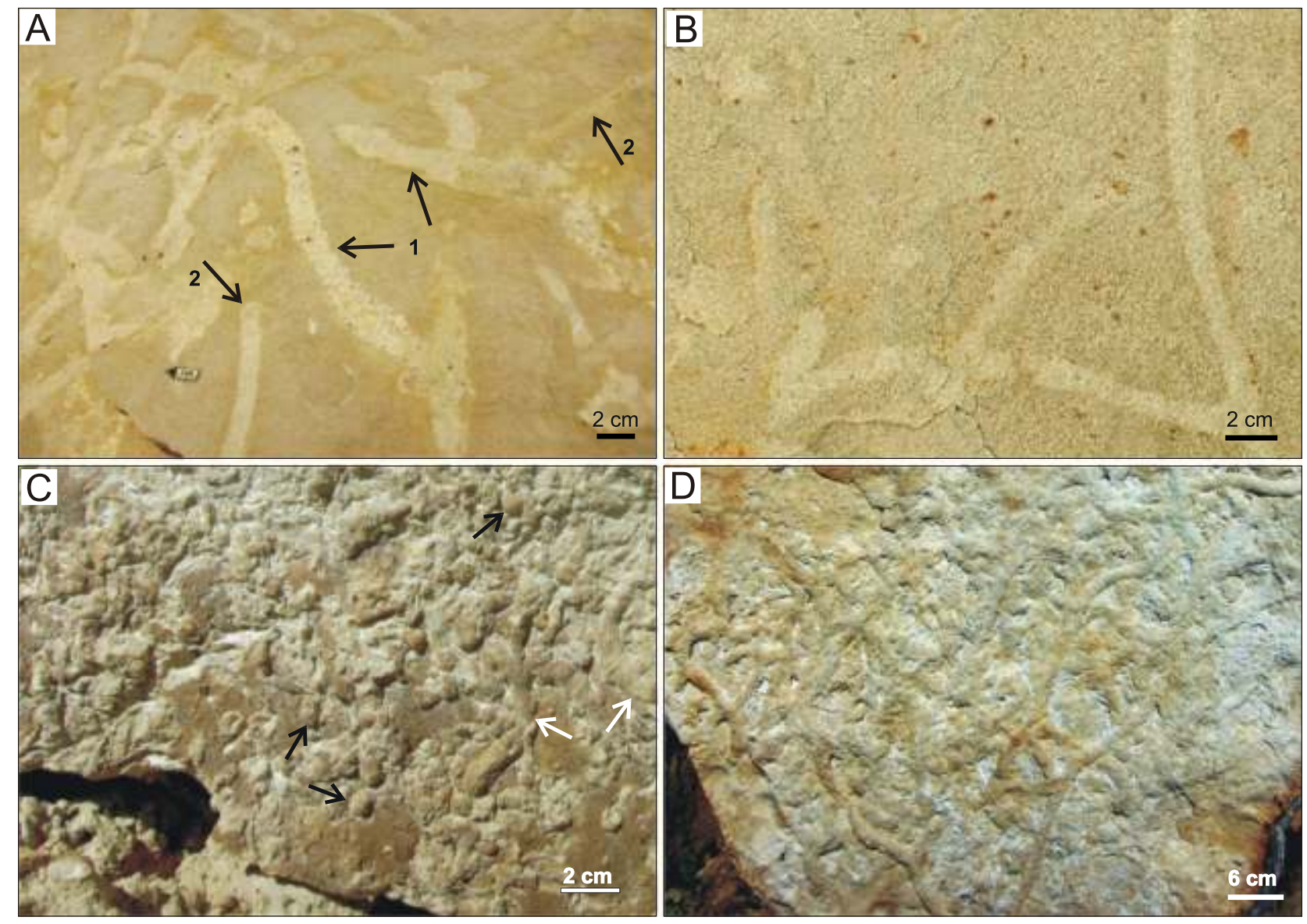

Fig. 24. Controversial trace fossils with Ophiomorpha and Thalassinoides affinity in the erkowice Member

A - Ophiomorpha nodosa (1) and burrows with a straight course and no lining (2), classified tentatively as Thalassinoides isp., locality 5 (Wartowice), photography August 2011; B - Thalassinoides isp. with Y-shaped branching and crisscrossing of burrows, locality 5 (Wartowice), photography October 2010; C -?casts of shallow domichnia or cubichnia (black arrows) and aff. Thalassinoides isp. (white arrows) on the sole of sandstone bed at locality 1B (Czaple), photography July 2015; D - burrows intermediate between Thalassinoides and Ophiomorpha on a sandstone bed sole, showing traces of muddy lining and a rough, knobby tunnel-fill wall, locality 8 ( erkowice), photography May 2008
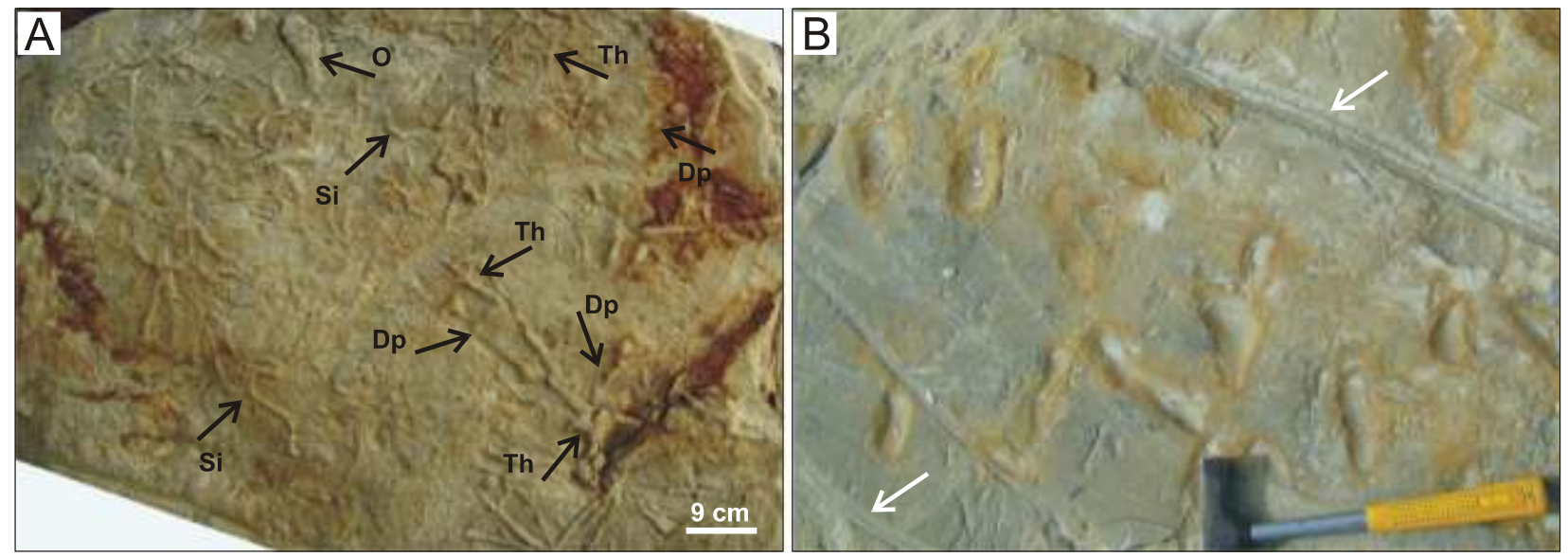

Fig. 25. Selected hypichnia in the erkowice Member

A - ?Diplocraterion isp. (Dp), ?brood chamber of Ophiomorpha isp. (O), ? Sinusichnus isp. (Si) and Thalassinoides isp. (Th) on a sandstone bed sole at locality 8 ( erkowice), photography May 2008; B - Scolicia aff. strozzii (arrows), casts of inverted bivalve shells, small prodmarks and dragmarks on sandstone sole in a loose block at the same locality, photography May 2008 

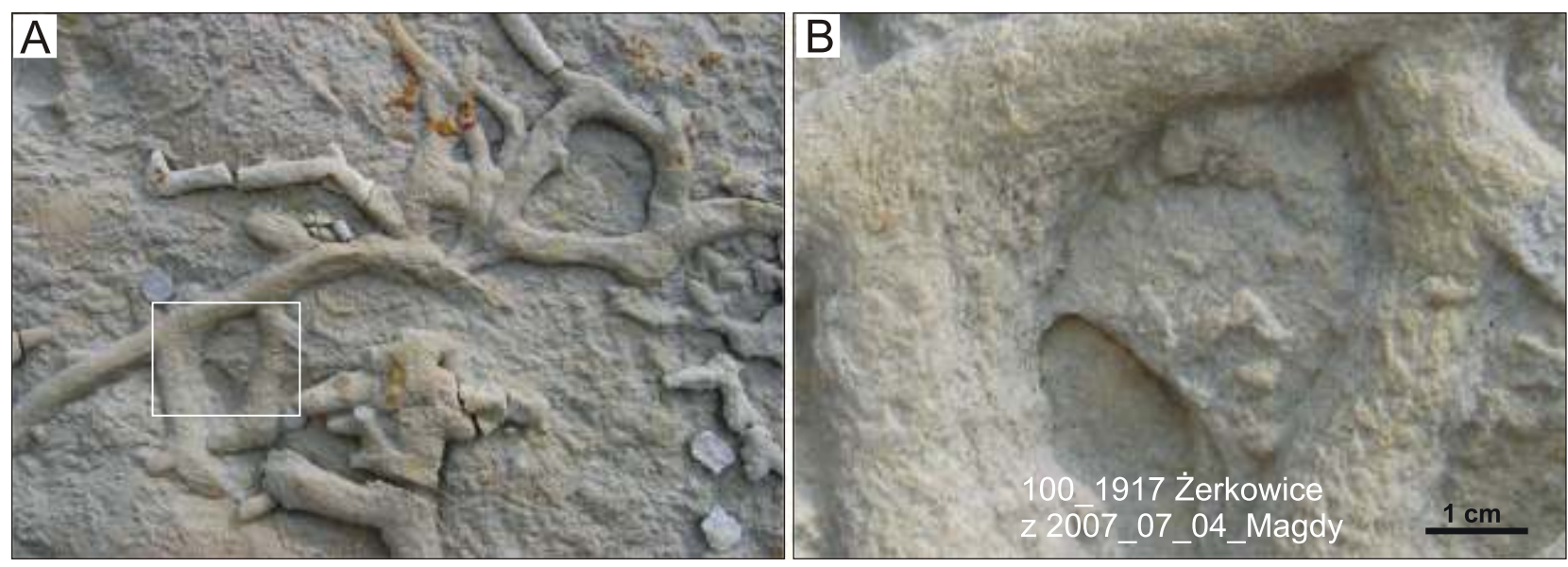

Fig. 26. Spongeliomorpha cf. sudolica in sandstones of the erkowice Member

A - fully exposed burrow system, with the white rectangle indicating detail shown in B and scale as in the latter; B - close-up detail of the burrow in A, showing subtle ornamentation of the burrow walls; locality 8 ( erkowice), photography July 2007

K; cf. Frey et al., 1978; Bromley, 1991; Miller et al., 1998). The lining by a chaotic muddy mass evolves from conical and irregular muddy pellets (Figs. 18G-I and 20D) and is probably the final product of fluidized pellet destruction. The arrangement of pellets changes in some burrows from chaotic to transverse to the burrow axis (Figs. 18C and 19A, B). The wall lining of some burrows is very thin and absent in some of their parts (Fig. 19C, D). Its lack is particularly characteristic of the tunnel floor (Fig. $10 \mathrm{C})$. Notably, the surface of the burrow fill, and thus the interior surface of the wall lining, in the most common Ophiomorpha $10-15 \mathrm{~mm}$ thick appears to be always smooth (Figs. 18A, H, N, 19E, F and 20D). Burrows are typically filled with massive sand. A distinctly meniscate fill was found in only one burrow (Fig. 18L).

Tunnels outnumber shafts by 3 to 1 , except for the outcrop at locality $1 \mathrm{C}$ where rare Ophiomorpha occur mainly as shafts. Vertical distribution of tunnels implies their occurrence typically in an irregular boxwork pattern and subordinately in irregular mazes (cf. Frey and Howard, 1975). Because of unfavourable outcrops, the horizontal pattern of $O$. systems at a scale $>0.5 \mathrm{~m}^{2}$ could be observed only at locality 5 , where most tunnels show a delicately curved, irregular course, a relatively dense branching and a patchy concentration (Fig. 20A-C). Both Y- and T-shaped branching occurs. Some burrow systems show tunnels extending radially from one point (Fig. 20B). The distribution of burrows on surfaces normal and parallel to bedding indicates an Ophiomorpha ichnofabric index (Droser and Bottjer, 1986, 1989) between 1 and 3. A denser distribution is only rarely observed. At localities 5,10 and 11, a high concentration of $O$. occurs in certain parts of the sedimentary succession, giving a typical Ophiomorpha ichnofabric. In other outcrops, $O$. occurs almost exclusively in high dispersion without other trace fossils.

Considering the Ophiomorpha ichnospecies defined in the literature (Uchman, 2009; Knaust, 2017), those observed in the ZMb would appear to represent almost exclusively $O$. nodosa. At locality 5, two small Ophiomorpha systems with distinctly curved to winding burrows, dense branching and densely pelleted wall linings were found (Fig. 21), with pellets of rounded shape and similar size. These burrows are distinctly different from the typical $O$. nodosa and are therefore classified as Ophiomorpha ?nodosa.
Still other Ophiomorpha have been encountered in a thin interval of one sandstone bed at locality 10 . They are recorded mainly as small, irregular, elongate to ramified, conically pitted surfaces up to $\sim 20 \mathrm{~cm}^{2}$ and as sand-filled horizontal to vertical circular burrows, 30-40 mm in diameter, lined with loosely to densely distributed and chaotically oriented, conical muddy pellets (Fig. 22). The burrow fill is massive with a rough surface marked by nubs, short ridges and furrows. The pitted patches are molds of the outer surface of the pelleted burrow lining. Small irregular and partly overlapped patches indicate multiple reworking of burrows. Because of its size and character of lining, this burrow is classified here tentatively as Ophiomorpha isp. A.

One horizontal segment of such a thick $O$. was found associated with a club-shaped sand-built body that appears to bud from it. This body is $14 \mathrm{~cm}$ long and up to $5.5 \mathrm{~cm}$ in diameter, built of fine-grained sand identical to that in the host rock. Its uncovered surface is rough, showing faint longitudinal striations and nubs. Elongate pits perpendicular to the burrow surface occur at the border between its uncovered and hidden part. Some pits show mud remnants inside. The vertical section of the distal burrow part shows its hidden side almost perfectly welded with the host sediment. The infilling sediment shows crude parallel stratification in the sharply outlined, lower part of the burrow and a chaotic sediment above - with an irregular, diffusely outlined and outwards extending, streaky enrichment in mud. Traces of the burrow outline, marked by enrichment in mud, extend at a short distance in continuity with the sharp exposed burrow margin. The club shape suggests that the structure represents a cast of a brooding chamber of Ophiomorpha producers. The burrow was actively filled in the lower part and became closed by a burrow collapse. The streaky enrichment in mud in the chaotic fill seems to comprise remains of destroyed burrow lining. Another specimen of possible Ophiomorpha breeding chamber was found cast on a single surface in sandstone bed at locality 10 (Fig. 22).

The next in frequency, albeit much rarer, in the $\mathrm{ZMb}$ is the ichnogenus Thalassinoides developed as sand-filled, cylindrical, horizontal to subhorizontal, branching, unlined tunnels with some variability of form. Irrespective of their form, the specimens of Thalassinoides as full reliefs or casts were found almost exclusively on sandstone bedding surfaces. Most frequent 

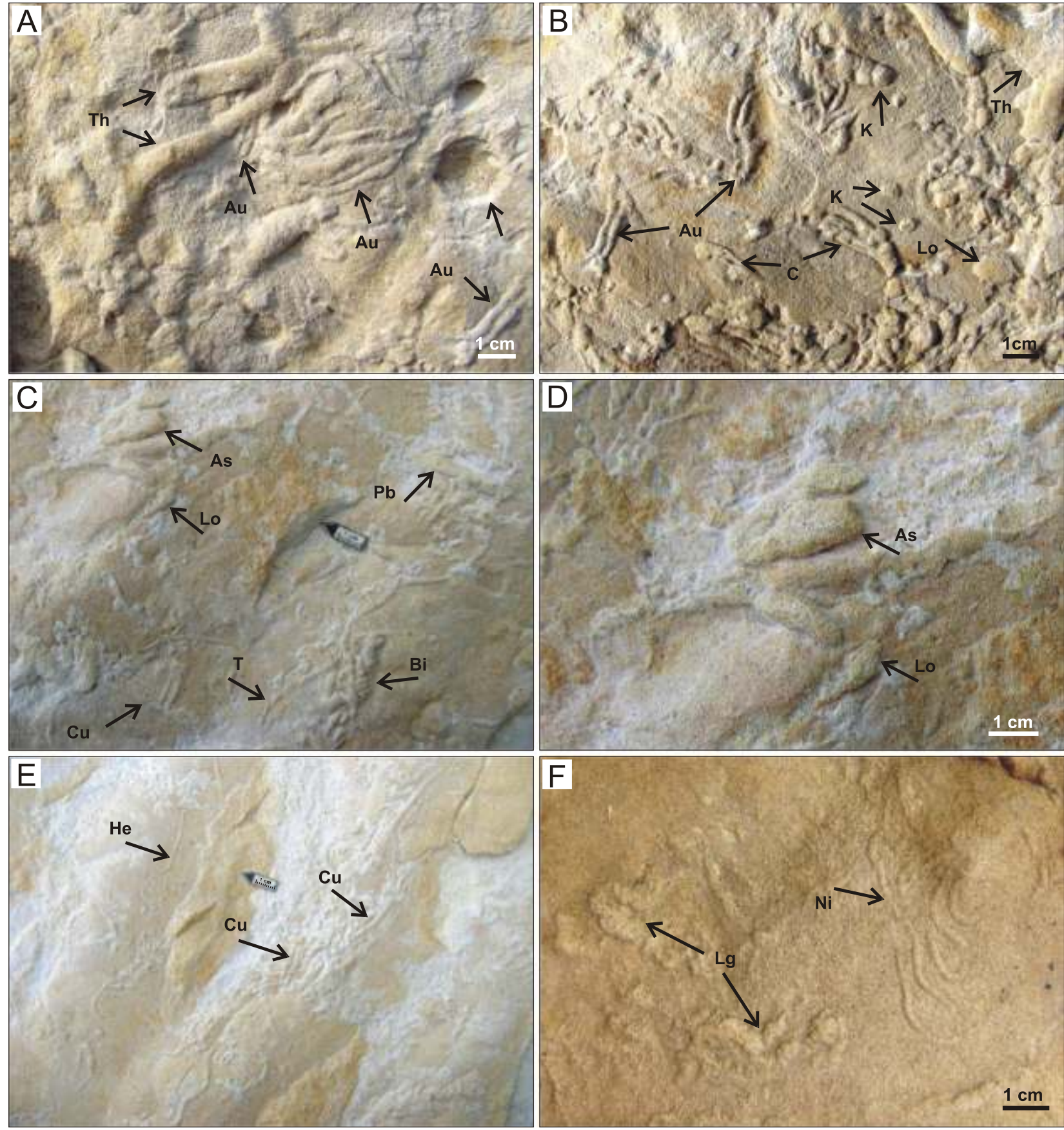

Fig. 27. Other trace fossils on sandstone bedding surfaces in the erowice Member

A - Aulichnites parkerensis ( $\mathrm{Au}$ ) "radiating" from one point and Thalassinoides isp. (Th) on bed top surface, locality 8 ( erkowice), photography May 2008; B - Aulichnites parkerensis (Au), cast of a shallow domichnial hole (K), ?Lockeia isp. (Lo) and Thalassinoides isp. (Th) on sandstone bed top; same locality and time of photograph; C - aff. Asterosoma isp. (As), Biformites insolitus (Bi), ? Curvolithus isp. (Cu), ? Lockeia isp. (Lo) and ? Planolites beverleyensis ( $\mathrm{Pb}$ ) on sandstone bed sole, same locality, photography July 2007; D - close-up detail from C, showing Asterosoma coxii (As) and ? Lockeia isp. (Lo); E - ? Curvolithus isp. (Cu) and Helminthopsis abeli $(\mathrm{He})$ on sandstone bed sole, same locality, photography July 2008; F - ?Nereites irregularis (Ni) and undetermined lumpy leveed groves (Lg) reminiscent of Cerithidea californica trails (Knox and Miller, 1985) on sandstone bed top, locality 5 (Wartowice), photography August 2012

are specimens representing the ichnospecies suevicus and paradoxicus, differing in the style of branching (Fig. 23). Much less common are specimens of a different and somewhat uncertain taxonomic affiliation with Thalassinoides. These include examples encountered in small portions as sand-filled, cylindrical, smooth-walled burrows $\sim 10 \mathrm{~mm}$ in diameter, horizontal to subhorizontal and mainly $Y$-shaped, lacking distinct lining and rarely branched (Figs. 24A, B and 27A, B). They have been classified herein as Thalassinoides isp. Some of them accompany Ophiomorpha nodosa and may represent parts of a complex Ophiomorpha-Thalassinoides burrow system. 

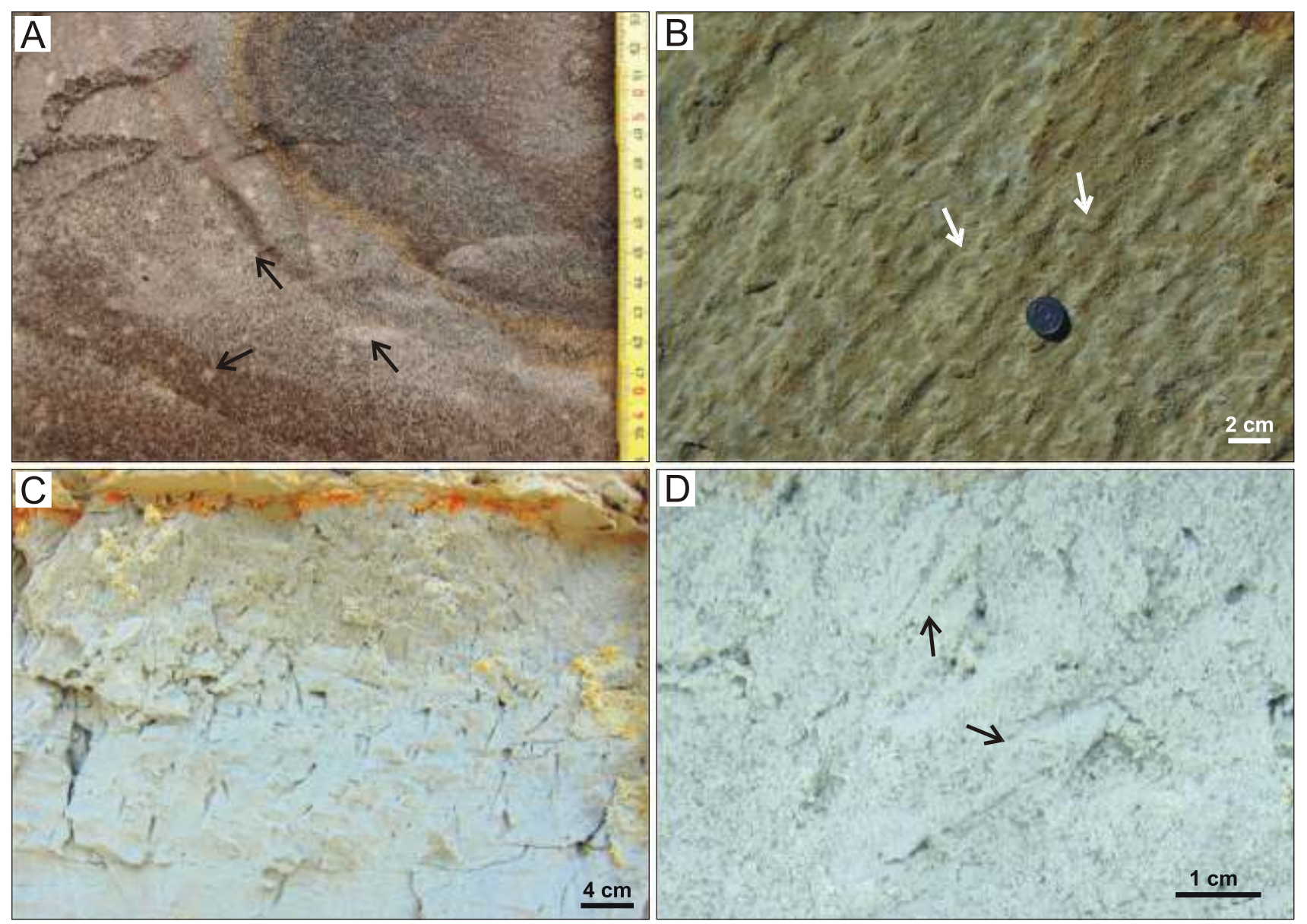

Fig. 28. Accessory endichnial trace fossils in the sandstones of erkowice Member

A - aff. Macaronichnus isp. (arrows) in a sandstone stained with decomposed organic substance, locality 12 (Osiecznica), photography August 2012; B - aff. Sinusichnus isp. (arrows) on sandstone sole, locality 9 (Rakowice Małe), photography May 2007; C, D - ?Rosselia isp. A in vertical section and on bedding plane (arrows), respectively; locality 12 (Osiecznica), photography April 2013

Determined as aff. Thalassinoides isp. were specimens occurring as casts of horizontal, cylindrical, unlined Y-shaped and curved burrows with an irregularly changing diameter of 5-12 $\mathrm{mm}$ and a rough surface (Fig. 24C). They were encountered at locality $1 \mathrm{~B}$ on the sole of a sandstone bed densely covered with casts of undetermined, mostly short, spigot-shaped burrows.

A trace fossil form found as a maze system of cylindrical, Yand T-shaped branched burrows with subtly bumpy surfaces (Fig. 24D) has been classified as intermediate between Ophiomorpha and Thalassinoides.

Bioturbation structures in the CFm are significantly different than in the ZMb (Figs. 14B, E-H, 15A, 16C and 31-39). Only a few undetermined trace-fossil forms, besides mottling structures, were found in the lower, muddy part of the CFm at locality 12 (Fig. 39). It is possible that some of the mottling represents bioturbation. At localities 5 and 9 , in contrast, bioturbation structures occur in nearly whole exposed part of the CFm. At both localities, the basal, mudstone-dominated deposits, up to the upper coal bed, show plant-root structures as the only evident trace fossils (Figs. 14B, E, F, 15A and 16C). The plant-root structures occur as one or more horizons in the basal part of the $\mathrm{CFm}$, directly above the contact with the ZMb at locality 5 and in the seatearth of the lower coal bed at locality 9 . Heavily bioturbated intervals, with $\mathrm{BI}=5$ to 6 and with a particularly rich trace-fossil assemblage at locality 9 (Leszczyński, 2010), occur in the higher part of the CFm succession (Fig. 7).

The outcrop section above the coal bed at locality 5 , up to $\sim 7 \mathrm{~m}$ above the base of CFm, shows Ophiomorpha nodosa mainly as shafts, with some undetermined trace fossils in the heavily bioturbated top parts of sandstone packages overlain by variegated mudstones and in the top parts of some of the individual sandstone beds (Fig. 15B, C, E). Bioturbation pattern (ichnofabric) is reminiscent of Thalassinoides, locally overprinted by structures resembling plant roots and ultimately by dispersed Ophiomorpha isp., O. nodosa occurs solely in sandstones and shows an indistinctly pelleted, white muddy lining (Fig. 15E).

At locality 9, the higher part of the CFm, above the upper coal bed, is the richest in bioturbation structures (Figs. 7, 16C-E, G and 31-38), as documented by Leszczyński (2010). The following ichnogenera were recognized in these deposits: Thalassinoides, Ophiomorpha, Asterosoma, Palaeophycus, Planolites, Skolithos, Teredolites, Chondrites, Cylindrichnus, Arenicolites, Rosselia, Taenidium, Teichichnus, Phycodes, Phycosiphon and Schaubcylindrichnus. The author's subsequent study has led to a slight modification of this documentation. The number of ichnogenera is the same (Table 2), but Phycodes and Phycosiphon have been excluded, while Helminthopsis and aff. Macaronichnus have been added as new 

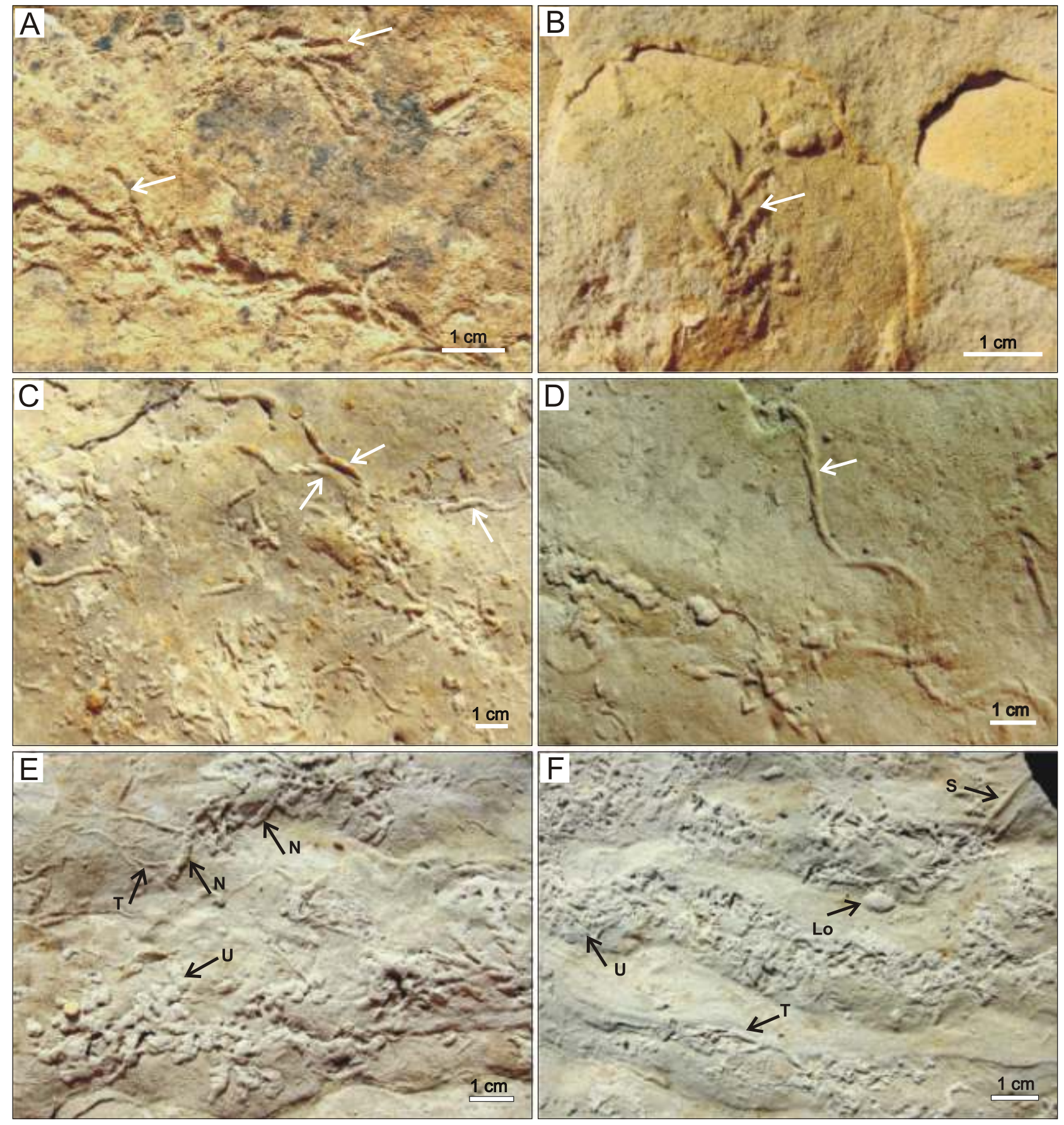

Fig. 29. Trace fossils on sandstone bedding surfaces in the erkowice Member

A - Ophioichnus isp. (arrows) on sandstone bed top (arrow), locality 5 (Wartowice), photography August 2017; B - Taxichnites ? wurmi (arrow) on sandstone bed sole, same locality, photography July 2013; C - Treptichnus aff. bifurcatus (arrows) and undetermined burrows on sandstone bed sole, locality 6 (Skała I), photography July 2013; D - Treptichnus aff. bifurcatus (arrow) on sandstone bed sole, locality 6 (Skała I), photography July 2013; E - ?Nereites isp. (Dreginozoum preservation, N), cf. Treptichnus isp. (T) and undetermined burrows (U) on sandstone bed sole, same locality, photography July 2013; F - ? Lockeia isp. (Lo), aff. Scolicia isp. (S), Treptichnus isp. $(\mathrm{T})$ and undetermined burrows $(\mathrm{U})$ on sandstone bed sole, erkowice Member, same locality and photography date

ichnogenera. The occurrence of Rosselia, Schaubcylindrichnus, Taenidium and Teichichnus remains uncertain. Some of the ichnotaxa classified formally in Leszczyński (2010) have now been classified in open nomenclature (e.g., Palaeophycus and Planolites). The structures of clustered burrows classified herein as aff. Thalassinoides isp. were interpreted earlier
(Leszczyński, 2010) as ichnogenus Phycodes. Likewise, burrows with a curved course and thick lamination in vertical cross-section (Fig. 16D), classified herein as ?Teichichnus isp., were earlier interpreted as Teichichnus zigzag. Their curved vertical course is now considered as due to deformation by a high compaction of the host peaty sediment (presently coal). 

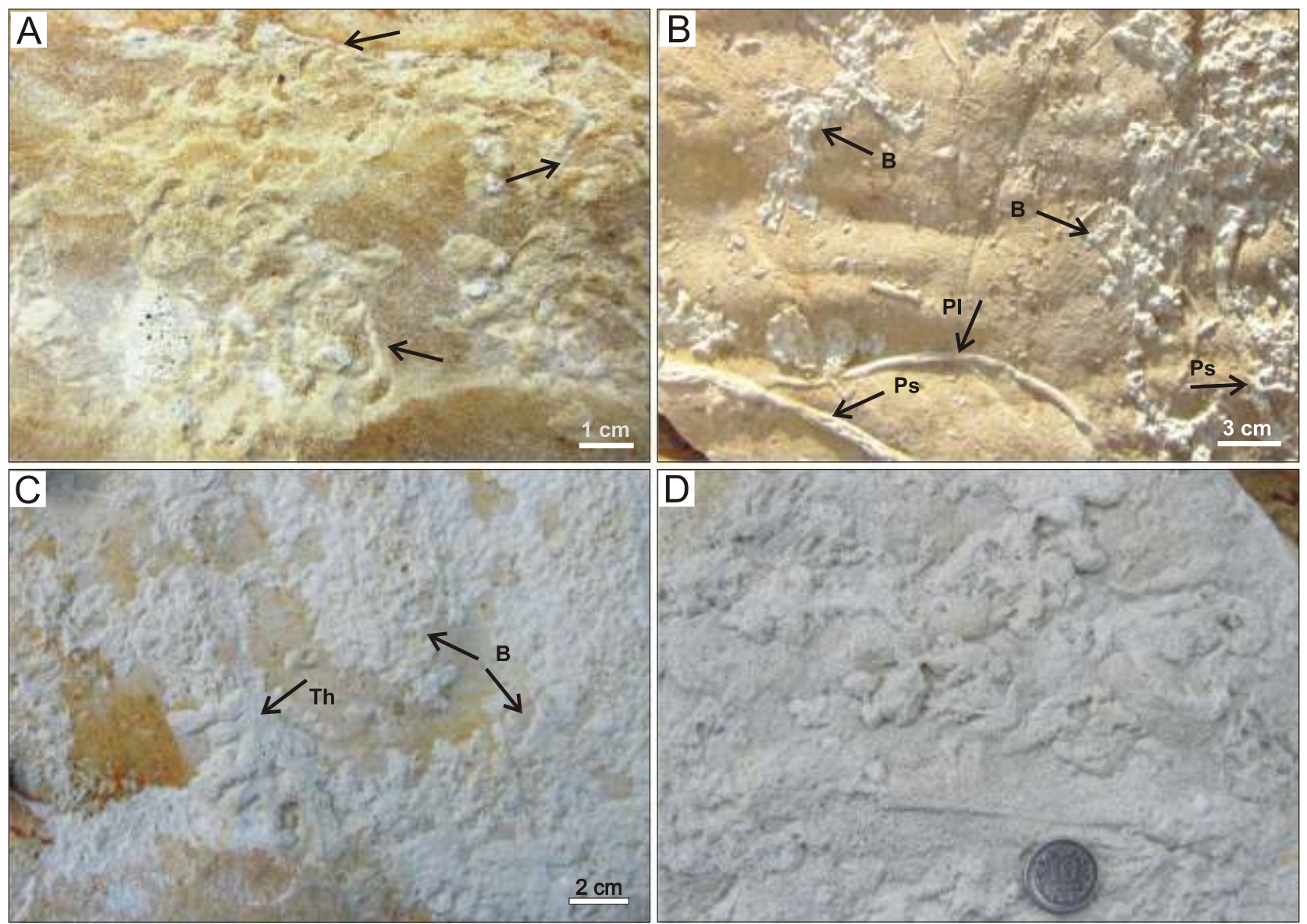

Fig. 30. Undetermined trace fossils and suspected biogenic features on sandstone bedding surfaces in the erkowice Member

A - bunches and panicles of cane-patterned reliefs (arrows) on a rippled top surface of sandstone bed, locality 5 (Wartowice), photography August 2011; B - Palaeophycus sulcatus (Ps), Planolites beverleyensis (PI) and undetermined pockmarked relief produced by scavengers (B) on sandstone bed sole with wave-ripple casts, same locality and photography date; C - minute ? Thalassinoides isp. (Th) deformed by compaction and patches of undetermined curly lumps produced by unknown scavenger $(B)$ on sandstone bed sole, locality 8 ( erkowice), photography July 2008; D - irregular projection of undetermined reliefs on sandstone bed sole, locality 9 (Rakowice Małe), photography July 2007

The influence of sediment compaction is also indicated by a highly compressed shape of the casts of vertically oriented Teredolites clavatus borings.

The trace fossils classified as aff. Thalassionoides isp. are specific in showing an irregular outline, a rough wall and forms of two sizes. Thin forms (7-8 $\mathrm{mm}$ in diameter) occur in fragments up to $2 \mathrm{~cm}$ long, whereas the thicker ones attain $7 \mathrm{~cm}$ in length. Moreover, the thin forms appear to sprout from the thick ones. Based on their size and branching relationship, both forms are considered as an unidentified species close to the ichnogenus Thallasinoides, for which the offshooting of small forms from larger ones is known (e.g., Howard and Frey, 1984; Knaust, 2017). The small forms are considered to have been produced by juvenile animals of the same endobenthic species (Frey and Howard, 1975).

In total, 27 ichnotaxa have been identified in this part of the CFm at locality 9, with one trace-fossil form distinguished as undetermined. The abrupt change in bioturbation style recorded in the upper coal bed at locality 9 is marked by the occurrence of numerous sand-built endichnia, including Thalassinoides suevicus, aff. Thalassionoides isp., ?Teichichnus isp. (Fig. 16D) and undetermined obliquely-lamelled structures (Fig. 32D), as well as abundant epichnial Teredolites clavatus (Figs. 7 and
12F). This trace-fossil assemblage constrasts with the delicate plant-root traces of shrub-like vegetation that are the only macroscopically recognizable biotic structures in the seatearth beneath the coal bed (Fig. 16C).

A heavy bioturbation ( $\mathrm{BI}=5$ to 6 ) is shown by argillaceous sandstone and heterolithic deposits overlying the upper coal bed at locality 9. The dominant ichnofabric combines ?Teichichnus and ?Asterosoma (Fig. 34), with dispersed Cylindrichnus concentricus (Fig. 36A-D), ?Palaeophycus heberti (Figs. 35C, D and 36C), ?Palaeophycus isp. (Fig. 35A, C-E), ?Planolites isp. (Fig. 35A, E, F), single ?Schaubcylindrichnus isp. (Fig. 37D), ?Taenidium baretti (Fig. 38A, B), ?Taenidium isp. A (Fig. 38C), ?Taenidium isp. B (Fig. 38D) and ?Rosselia isp. (Fig. 36F). The bioturbation intensity, variability and burrow size decrease upwards, which is associated with an upward decrease of sandstone content and disappearance of ichnofabric dominated by ?Teichichnus and ?Asterosoma. The higher part of the heterolithic package shows levels of densely distributed Helminthopsis abeli (Fig. 37C, D), aff. Macaronichnus isp. (Fig. 36E) and Arenicolites sparsus (Fig. 37A).

These heterolithic deposits pass upwards into a package of clayey mudstones showing swirl-type structures, which may at least partly be of biogenic origin. The next abrupt change in 

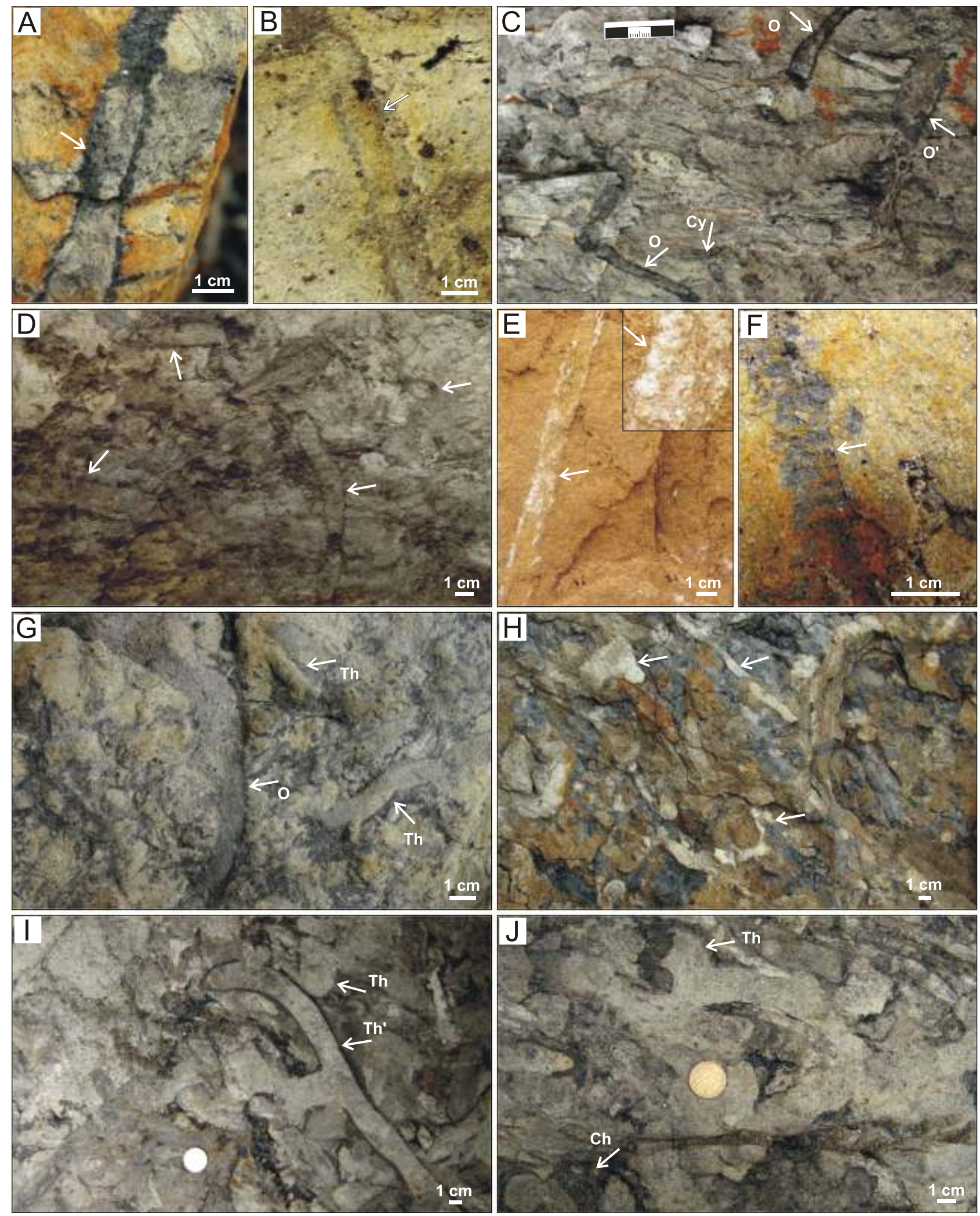

Fig. 31. Ophiomorpha, Thalassinoides and associated trace fossils in the Czerna Formation

A, B - Ophiomorpha nodosa tunnel (arrow) with an irregularly mud pellet-lined wall, locality 9 (Rakowice Małe), photography August 2009 (A) September 2008 (B); C - highly bioturbated heterolithic deposit with Cylindrichnus isp. (Cy), Ophiomorpha nodosa (O) and Ophiomorpha isp. (O'), same locality, photography August 2009; D - poorly preserved Ophiomorpha? nodosa (arrows) in heavily bioturbated muddy sandstone, same locality and photography date; E - Ophiomorpha nodosa shaft (arrow) with irregular white muddy pelleted lining, locality 5 (Wartowice), photography August 2011; F - Ophiomorpha annulata shaft (arrow) lined with elongate pellets rich in organic matter, locality 9 (Rakowice Małe), photography September 2008; G - Thalassinoides suevicus (Th) and Ophiomorpha nodosa (O) on lower surface of thin sandstone bed, same locality, photography August 2009; H - Thalassinoides ? suevicus (arrow) in heavily bioturbated heterolithic deposit, same locality and photography date; I- Thalassinoides suevicus (Th) and ? Thalassinoides suevicus showing local mud lining (Th') in highly bioturbated sand-dominated heterolithic deposit, same locality, photography May 2008; J - Thalassinoides suevicus (Th) and Chondrites intricatus (Ch) in a carbonaceous mudstone, same locality, photography May 2008 

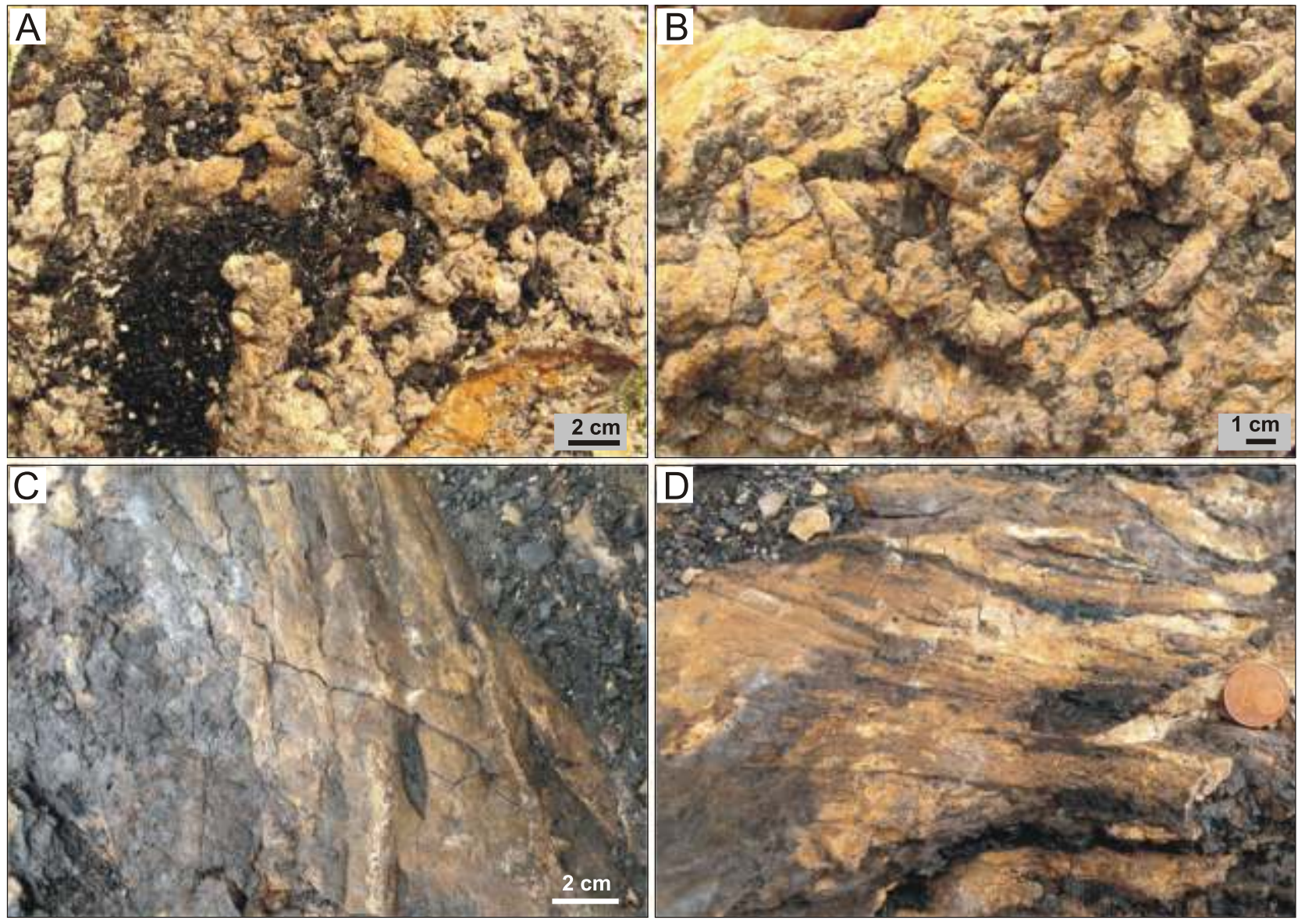

Fig. 32. Trace fossils in coal in the Nowogrodziec Member of the Czerna Formation

A - aff. Thalassinoides isp., locality 9 (Rakowice Małe), photography August 2008; B - aff. Thalassinoides isp., same locality and photography date; C - highly compacted maze of Thalassinoides suevicus, same locality, photography August 2009; D - taxonomically undetermined obliquely lamellated structure (arrow) unclearly associated with Thalassinoides-like burrows, same locality and photography date

bioturbation style is in the overlying layer of coaly mudstone, which is dominated by distinct, sand-built trace fossils including Chondrites intricatus in the basal part (Figs. 33F and 37B) and - towards the top - cross-cut by Asterosoma ludvigae (Fig. 33), Thalassinoides isp. (Fig. 33) and Palaeophycus tubularis (Fig. $33 \mathrm{E})$, listed in their sequence of mutual cross-cutting. A specific feature of the trace-fossil assemblage in the coaly mudstone is occurrence of burrows that show a Thalassinoides pattern, but are lined irregularly with mudstone similar to the host sediment (Fig. 31I), which suggests that the lining may have been formed passively by the soiling of tunnel walls through a multiple transit of the animal. Therefore, such trace fossils have been classified as ? Thalassinoides suevicus.

The overlying part of the CFm exposed at locality 9, composed of heterolithic deposits fluctuating between sandstoneand mudstone-dominated and enclosing wedges of low-angle stratified sandstone up to $30 \mathrm{~cm}$ thick, shows poorly preserved trace fossils including Asterosoma, ?Taenidium, ?Teichichnus, Thalassinoides suevicus, Ophiomorpha nodosa, O. annulata, ?Planolites isp. and Palaeophycus isp. The sandy topmost part of the outcrop section shows Skolithos linearis and rare ?Arenicolites isp., as well as numerous bivalve casts and imprints.

A subordinate but characteristic feature of the CFm at locality 9 is the occurrence of Ophiomorpha with exclusively dark grey to black linings composed of dispersed, irregular, spiky conical pellets (cf. Pollard et al., 1993; Pedersen and Bromley, 2006) and an uneven sediment mass. In contrast to its form at locality 5, the Ophiomorpha at locality 9 occurs mainly as tunnels (galleries). Burrow fill is lithologically variable, massive, sporadically meniscate. Single specimens of $O$. annulata also occur. In the earlier study (Leszczyński, 2010), Ophiomorpha with irregular, pelleted mud linings was interpreted as $O$. irregulaire, but is herein reconsidered as $O$. nodosa.

\section{DISCUSSION}

\section{LITHOFACIES RECORD OF ERKOWICE MB. SEDIMENTATION}

Physical sedimentary structures of the ZMb sandstones indicate a whole range of hydraulic conditions. The trough cross-stratification (3D dunes), planar cross-stratification (2D dunes), planar parallel stratification (plane-bed transport) and ripple cross-lamination (ripple marks) indicate unidirectional currents of lower to upper flow regime (Harms et al., 1975, 1982; Allen, 1982). Dune foresets indicate variable palaeocurrent directions, 

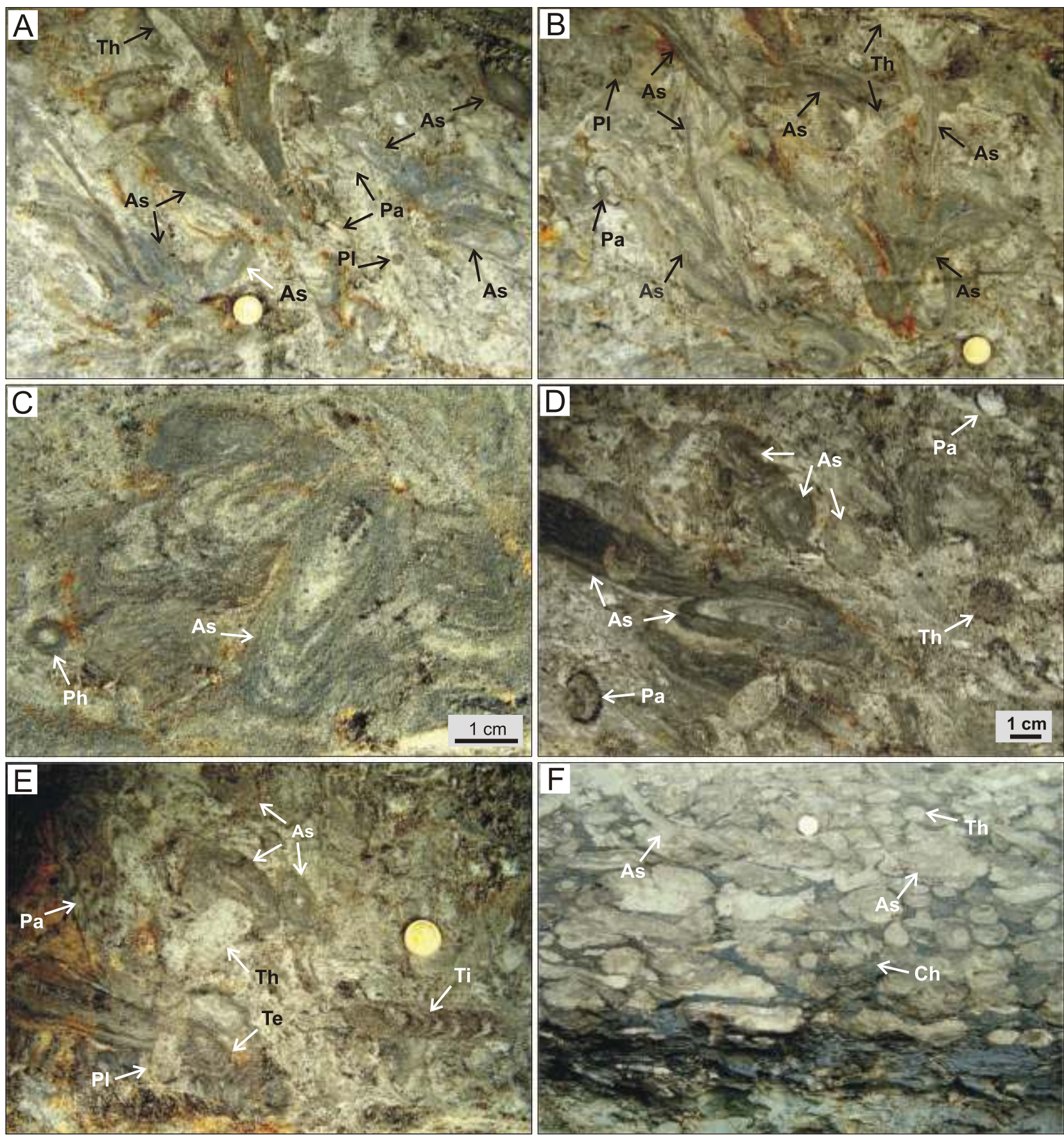

Fig. 33. Asterosoma ludvigae and associated trace fossils in the Nowogrodziec Member of the Czerna Formation

Plane views $(\mathbf{A}-\mathbf{E})$ and vertical section $(\mathbf{F})$ of a combined Asterosoma-Thalassinoides-Chondrites ichnofabric in carbonaceous mudstones, including ? Asterosoma isp. (As), Chondrites intricatus (Ch), Palaeophycus ?tubularis (Pa), ?Palaeophycus heberti (Ph), Planolites isp. (PI), ? Taenidium crassum (Ti) and Thalassinoides isp. (Th); coin diameter (scale) is $1.5 \mathrm{~cm}$, locality 9 (Rakowice Małe), photography May 2008

partly reversing, oriented mainly towards the NW and SE, which means parallel to the basin axis. The evidence of current reversals supports the notion of tidal currents (Bassyouni, 1984), which in the basin's littoral environment combined probably with sediment drift by alongshore currents. Piles of vertically stacked dune cross-sets, several metres thick and with reactivation surfaces, are thought to represent longitudinal tidal bars (cf. Allen, 1982; Longhitano and Nemec, 2005; Messina et al., 2014). Large isolated sets of planar cross-strata, a few metres thick, are probably transverse tidal bars (cf. Allen, 1982). Occasional claystone, mudstone or siltstone drapes between cross-strata sets, as well as the thin interbeds of mud-draped ripple cross-lamination at the scoop-shaped bases of trough cross-sets, indicate suspension fallout in the hydraulic shadow of high-relief bedforms.

Wedges of basinwards-inclined low-angle planar parallel stratification represent episodic encroachment of foreshore swash zone and some wave-worked mouth bars of a shoal-wa- 

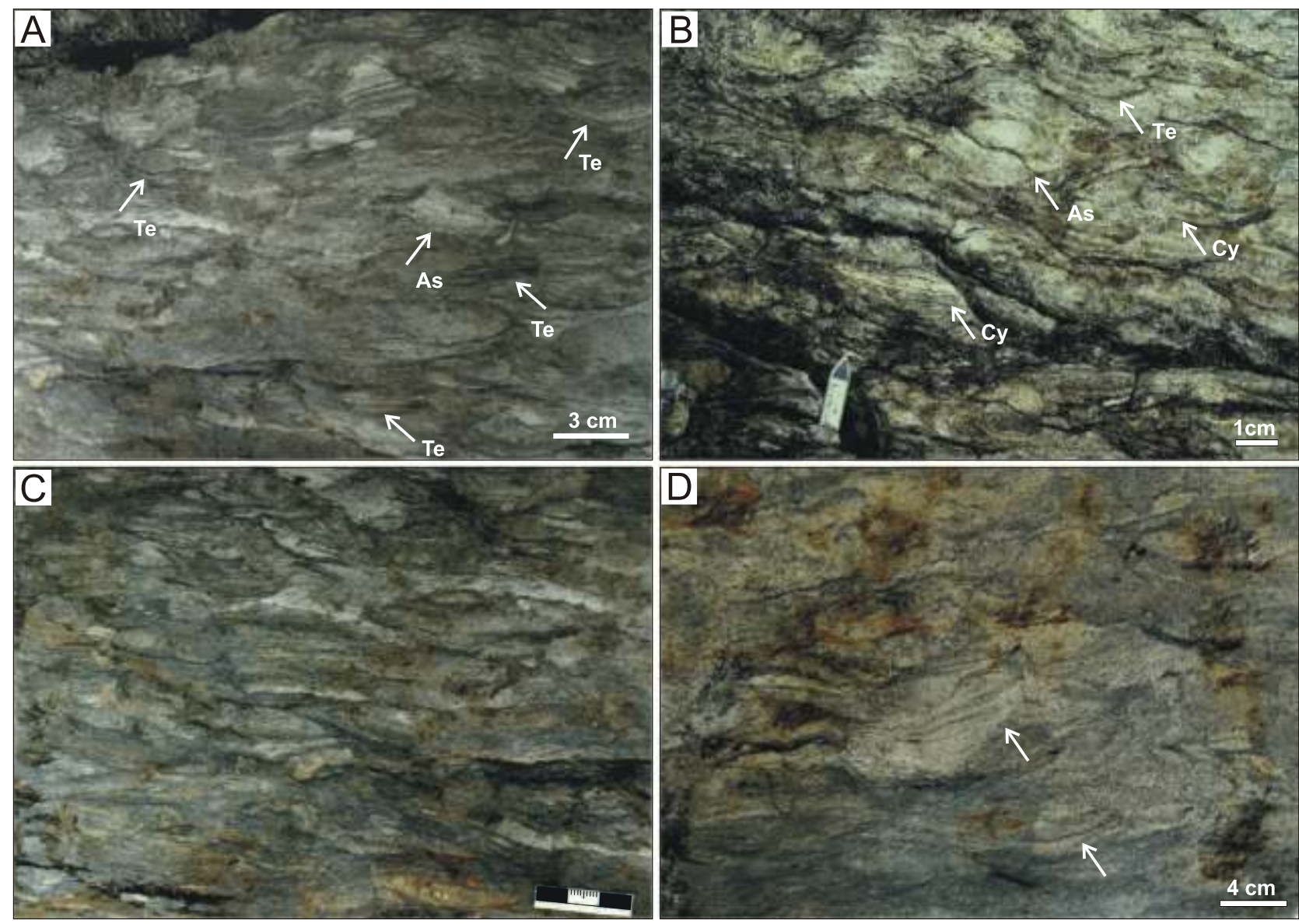

Fig. 34. Ichnofabric in completely bioturbated heterolithic deposits of the Nowogrodziec Member of the Czerna Formation, recording mass occurrence of spreited and laminated trace fossils (Teichichnus isp., Asterosoma isp. and Cylindrichnus isp.)

Vertical outcrop sections showing: A - ichnofabric dominated by ?Teichichnus isp. (Te) and ?Asterosoma isp. (As), locality 9 (Rakowice Małe), photography August 2009; B - ?Asterosoma isp. (As), Cylindrichnus isp. (Cy) and ? Teichichnus isp. (Te), same locality, photography July 2007; C - ichnofabric dominated by ?Teichichnus isp., scale black and white bars are $1 \mathrm{~cm}$, same locality and photography date; D - ? Teichichnus isp. (arrows), same locality, photography August 2009

ter delta (cf. Ilgar and Nemec, 2005; Leszczyński and Nemec, 2015). Plane-parallel stratification intercalated with symmetrical and asymmetrical ripple cross-lamination (2D wave ripples) represents the upper shoreface zone (cf. Komar and Miller, 1975; Harms et al., 1982), with the associated solitary sets of planar cross-stratification interpreted as longshore bars (cf. Allen, 1982). Massive sandstones, if not homogenized by bioturbation, are probably a result of sediment liquefaction - perhaps in situ, without displacement, where lacking a sharp base. Sharp-based, thinner and normal-graded sandstone beds may represent liquefied gravity flows derived from large bedform collapses or be a product of the fallout of transient sand suspension clouds generated by storm events. Evidence of hydroplastically folded cross-strata supports the notion of large bedform morphological instability and possible collapses.

Taken together, the lithofacies of the ZMb indicate littoral sedimentation in a basin-wide shoreface environment, with tidal bars formed at the basin axis and with an episodic encroachment of the foreshore zone and shoal-water deltaic mouth bars. Deltaic deposits occur in the highest part of the ZMb at localities $1 \mathrm{~B}, 5$ and 12, apparently marking the final stage of the mid-Coniacian regressive phase in the basin. The shingled lenticular mouth-bar bodies, more than hundred metres wide, are locally topped with deposits of distributary channels and vegetated interchannel areas. Because of their significant admixture of coarse sand, these deltaic sandstones at locality 5 are included in the mine terminology to the overlying CFm, but are herein considered an integral part of the regressive ZMb succession.

The lithosome of mouth-bar sandstones at localities $1 \mathrm{~B}$ and 5 (see Fig. 7A, at $\sim 11 \mathrm{~m}$ ) is separated from the underlying sandstones by a package of mudstones or mudstone-dominated heterolithic deposits, up to $60 \mathrm{~cm}$ thick. The top of the underlying sandstones is completely bioturbated, with a high concentration of Ophiomorpha decreasing upwards and overprinted by plant roots. The evidence suggests an episode of sedimentation in a transient backshore lagoon, probably sheltered by beach-ridge growth and ultimately emerged. This unique local record of short-term shoreline shifts indicates a stepwise nature of the bulk regression, as the emerged lagoon area must have been drowned for the delta progradation to occur.

Thick cosets of dune-scale cross-stratification in outcrops $1 \mathrm{C}$ and 1D, interpreted as large tidal bars, are in the area directly outside a hypothetical strait - between the East Sudetic and West Sudetic islands - which at the time of ZMb sedimentation was still connecting the North Sudetic Basin with the 

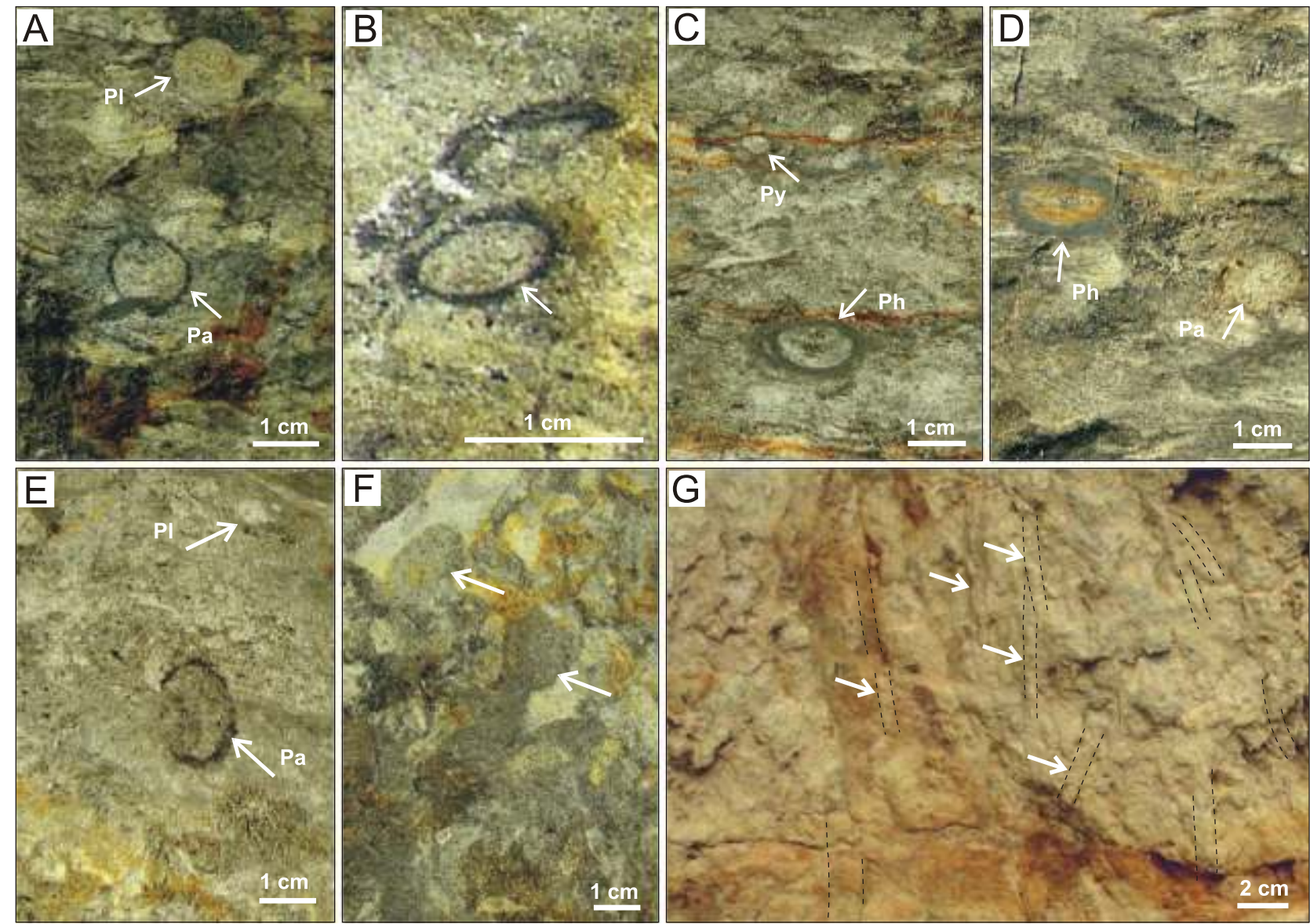

Fig. 35. Trace fossils in vertical sections of bioturbated heterolithic deposits above the basal coal bed in the Nowogrodziec Member of the Czerna Formation at locality 9 (Rakowice Małe)

A - ? Palaeophycus isp. (Pa) and ?Planolites isp. (PI), photography August 2009; B - ?Palaeophycus isp. (arrow), photography May 2008; C - ? Palaeophycus heberti (Ph) and ? Phycodes palmatum (Py), same photography date; D - ? Palaeophycus heberti (Ph) and ?Palaeophycus isp. (Pa), same photography date; $\mathrm{E}$ - ? Palaeophycus isp. ( $\mathrm{Pa}$ ) and ?Planolites isp. (PI), same photography date May; F-? Planolites isp. (arrows), photography September 2009; G - Skolithos linearis (accentuated by dashed outlines and some also indicated by arrows) fragmentarily preserved in a heavily bioturbated sandstone, photography August 2009

realm of the Intrasudetic and Bohemian basins to the south (cf. Milewicz, 1997; Voigt et al., 2008). The strait confinement would greatly enhance tidal currents, which might explain the development of prominent tidal bars. One direction of dune transport would likely dominate in such a setting, while the perennial action of waves would keep the mud suspension afloat and prevent formation of mud drapes on dune foresets (cf. Longhitano and Nemec, 2005). No relics of the strait-fill deposits are preserved today, but one might speculate that as the strait became emerged by the mid-Coniacian regression, it probably turned into a valley with a fluvial system that formed a shoal-water delta in the study area.

A marked impact of tides has been well-documented in the Turonian deposits of the Bohemian Basin by Mitchell et al. (2010), with a cursory consideration of the North Sudetic Basin. Estimates from the Imperial College Ocean Model (Mitchell et al., 2010) have predicted occurrence of a microtidal to mesotidal regime in the Bohemian Basin, with increased bed-shear stress of tidal currents in local embayments and straits. Similar conditions might be expected in the Coniacian North Sudetic Basin, where basin elongation would also considerable enhance tidal currents.

\section{TRACE-FOSSIL RECORD OF ERKOWICE MB. SEDIMENTATION}

The trace-fossil assemblages and distribution of bioturbation structures in the ZMb provide significant additional information on the Coniacian palaeoenvironment and sedimentation conditions in the North Sudetic Basin. The erratic occurrence of bioturbation structures and dominance of non-bioturbated sandstones indicates that the sand deposition was generally fast, and too vigorous for the seafloor to be extensively colonized by benthos (cf. Reineck and Singh, 1980; Mŕngano and Buatois, 2004), and that the bioturbation occurred only occasionally and in limited areas. The occurrence of bioturbation structures mainly at sandstone bed sharp boundaries, or in the top parts of a bed overlain by mudstone, claystone or heterolithic deposits, indicates a specific type of narrow, short-lived colonization windows: the top surfaces of frontally migrating bedforms, such as dunes and bars; the sheltered forefront areas of the bedforms hydraulic shadow, where also sparse local deposition of mud occurred; and the low-energy bottom areas created by episodes of relative sea-level rise or sheltered as a local transient lagoon (cf. Pollard et al., 1993; Bromley, 1996; 

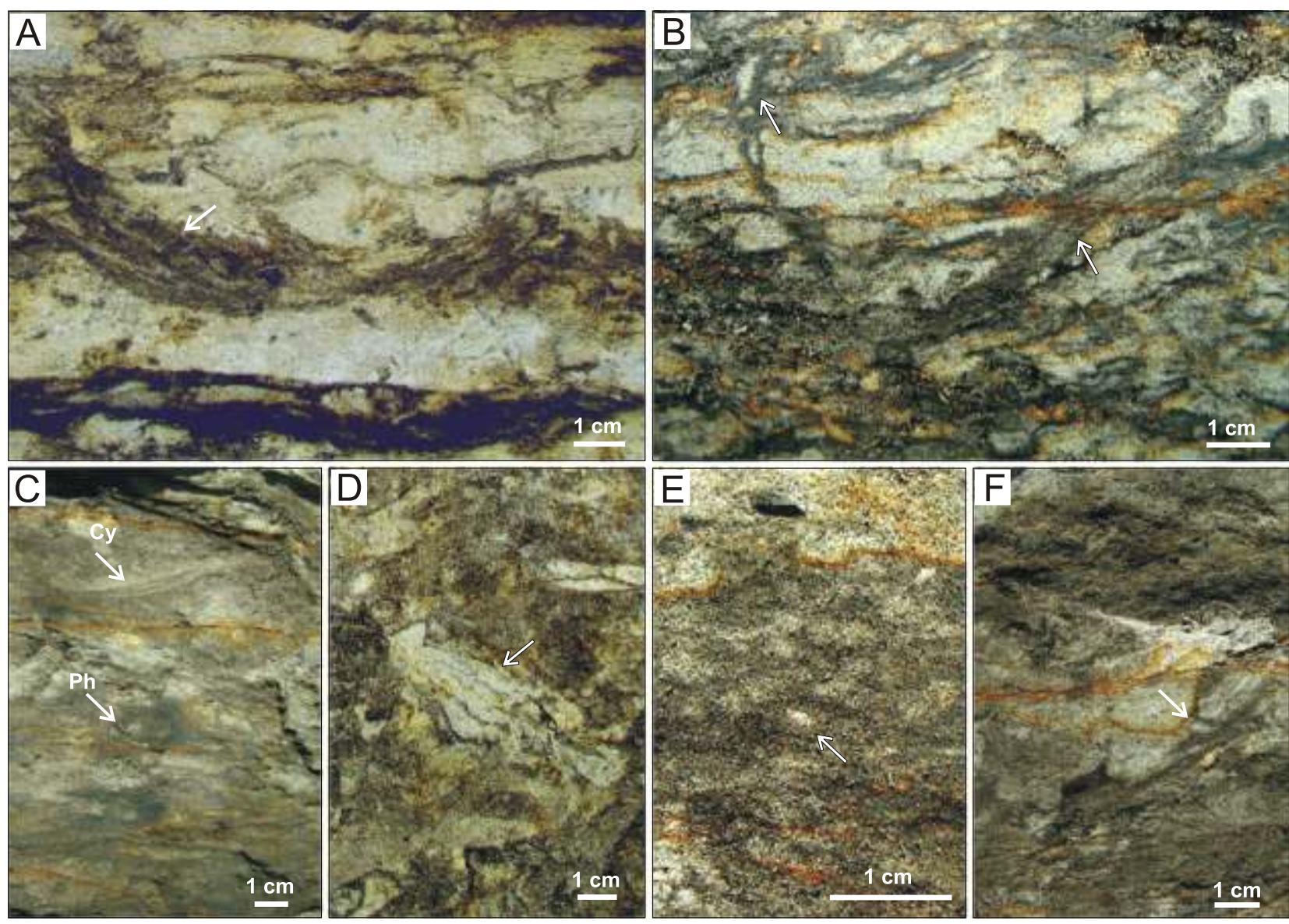

Fig. 36. Other trace fossils in vertical sections of bioturbated heterolithic deposits above the basal coal bed in the Nowogrodziec Member of the Czerna Formation at locality 9 (Rakowice Małe)

A - Cylindrichnus concentricus (arrow) in sandstone-dominated heterolithic deposit, photography September 2008; B - bedding oblique and nearly horizontal Cylindrichnus concentricus burrows (arrows) in sandstone-dominated heterolithic deposit, photography May 2008; C - nearly horizontal Cylindrichnus concentricus (Cy) and ?Palaeophycus heberti (Ph) in mudstone-dominated heterolithic deposit, photography July 2008; D - ? Cylindrichnus isp. (arrow) in a completely bioturbated heterolithic deposit, photography September 2008; E - cluster of aff. Macaronichnus isp. (arrow) in mudstone-dominated heterolithic deposit, photography July 2008; F - bedding-oblique ?Rosselia isp. B (arrow) in mudstone-dominated heterolithic deposit, photography July 2008

Taylor et al., 2003; Tonkin, 2012). The limited lateral extent of the favourable areas may explain the patchiness and lateral discontinuity of bioturbation horizons. The punctuated distribution of individual ichnotaxa may reflect a punctuated occurrence of their producers on the seafloor (Morrisey et al., 1992; Buatois and Mŕngano, 2011).

The shortest and relatively brief colonization windows are represented by the rare bioturbation structures at sandstone bed boundaries. Such cases are most common at localities 6 and 8 , in association with large-scale cross-stratified and massive sandstone beds. The bioturbated boundaries of cross-strata sets are often rippled, which implies weak sand transport, and do not show Ophiomorpha. The latter, except for locality 11 , is rarely encountered and occurs mainly as single specimens at the deeper, least burrowed level of intensively bioturbated beds. At localities 5 and 12, the most abundant Ophiomorpha was found 1.5-2.5 m below the completely bioturbated top of sandstone overlain by mudstone and heterolithic deposits up to $60 \mathrm{~cm}$ thick. The benthic producers of Ophiomorpha there seem to have taken advantage of the short lacuna between the deposition of sand and the onset of muddy sedimentation. At locality 11, for comparison, the extensive dis- tribution of Ophiomorpha occurred in the local transient environment with low water energy and a suitably low rate of erratic sedimentation. Ophiomorpha is a deep-tier burrow formed in sandy substrate by prolonged animal activity, and therefore architecturally elaborate, constructed slowly and requiring an appropriately long colonization window. The colonization windows in the present case were apparently too short for the Ophiomorpha producers to thrive, allowing them little more than to burrow the substrate by a single operation of feeding, crawling, grazing or dwelling. For example, the migration of tidal dunes and bars is incremental and their top surfaces are relatively stable (Middleton and Southard, 1978; Dabrio and Polo, 1981; Allen, 1984), thereby potentially prone to bioturbation (Jumars and Nowell, 1984) and also offering a fair to good trace-fossil preservation. The scarcity of Ophiomorpha at these surfaces indicates the time windows for benthic colonization were generally too short. On the other hand, the scarcity of mud drapes in the dune cross-strata indicates that mud was kept perennially in suspension (cf. Longhitano and Nemec, 2005), which would imply a persistently high turbidity of water. These conditions might have disfavoured suspension-feeders and favoured deposit-feeders (Rhoads et al., 1972; Rhoads, 1973; Buatois and 

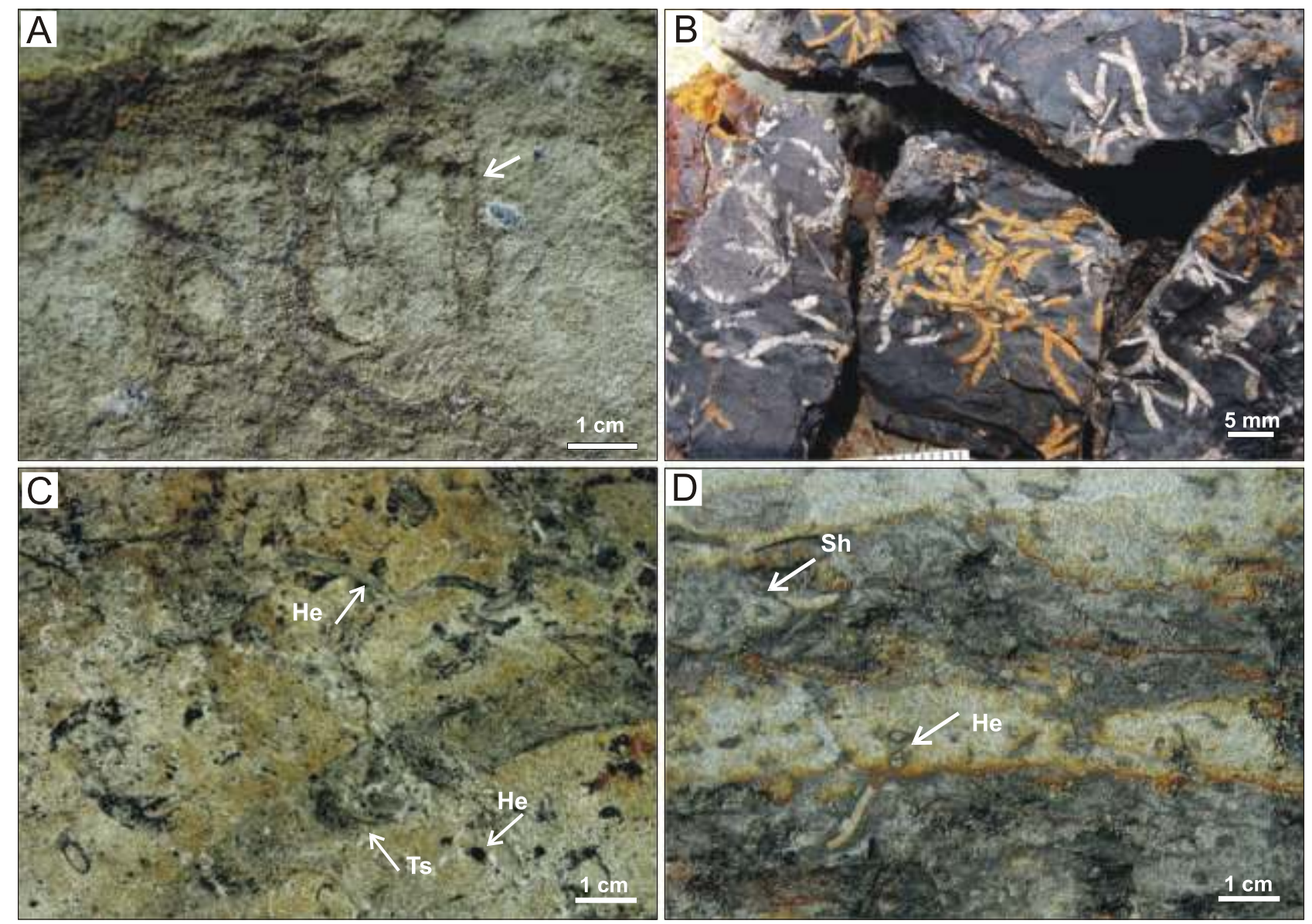

Fig. 37. Trace fossils in the Czerna Formation at locality 9 (Rakowice Małe)

A - Arenicolites sparsus (arrow) in mudstone-dominated heterolithic deposit, photography May 2017; B - bedding-plane view of Chondrites intricatus in carbonaceous mudstone, photography May 2008; C-Helminthopsis abeli (He) and cf. Thalassinoides suevicus (Ts) with faint meniscate infill on the sole of sandy siltstone, photography August 2009; D - Helminthopsis abeli (He) and ?Schaubcylindrichnus isp. (Sh) in vertical section of heterolithic deposit, photography May 2008

López-Angriman, 1992; Ranger and Pemberton, 1992; Gingras et al., 2007).

As a broader implication, the outcrop localities where the ZM deposits show least bioturbation - such as localities 1, 9 and 12 (the latter with only one richly burrowed horizon) - can be considered as representing basin areas with the highest near-bottom energy of water, probably due to tidal currents, waves and/or alongshore currents. For example, the Ophiomorpha burrows at locality 1 are rare and predominantly vertical, reflecting a substrate of little stability, although still accessible for colonisation by infauna (Howard, 1971, 1975). In contrast, outcrop localities showing extensive vertical distribution of Ophiomorpha - exemplified by locality 11 - are considered to represent areas with the lowest near-bottom energy, which may mean a relatively weak or erratic action of waves and currents.

The bioturbation structures in the ZMb deposits are generally consistent with their host lithofacies. This pertains particularly to sandstone endichnia, which are represented mainly by ophiomorphids (Seilacher, 2007), most notably Ophiomorpha nodosa, as the most pronounced elite trace fossils. This is a typical ichnotaxon of deeper-tier trace fossils in shoreface sands, especially in the middle shoreface zone (Frey et al., 1978; Pollard et al., 1993; Seilacher, 2007; Knaust and Bromley, 2012). The shallow tiers, including epichnia, consist of ichnotaxa characteristic of a wider spectrum of environments, including the whole shoreface and out to the upper offshore zone. Ichnotaxa characteristic of low bottom energy, such as Thalassinoides, are most frequent in this assemblage.

Howard $(1971,1975)$ and other authors have noted that the Ophiomorpha systems tend to shift from predominantly horizontal to vertical as the bottom energy level increases. Such a change may accompany, for example, the regressive progradation of the middle shoreface onto the lower shoreface. Accordingly, the predominance of horizontal ophiomorphs in horizons of their highest concentrations at localities 5, 11 and 12 may be considered as recording relatively low-energy conditions in these areas at the time of the bioturbation. The notion of lower shoreface conditions for horizontal Ophiomorpha burrowing seems to be true at locality 11 , whereas the low-energy conditions for such burrowing at the two other localities was due to a transient backbeach lagoonal environment (see previous section).

As a whole, the trace-fossil assemblage of the ZMb comprises ichnotaxa characteristic of the Skolithos Ichnofacies and the Cruziana Ichnofacies (Seilacher, 1963, 1967; Buatois and Mángano, 2011; Pemberton et al., 2012). The former, recognized already by Leszczyński (2010), is represented by the most numerous burrows Ophiomorpha nodosa and subordinate solitary occurrences of Diplocraterion, Rosselia and Macaronichnus. The ichnofacies flag ichnogenus Skolithos, as 

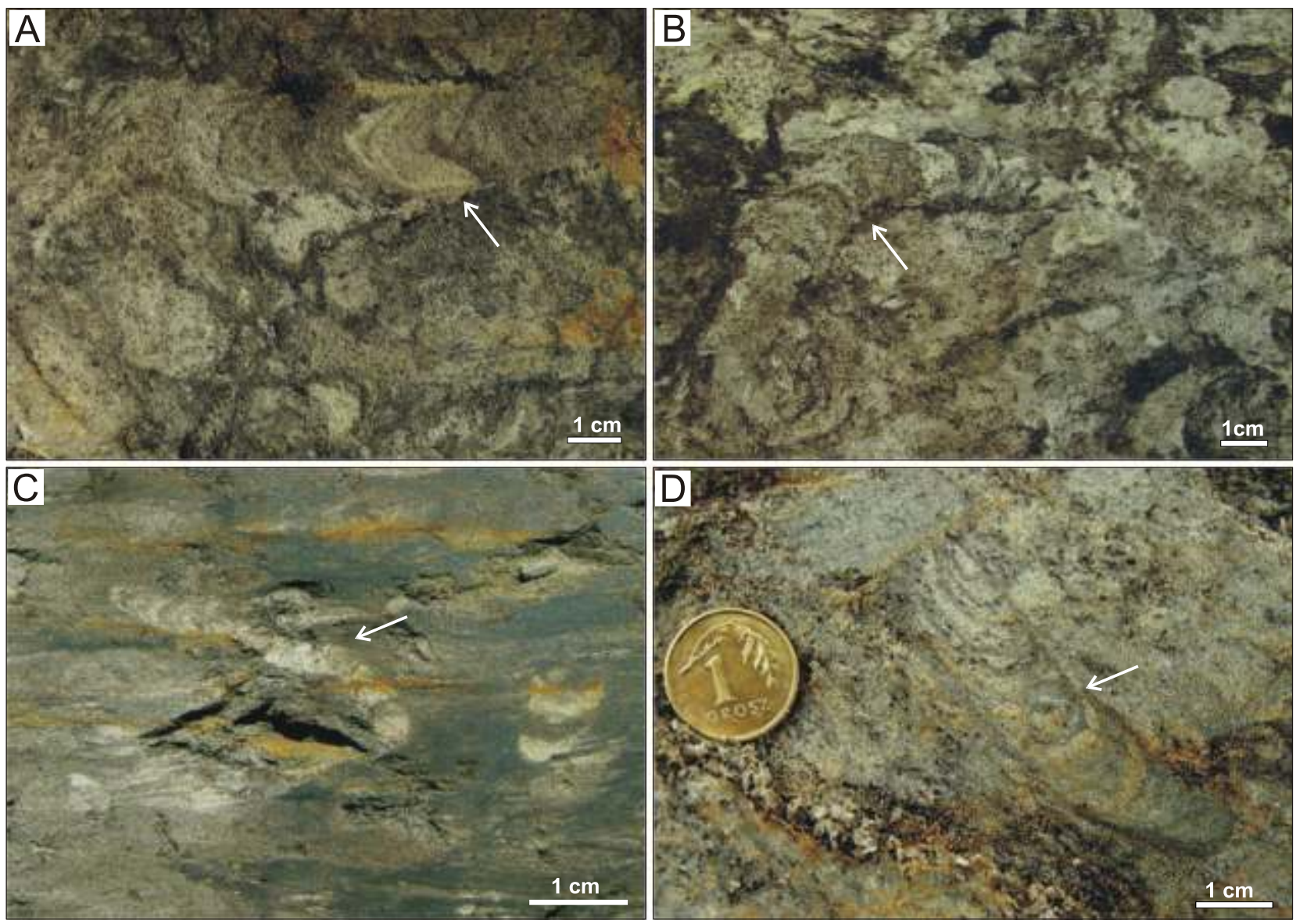

Fig. 38. Bioturbation structures in heterolithic deposits above the basal coal in the Nowogrodziec Member of the Czerna Formation at locality 9 (Rakowice Małe)

A - plan view of ? Taenidium baretti (arrow) in muddy sandstone, photography August 2008; B - plan view of ?Taenidium baretti (arrow) in muddy sandstone, photography July 2007; C - vertical section of ? Taenidium isp. A (arrow) in sandy mudstone, photography July 2008; D - vertical section of ?Taenidium isp. B (arrow) in muddy sandstone, photography August 2009

well as Arenicolites and Cylindrichnus, have not been found. This impoverished Skolithos Ichnofacies indicates an ecologically stressed environment (cf. MacEachern et al., 2007a). The nature of this stress is unclear, as it could be due to the turbidity of water, high sedimentation rate and/or paucity of nutrients.

The Skolithos Ichnofacies is characteristic of moderately to well-sorted, shifting sandy substrates with variable rates of sedimentation and erosion and with nutrient mainly in suspension (Anderson and Droser, 1998; MacEachern et al., 2012). It is typical of lower intertidal to shallow subtidal settings and thrives in moderate to high-energy conditions of the sandy foreshore, shoreface, tidal inlets and channels, sandy shoals and bars, and also sandy deep-sea fans (Pollard et al., 1993; MacEachern et al., 2012). The dominance of $O$. nodosa in the present case supports the notion of shoreface sedimentation, which is consistent with the host lithofacies. Furthermore, the distribution of $O$. nodosa suggests that the sandy substrate was mainly too mobile to be colonised by Ophiomorpha producers. Substrate instability and possibly water turbidity might also be responsible for absence of other ichnotaxa characteristic of the Skolithos Ichnofacies.

The assemblages of Thalassinoides and solitary or rare occurrences of other ichnotaxa on surfaces bounding sandstone beds are a record of the activity of epibenthos and shallow-tier endobenthos, sporadically with single Ophiomorpha burrows, and can be considered as a proximal expression of the
Cruziana Ichnofacies. Notably, Thalassinoides occurs on the same bedding surface as other ichnotaxa or closely below it. The recognition of Thalassinoides burrows depended strongly on the accessibility of bedding surfaces. Therefore, these trace fossils were found mainly in quarries with numerous sandstone blocks (localities 5, 6 and 8) and as single specimens in some others (e.g., localities 1, 4 and 9) or were not found because of the lack of accessible bedding surfaces. The occurrence of these burrows on poorly to moderately bioturbated surfaces draped with thin mud suggests episodes of low bottom-water energy. The lack of Ophiomorpha in the sandstone below could have been caused by too high water turbidity. The producers of Thalassinoides, callianassid shrimps, are known to prefer areas with a low sedimentation rate (Müller, 1970), thereby covered with fine-grained sediment. The Thallasinoides burrows often appear to have been deformed. These trace fossils, formed on mud-draped sand and made of a sandy mud, were apparently susceptible to compactional fluidization and deformation after being covered by a thick sand.

It is worth noting that the Ophiomorpha burrows in the ZMb vary with respect to the type of material forming pellets, the form and style of lining and the course pattern. This variability may be due to the involvement of several decapod taxa (Weimer and Hoyt, 1964), differing in the time span and style of substrate exploitation and in the available type of sediment for pellet production. The conical and spiky shape of pellets can be explained by 

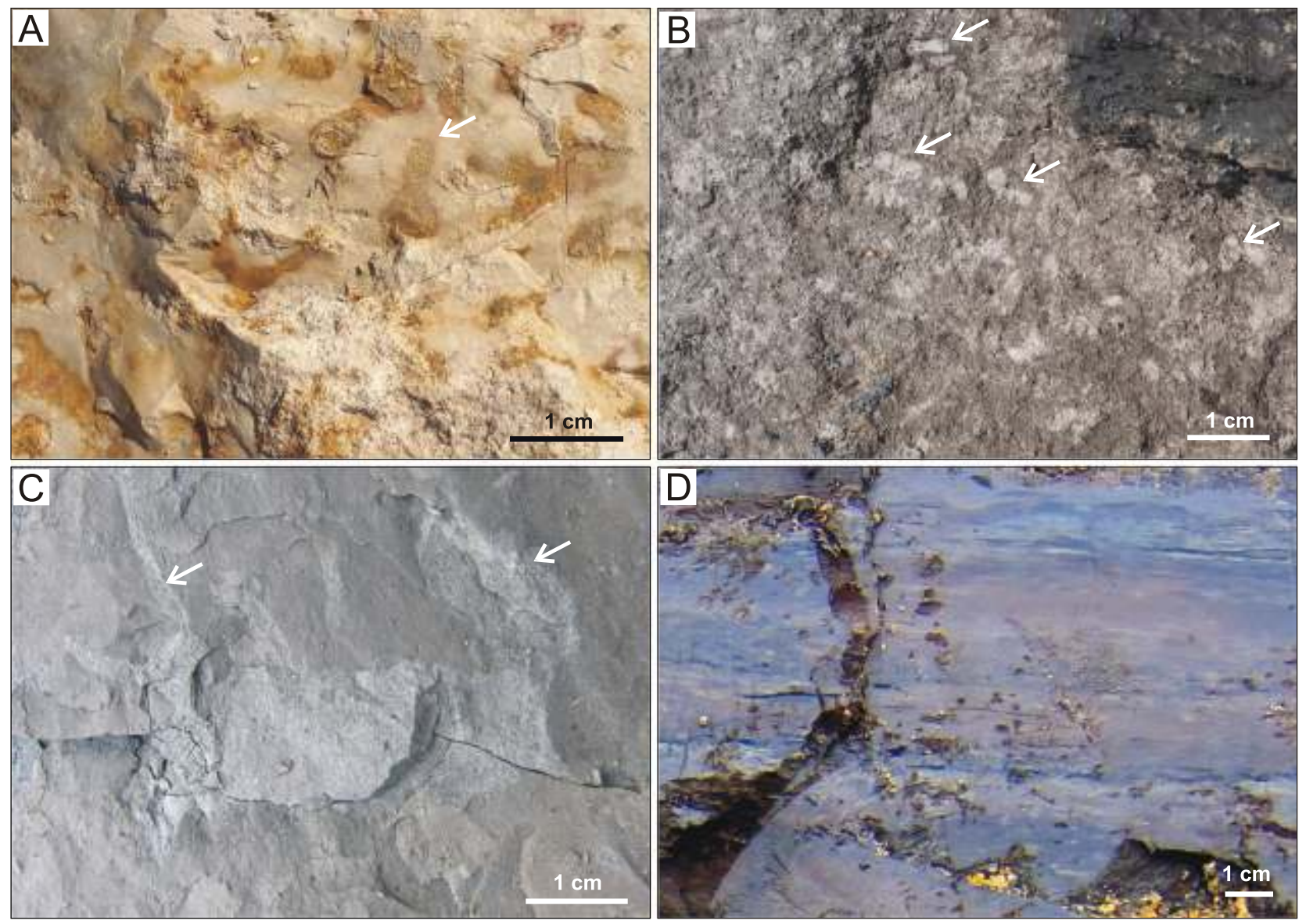

Fig. 39. Undetermined trace fossils in the lowest part of the Czerna Formation at locality 12 (Osiecznica)

A - enigmatic branched 3D swirls (arrow) in clayey mudstone; B - enigmatic 3D swirls (arrows) in muddy sandstone; C - enigmatic branched lumps (arrows) in mudstone; D - bioturbational mottling in clayey mudstone. All photographs May 2017

the mechanical compression of muddy pellets during the early diagenetic compaction of sediment (Bromley and Pedersen, 2008; Boyd et al., 2012). The irregular massive linings in some Ophiomorpha are exclusively those made of mud.

The frequently observed impoverishment of pellets in $\mathrm{Fe}$ oxide/hydroxide is considered a consequence of their original content of unstable organic matter and hence a lower Eh than in the surrounding sediment. In such conditions, the dissolved Fe would migrate out of the pellets to precipitate as a halo around the pellets or the entire burrow.

\section{LITHOFACIES AND TRACE-FOSSIL RECORD OF THE LOWER PART OF CZERNA FM.}

The lithofacies and trace fossils in the basal part of the CFm indicate a notably different and more complex depositional setting than that of the underlying ZMb. Significant differences in depositional conditions are also recorded within and between the three longest outcrop sections of the CFm (localities 5, 9 and 12). At these localities, as well as at localities 1 and 4 where only the basal part of CFm is preserved, the CFm sedimentation commenced with clayey and muddy lithofacies indicating deposition in a low-energy lagoonal or lacustrine setting. This environment, initially stressed by an elevated salinity and deficit of nutrients, might have been a shallow lagoon left behind by the late mid-Coniacian regression or a system of backshore ponds collecting rainwater drained from the emergent sandy areas. A deficit of nutrients is suggested by the lack of recognizable bioturbation structures, whereas the white, orange and red colours of the claystones and mudstones indicate well-aerated bottom conditions, unfavourable for nutrient production. Freshwater conditions prevailed and the lake margins came locally to host low vegetation, as evidenced by palaeosol at locality 5 , where also a thin lens of coal with driftwood fragments had formed.

These early-stage deposits pass upwards into greenish-grey to grey mudstones and siltstones with plant-root traces and thin coal layers, as observed at localities 5 and 9, which indicates cessation of nutrient deficit and the lake transformation into a marsh with transient peatbogs. At localities 4 and 12, the light colour early deposits are overlain by dark grey to black mudstones rich in tiny plant detritus, apparently washed from an adjacent marsh. The blackish deposits suggest poorly aerated to anoxic bottom conditions. At locality 1 , in contrast, the muddy lacustrine lithofacies are overlain by deltaic and possibly fluvial deposits, preserved there in the uppermost part of the outcrop section.

Somewhat puzzling is the palaeogeographic context of these lagoonal/lacustrine deposits at localities 4 and 12, as the latter is close to the basin's original margin, whereas the former is $1.8 \mathrm{~km}$ basinwards from locality 5 . It is possible that the ma- 
rine regression in southeastern part of the basin was leaving behind a plain with lagoon relics as shallow ponds, of which some were converted into backshore lakes faster than the others were by local rainwater drainage. The notion of a relic lagoon at locality 4 is supported by the presence of extensive, Nerinea-dominated shell lags at the top of the underlying ZMb. Likewise, the occurrence of taxonomically undetermined minute bioturbation structures in the lowest part of the CFm succession at locality 12 (Fig. 39) indicates dysaerobic, brackish pond-floor conditions. The area and depth (i.e., volume) of the backshore lagoon relics and their location with respect to rainwater drainage might render the rate of their transformation into freshwater ponds highly varied, with their salinity decline inhospitable to fauna. These conditions would explain the faint signs of mud bioturbation at some localities and their virtual lack at the others.

The subsequent sedimentation showed major differences between the individual localities. At locality 5 , the peatbog environment was drowned by the sea and another phase of sedimentation ensued in a low-energy, well-aerated and nutrient-devoid environment, as recorded by a thin mudstone package overlying the upper coal bed. Its gradual upward transition through heterolithic deposits into a unit of cross-stratified sandstone indicates encroachment of a shoal-water delta (cf. Olariu and Bhattacharya, 2006). The occurrence of Ophiomorpha nodosa in the middle part of the package and an ichnofabric reminiscent of Thalassinoides at its completely bioturbated top indicate a return of marine realm in this area. Further sedimentation, represented by deposits in the poorly accessible uppermost part of the outcrop, seems to have occurred in a delta-plain environment. The lowermost, fining-upwards sandy package still bears Ophiomorpha in the lower part and shows Thalassinoides-like ichnofabric at the completely bioturbated top, which suggests a marine-influenced and abandoned mouth of a distributary channel. The capping mud-dominated heterolithic deposits lack trace fossils and show a whitish to reddish colouration, which suggests sedimentation in a barren, low-energy environment of interdistributary alluvial floodplain.

Somewhat similar depositional conditions are recorded at locality 4, where the blackish mudstones pass upwards into a thin package of heterolithic deposits increasingly richer in sand and overlain by poorly preserved sandstones. This coarsening-upwards lithofacies succession suggests deposition as a prograding mouth bar of a shoal-water delta.

A most interesting variety of sedimentation occurred above the upper coal bed at locality 9, as initially reported by Leszczyński (2010). Bioturbation structures in the coal bed, particularly Thalassinoides and Teredolites, together with the plant-rooted seatearth below the coal and the trace-fossil assemblage in the overlying heterolithic deposits (with Asterosoma, Teichichnus, Palaeophycus, Cylindrichnus and Arenicolites), indicate marine invasion and a rapid transformation of the peatbog environment into a microtidal margin of a lagoon gradually impoverished in sand supply by further drowning. The occurrence of Teredolites clavatus at the coal-bed top indicates incursion of marine waters directly onto the peatbog surface, as this trace fossil, formed in wood, is produced by organisms not tolerating freshwater and restricted to environments with a salinity ranging from brackish to fully marine (Bromley et al., 1984; Savrda, 1991; Savrda et al., 1993). The trace-fossil suite in the coal bed represents the archetypal Teredolites Ichnofacies (see Gingras et al., 2004; MacEachern et al., 2007a; Buatois and Mágnano, 2011), of which Thalassinoides is a rare but persistent component (Gingras et al., 2002; MacEachern et al., 2007a).

Sedimentation of the overlying heterolithic deposits at locality 9 occurred in a shallow subtidal lagoon environment, as indi- cated by the fine-grained deposits, their intense bioturbation by an impoverished Cruziana Ichnofacies lacking open-marine ichnofossils such as Rhizocorallium, the absence of shelly fauna and the lack of tempestites. The complete bioturbation of the sand-dominated lower part of these deposits implies ecological conditions exceptionally favourable to benthic fauna, albeit in a stressed environment with an impoverished Cruziana Ichnofacies in its proximal expression (MacEachern et al., 2007b). The gradual upward decline of sand content, decrease of bioturbation intensity, disappearance of large burrows (such as Palaeophycus isp., Thalassinoides, Asterosoma and Teichichnus) and expansion of diminutive vermiform burrows suggest development of environmental stress due to salinity reduction and possibly a decrease of bottom-water oxygenation (cf. MacEachern et al., 2007b; Hauck et al., 2009; Leszczyński, 2010; Gingras and MacEachern, 2012). The most probable cause of this upward change might have been an increasing isolation of the area from the open sea by a regressive sand barrier. The overlying package of dark grey to greenish mudstones, lacking both recognizable trace fossils and body fossils and bearing several clay ironstone (siderite) horizons, indicates deposition in a stressed environment of low water energy, reduced salinity and oxygenation, and possibly increased turbidity (cf. MacEachern and Pemberton, 1992; MacEachern et al., 1999).

The topmost part of the succession preserved at locality 9 comprises a thin bed of coaly mudstone overlain by sandstone-dominated heterolithic deposits. The mudstone shows an upwards-increasing bioturbation intensity with a complex trace-fossil assemblage of cross-cutting ichnotaxa indicative of benthic anoxia. The subsequent increase of sand supply was probably accompanied by an increased aeration of bottom water, which caused penetration of the organic-rich mud by animals whose oxygen needs became satisfied. Chondrites was the first to appear, followed by Asterosoma and Thalassinoides. The appearance of Chondrites suggests an increase of water salinity, apparently due to an improved connection of this area with the open sea. The coaly mudstone indicates bottom water stagnation and is considered to represent maximum flooding (Leszczyński, 2010), whereas the resumption of sand supply probably reflects an early shoreface re-advance with the onset of the mid-Coniacian normal regression (Fig. 3).

In summary, the sedimentary succession at locality 9 - with its pattern of coarsening-upwards lithofacies packages, an impoverished Cruziana Ichnofacies in the lower part and a distal expression Skolithos Ichnofacies (sensu MacEachern et al., 2007a) at the top - indicates shoreface progradation punctuated by episodic rises of relative sea-level. Relatively high bioturbation intensity and rich brackish fauna (see Milewicz, 1965,1970 ) indicate deposition in a low- to high-energy environment richly supplied with nutrient, perhaps due to freshwater input. Rich plant detritus in fine-grained deposits suggest a possible interdistributary bay of a deltaic system (Leszczyński, 2010). Shell lags might have been spread by storm events. Sandstone wedges with low-angle parallel stratification may represent minor mouth bars (Elliott, 1974), which means splays emplaced sideways into the embayment by delta distributary system during major floods.

At localities 4 and 12, the blackish mudstones of ZMb pass upwards into a thin unit $(0.7 \mathrm{~m})$ of coarsening-upwards heterolithic deposits overlain by a prominent sandstone unit that is more than $2 \mathrm{~m}$ thick (top uncertain) at the former locality and $\sim 3$ $\mathrm{m}$ thick (with sharp top) at the latter locality. Thin, curved, white mud-filled vertical structures at the sandstone top at locality 12 are reminiscent of plant-root traces and suggests emergence. The coarsening-upwards succession at these localities indi- 
cates rapid shallowing due to the progradation of either a shoreface with a vegetated backbeach area or a shoal-water delta with a vegetated top plain. The small thickness of the succession and the rapid upward transition from mudstone to sandstone indicate rapid progradation, which is consistent with the notion of shoal water. This regressive succession at locality 12 is overlain by another coarsening-upwards succession of a thicker mudstone unit $(6.5 \mathrm{~m})$ overlain by heterolithic deposits $(0.5 \mathrm{~m})$ and sandstone unit $(4 \mathrm{~m})$, indicating a new cycle of drowning and progradation. The sandstone has a sharp top and is overlain by a unit of dark grey mudstones, $1.1 \mathrm{~m}$ thick, covered erosionally by Pleistocene deposits. The mudstone units are a record of marine drowning and apparently represent a rapid transformation of a transient coastal flat, locally vegetated, into an extensive shallow lagoon. No features indicative of open-marine sedimentation have been recognized. The short-term transgressive-regressive cyclothems support further the notion of a punctuated nature of the mid-Coniacian regression in the basin.

\section{PALAEOGEOGRAPHIC IMPLICATIONS}

The depositional palaeoenvironments recognized in the Coniacian succession in the southwestern part of the North Sudetic Synclinorium show great lateral variation and frequent stratigraphic changes, which points to the palaeogeographic complexity of the shallow-marine basin and its high sensitivity to sea-level fluctuations. Sedimentation of the ZMb occurred at the outlet of a strait (Fig. 40A) connecting the North Sudetic Basin with the Intrasudetic Basin and with the Bohemian Basin farther to the south-east (cf. Milewicz, 1997; Voigt et al., 2008). The strait confinement and basin elongation enhanced tidal currents, whereby the interpreted tidal sand bars formed at the basin axis in a shoreface environment that extended from both of the basin flanks. The accumulation of sediments and shoreline advance drove a normal regression, which turned into a forced regression when the rate of sea-level fall outpaced the rate of basin subsidence. The shallow strait was emerged and its sediments eroded, while the shrunken basin became surrounded by lagoonal coastal plains and local shoal-water deltas (Fig. 40B). The marine sedimentation of $\mathrm{ZMb}$ in the study area ended as the sea retreated to the north-west, the coastal plains merged - hosting stagnant freshwater ponds and marshes and an extensive erosion occurred (Fig. 40C). A result was the late mid-Coniacian hiatus (Fig. 3). The absolute depth of erosion is unknown, but the hiatus increases towards the basin margins, particularly to the south-east (Milewicz, 1997). The eroded sediment accumulated as a lowstand system in the unexposed northwestern part of the basin, where marine sedimentation continued (Fig. 40C), as indicated by boreholes N-14, N-26 (Bossowski et al., 1976; Bossowski, 1991a), N-27 (Bossowski et al., 1977), Jagodzin 1 (Marciński, 1970) and Węgliniec IG 1 (Milewicz, 1966, 1997). It is possible that the lowstand included formation of an extensive transient coastal lake or salinity-losing lagoon (Fig. 40C), as inferred from the package of greyish white and orange to reddish claystones and mudstones, several metres thick, at the top of the ZMb (Milewicz, 1965; Leszczyński, 2010). If this inference is correct, only relics of this lake/lagoon remained after erosion, whereas the corresponding coastal barrier to the north-west would now be buried in the unexposed coeval part of the basin. An occurrence of siltstone with plant-root traces (palaeosol) $5.9 \mathrm{~m}$ above the nominal top of ZMb in borhole Z-4 near the basin axis indicates that this area remained emerged for a short time even after the infilling of a hypothetical lake.

The sandstones and lacustrine pelitic deposits at the ZMb top are sharply overlain by the transgressive marine deposits of the basal CFm. The coastal plain, as an extensive flat area, was drowned rapidly by the sea expansion towards the south-east (Fig. 40D) and the transgression was insignificantly diachronous. Typical marine and brackish ichnofossils occur in the upper coal bed at locality 9 , directly above it at locality 5 and above the palaeosol in well Z-4. The basin flanks were also inevitably drowned, but little of the transgressive record there is presently preserved after the basin's tectonic inversion and transformation into a synclinorium. Barrier-sheltered lagoons formed locally on the flanks, as shown by the coeval lagoonal deposits at locality 12 . In its south-east extent, the transgression reached at least locality 5 , but it remains unclear from the CFm relics if there were any narrow embayments reaching farther in this direction. There is no evidence of marine deposits in the lower CFm deposits preserved at locality 1 .

It seems that the late Coniacian transgression had quickly established its landward limit and then proceeded until the early Santonian with little expansion of the marine area and with shoreline aggradation, rather than significant further retreat (Fig. 3; Milewicz, 1997; Walaszczyk, 2008). The transgression was followed by a normal regression punctuated by marine incursions (Fig. 3). The evidence from outcrops and boreholes indicates that the normal-regressive systems tract involved shoal-water deltas, built mainly by a fluvial system draining the emerged former strait area, while the abrupt marine invasions were forming transient interdistributary bays and shallow coastal lagoons. The higher part of the CFm, not covered by the present study, shows evidence of continued lagoonal-deltaic sedimentation followed by fluvial sedimentation in the middle to late Santonian (Milewicz, 1965, 1970, 1997, 2006; Alexandrowicz, 1976; Górniak, 1986).

The basinal palaeogeographic changes, recorded as local changes in environmental conditions, were clearly driven by small and large fluctuations of the basin bathymetry, but the exact cause of these latter is unclear because both eustatic and tectonic forcing could be involved. The basin's succession of transgressive and regressive trends is only roughly comparable to that in the neighbouring Bohemian Basin to the south-east, where some eustatic influences have been inferred (Nádaskay and Uličný, 2014). The Cenomanian-Santonian succession in the North Sudetic Basin (Fig. 3) seems to reflect broadly the eustatic changes postulated by Haq (2014: fig. 3). The midCenomanian to earliest Turonian stepwise transgression in the basin (Fig. 3) corresponds to the sea-level rise KCe3-KTu2 (Haq's notation) of a 2 nd-order eustatic cycle. The early Turonian-Coniacian regressive trend in the basin, interrupted by a late Turonian short-term transgression (Fig. 3), may be an echo of the 2 nd-order eustatic fall KTu2-KSa1 and its component 3 rd-order major fluctuations, such as the late Turonian event KTu4-5 with a bathymetric amplitude $>50 \mathrm{~m}$. The end-Coniacian transgression followed early to middle Santonian regression in the basin (Fig. 3) might only be linked to 3rd-order eustatic fluctuations (such as event KSa1-3), as the global 2nd-order trend had stabilized at that time (Haq, 2014: fig. 3). In summary, the basin's bathymetric changes might broadly reflect a combination of 2nd- and 3rd-order eustatic cycles, obscured to some extent by the fluctuations in sediment supply driven intrinsically by palaeogeographic changes and by the short-term transgressive-regressive cycles recorded as lithofacies packages $<10 \mathrm{~m}$ thick. 

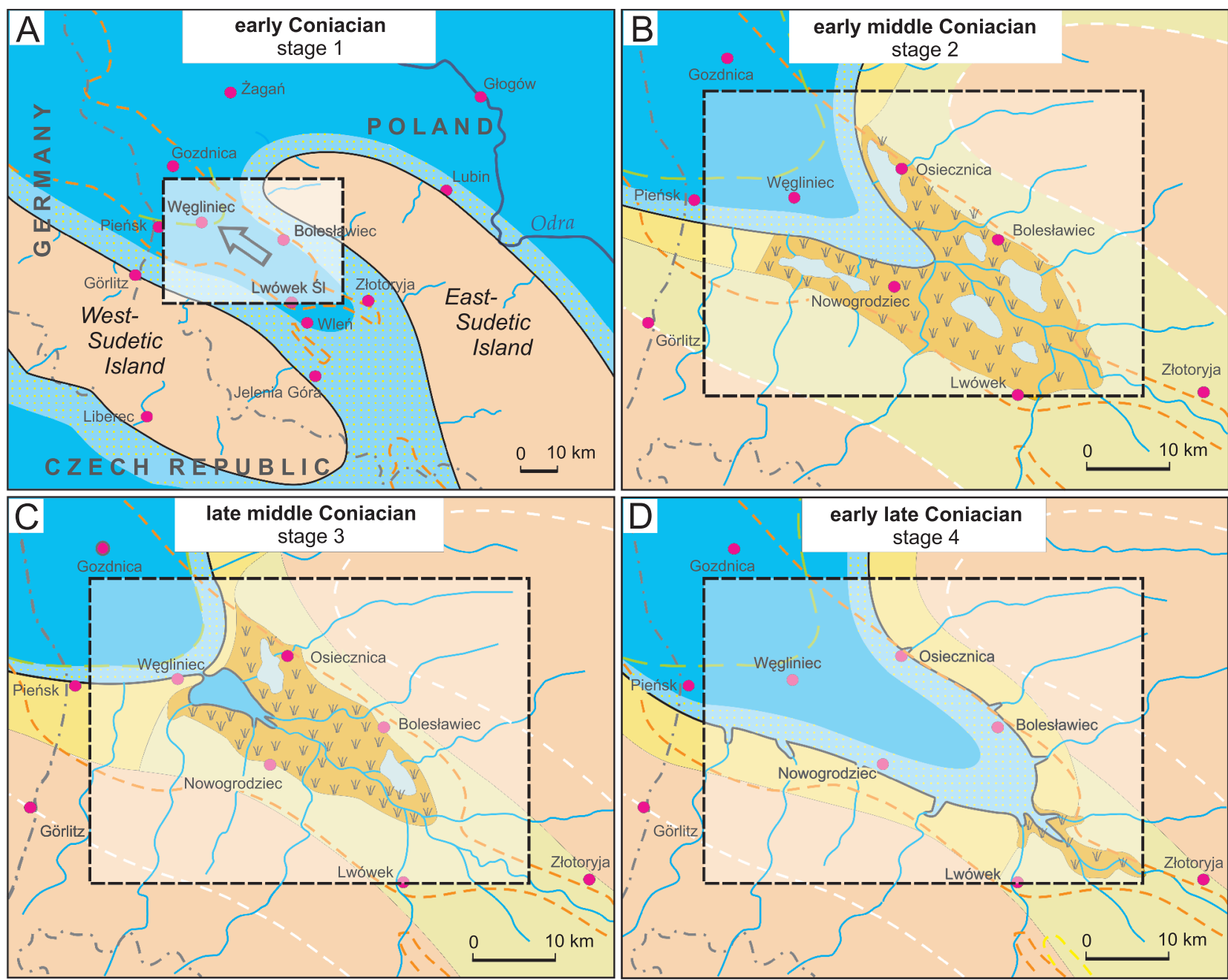

land with landscape type unspecified

(mud dominated bottom) sea shoreface (sand dominated bottom) lake

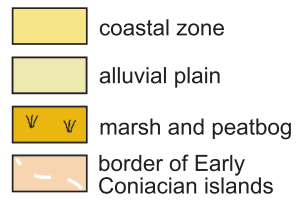

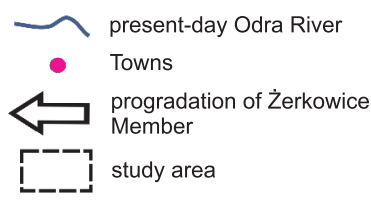

Fig. 40. Schematic interpretation of the palaeogeographic development in the study area

Hypothetical interpretation based on the present study and earlier published suggestions, with chronostratigraphy after Walaszczyk (2008). A - Stage 1 (early Conician): marine sedimentation of the Żerkowice Member, strongly influenced by tidal currents enhanced by the strait confinement; B - Stage 2 (early middle Coniacian): normal regression leads to extensive lagoonal and lacustrine/paludine sedimentation in the uppermost part of the Żerkowice Member; C - Stage 3 (late middle Coniacian): forced regression causes expansion of a stagnating terrestrial plain with relic lagoons, freshwater lakes and peat-forming mires and with extensive fluvial erosion, which results in a non-depositional/erosional hiatus in the southeastern part of the basin; D - Stage 4 (late Coniacian): rapid marine transgression occurs, non-deposition in broad paludine areas but forming local barrier-sheltered lagoons on basin flanks, causing the onset of Czerna Formation sedimentation; the transgression turned into a normal regression in the late early Santonian (Fig. 3)

On the other hand, the basin was an actively subsiding synclinal trough and was about to be tectonically inverted as a synclinorium by the Alpine orogeny, which means that a tectonic forcing may have played a significant role in intrabasinal bathymetric changes and in obscuring their eustatic signal (cf. Richardt and Wilmsen, 2013; Nádaskay and Uličný, 2014). The short-term transgressive-regressive cycles in the basin are attributed to pulses of its incremental subsidence, and it also cannot be precluded that the late Coniacian forced regression was a first sign of compressional tectonic uplift in the area separating the North Sudetic and Intrasudetic basins.

\section{CONCLUSIONS}

- Lithofacies and ichnofossils of the middle to late Coniacian deposits in the southeastern part of the North Sudetic Synclinorium indicate a wide range of shallow marine, deltaic and transient lagoonal, lacustrine and paludine palaeoenvironments, with a stratigraphic record of shoreline shifts and gradual to rapid palaeogeographic changes. Trace fossils form a high-diversity assemblage of a stressed expression of the Skolithos Ichnofacies, dominated by Ophiomorpha nodosa, and a proxi- 
mal to distal expression of the Cruziana Ichnofacies, including Thalassinoides and rare specimens of diverse other ichnotaxa. Ichnological evidence supports and supplements sedimentological palaeoenvironmental interpretations.

- Lithofacies of the mid-Coniacian erkowice Member of the Rakowice Wielkie Formation indicate deposition in a high-energy sandy shoreface environment dominated by waves, tidal currents and wave-generated alongshore currents, with a gradual encroachment of shoal-water deltas. The depositional succession is a record of a normal (progradational) regression that was leaving behind a coastal plain with relic lagoons turning into freshwater ponds and peat-forming marshes. The regression culminated in a mid-Coniacian forced regression, which caused erosion of the emerged area and resulted in a hiatus separating the erkowice Member from the overlying Czerna Formation of late Coniacian to Santonian age.

- Lithofacies of the lowest part of the Czerna Formation indicate rapid marine re-drowning of the emerged southeastern part of the basin and development of a system of lagoons. The marine transgression proceeded with shoreline aggradation and limited further retreat, before turning into another normal regression - driven by progradation of a sandy shoreface and associated shoal-water deltas. The regression was punctuated by minor marine incursions forming interdistributary bays and re-establishing transient lagoons, the latter often transformed gradually into freshwater ponds with marshes.

- Trace fossils in the erkowice Member are generally rare (BI mainly 0-2), but there are isolated and laterally discontinuous levels of high to complete bioturbation $(\mathrm{BI}=5$ to 6$)$ in the top parts of some thick sandstone beds or bed packages, particularly where draped with a mudstone layer. This pattern of bioturbation is consistent with the notion of high-energy bottom conditions and vigorous sand transport, and indicates brief local colonization windows in an environment with sparse nutrients and with mud kept perennially in suspension. Intense bioturbation occurred on the stable top surfaces and in forefront hydraulic shadows of incrementally migrating tidal bedforms such as large dunes and sand bars. The trace-fossil assemblage comprises an impoverished Skolithos Ichnofacies, represented by numerous Ophiomorpha nodosa and subordinate solitary Diplocraterion, Rosselia and Macaronichnus, and a proximal expression of the Cruziana Ichnofacies represented by Thalassinoides and solitary to rare specimens of diverse other ichnotaxa on surfaces bounding sandstone beds.

- The studied lower part of the Czerna Formation shows a much richer and more diversified assemblage of trace fossils, ranging from a few undetermined ichnotaxa in Osiecznica to 17 ichnogenera in Rakowice Małe, including plant-root traces in seatearth horizons. The occurrence of Thalassinoides and Teredolites in the coal bed above the formation base in Rakowice Małe, together with the trace fossils in the overlying heterolithic deposits (including Asterosoma, Teichichnus, Palaeophycus, Cylindrichnus and Arenicolites), is an ample evidence of a rapid marine transgression that instantly transformed the coastal-plain peatbog environment into a microtidal lagoon. The trace-fossil assemblage comprises the Teredolites Ichnofacies, a proximal expression of the Cruziana Ichnofacies and a distal expression of the Skolithos Ichnofacies, the combination of which highlights further the short-term changes of environmental conditions recorded by the lithofacies.

- The palaeogeographic changes recorded by the sedimentary succession reflect bathymetric fluctuations corresponding to a combination of 2 nd- and 3 rd-order eustatic cycles, with their signal obscured by palaeoenvironmental changes in sediment supply and by intrabasinal tectonic forcing. Pulses of incremental tectonic subsidence were likely responsible for the short-term transgressive-regressive cycles recorded as lithofacies packages $<5 \mathrm{~m}$ thick, best pronounced in the Czerna Formation.

Acknowledgements. The study was funded by a Jagiellonian University DFG research grant. For the permissions to do research in the sandstone quarries and study their documentations, the author thanks the management of the company Hofmann Natursteinwerke Polen $\mathrm{GmbH}$, particularly B. Jarema and K. Gawron (quarries at Wartowice and Skała); the company Kamieniarz in Kielce (quarries at Nowa Wieś Grodziska, Czaple and eliszów); the company Kopalnie Piaskowca in Bolesławiec (quarries at Rakowice Małe and erkowice), particularly A.M. Sroka; and the company Kopalnia i Zakład Przeróbczy Piasków Szklarskich in Osiecznica, particularly K. Kasperczyk. The author's gratitude for a free access to geological documentations is extended to the directors and trustees of the National Geological Archives in Warsaw, the Geological Archives of the Geological Enterprise "Proxima" in Wrocław and the Geological Archives of the Dolnośląskie Voievodeship in Wrocław. M. Chodowska and T. Leszczyńska are thanked for valuable field assistance. Constructive reviews by W. Nemec, D. Uličny and R. Mikulaš with editorial comments from T. Peryt, helped to improve the manuscript.

\section{REFERENCES}

Alexandrowicz, S.W., 1976. Foraminifera from the brackish Santonian deposits in the North Sudetic Basin. Rocznik Polskiego Towarzystwa Geologicznego, 46: 183-195.

Allen, J.R.L., 1982. Sedimentary structures: their character and physical Basis. Developments in Sedimentology, 30.

Altevogt, G., 1968. Erste Asterosoma-Funde in (Problem.) aus der oberen Kreide Westfalens. Neues Jahrbuch für Geologie und Paläontologie, Abhandlungen, 132: 1-8.

Anderson, B.G., Droser, M.L., 1998. Ichnofabrics and geometric configurations of Ophiomorpha within a sequence stratigraphic framework: an example from the Upper Cretaceous US western interior. Sedimentology, 45: 379-396.
Andert, H., 1934. Die Fazies in der sudetischen Kreide unter besonderer Berücksichtigung des Elbsandsteingebirges. Zeitschrift der Deutschen Geologischen Gesellschaft, 86: 617-637.

Bassyouni, A.A.F.E., El, 1984. Sedimentology of the Upper Cretaceous sandstones of the North Sudetic basin in the area between Złotoryja, Wleń, Lwówek Śląski. Ph.D. thesis, Archives of Wrocław University.

Belaústegui, Z., Gibert, J.M. de, 2013. Bow-shaped, concentrically laminated polychaete burrows: a Cylindrichnus concentricus ichnofabric from the Miocene of Tarragona, NE Spain. Palaeogeography, Palaeoclimatology, Palaeoecology, 381-382: 119-127.

Belaústegui, Z., Gibert, J.M de, Lopez-Blanco, M., Bajo, I., 2014. Recurrent constructional pattern of the crustacean burrow 
Sinusichnus sinuosus from the Paleogene and Neogene of Spain. Acta Palaeontologica Polonica, 59: 461-474.

Belaústegui, Z., Muñiz, F., Nebelsick, J.H., Domčnech, R., Martinell, J., 2017. Echinoderm ichnology: bioturbation, bioerosion and related processes. Journal of Paleontology, 91: 643-662.

Bell, C.M., 2004. Asteroid and ophiuroid trace fossils from the Lower Cretaceous of Chile. Palaeontology, 47: 51-66.

Bengtson, P., 1988. Open nomenclature. Palaeontology, 31: 223-227.

Bertling, M., Braddy, S.J., Bromley, R.G., Demathieu, G.R., Genise, J., Mikulaš, R., Nielsen, J.K., Nielsen, K.S.S., Rindsberg, A.K., Schlirf, M., Uchman, A., 2006. Names for trace fossils: a uniform approach. Lethaia, 39: 265-286.

Beyrich, E., 1849. Das Quadersandsteingebirge in Schlesien. Zeitschrift der Deutschen Geologischen Gesellschaft, 1: 390-393.

Beyrich, E., 1855. Ueber die Lagerung der Kreideformation im schlesischen Gebirge. Abhandlungen der Königlichen Akademie der Wissenschaften zu Berlin, 26: 57-80.

Blissett, D.J., Pickerill, R.K., 2004. Soft-sediment ichnotaxa from the Cenozoic White Limestone Group, Jamaica, West Indies. Scripta Geologica, 127: 341-378.

Bossowski, A. ed., 1991a. Wykroty N-14 (in Polish). Profile Głebokich Otworów Wiertniczych Państwowego Instytutu Geologicznego, 72: 1-45.

Bossowski, A. ed., 1991b. Bolesławiec N-24 (in Polish). Profile Głębokich Otworów Wiertniczych Państwowego Instytutu Geologicznego, 73: 1-64.

Bossowski, A., Bałazińska, J., Kossowska, I., Kural, K., Cieśla, E., 1978. Poszukiwanie rud miedzi w rejonie niecki północnosudeckiej (in Polish). Dokumentacja wynikowa otworów N-24 Bolesławiec i N-25 Osieczów. National Geological Archives, PIG-PIB, Warszawa, no. 2960.

Bossowski, A., Kossowska, I., Kuralowa, K., ołnierz, J., 1976. Poszukiwanie rud miedzi w rejonie niecki północnosudeckiej (in Polish). Dokumentacja wynikowa otworów N-14 - Wykroty, N-26 - Osiecznica i N-19 - Bielawa Górna. National Geological Archives, PIG-PIB, Warszawa, no. 122341.

Bossowski, A., Kossowska, I., ołnierz, J., 1977. Poszukiwanie rud miedzi w rejonie niecki północnosudeckiej (in Polish). Dokumentacja wynikowa otworów N-27 - Parowa i stref rudnych z otworów Poświętne IG-2 i Borowe IG-2. National Geological Archives, PIG-PIB, Warszawa, no. 122860.

Boyd, C., Mcllroy, D., Herringshaw, L.G., Leaman, M., 2012. The recognition of Ophiomorpha irregulaire on the basis of pellet morphology: restudy of material from the type locality. Ichnos, 19: $185-189$

Bromley, R.G., 1991. Trace fossils: biology and taphonomy. Unwin Hyman, London.

Bromley, R.G., 1996. Trace fossils: biology, taphonomy and applications. Chapman and Hall. London.

Bromley, R.G., Pedersen, G.K., 2008. Ophiomorpha irregulaire, Mesozoic trace fossil that is either well understood but rare in outcrop or poorly understood but common in core. Palaeogeography, Palaeoclimatology, Palaeoecology, 270: 295-298.

Bromley, R.G., Uchman, A., 2003. Trace fossils from the Lower and Middle Jurassic marginal marine deposits of the Sorthat Formation, Bornholm, Denmark. Bulletin of the Geological Society of Denmark, 52: 185-208.

Bromley, R.G., Pemberton, S.G., Rahmani, R.A., 1984. A Cretaceous woodground: the Teredolites ichnofacies. Journal of $\mathrm{Pa}$ leontology, 58: 488-498.

Bromley, R.G., Ekdale, A.A., Richter, B., 1999. New Taenidium (trace fossil) in the Upper Cretaceous chalk of northwestern Europe. Bulletin of the Geological Society of Denmark, 46: 47-51.

Buatois, L.A., López-Angriman, A.O., 1992. The ichnology of a submarine braided channel complex: the Whiskey Bay Formation of James Ross Island, Antarctica. Palaeogeography, Palaeoclimatology, Palaeoecology, 94: 119-140.

Buatois, L.A., Mángano, M.G., 2011. Ichnology: Organism-Substrate Interactions in Space and Time. Cambridge University Press, Cambridge, UK.
Chrząstek, A., Wypych, M., 2016. Trace fos sils from the quartz sandstones (Coniacian) from the North Sudetic Synclinorium (Czaple) (in Polish). In: XXIII Konferencja Naukowa Sekcji Paleontologicznej PTG, Abstrakty (eds. K. Pawłowska and D.Pawłowski): 27-28. 21-23.09.2016, Poznań.

Chrzastek, A., Wypych, M. 2018. Coniacian sandstones from the North Sudetic Synclinorium revisited: palaeoenvironmental and palaeogeographical reconstructions based on trace fossil analysis and associated body fossils. Geologos, 24: 29-53.

Chrząstek, A., Muszer, J,, Solecki, A., Sroka, A.M., 2018 Rosarichnoides sudeticus igen. et isp. nov. and associated fossils from the Coniacian of the North Sudetic Synclinorium (SW Poland). Geological Quarterly, 62: 181-196.

Clifton, H.E., Thompson, J.K., 1978. Macaronichnus segregatis: a feeding structure of shallow marine polychaetes. Journal of Sedimentary Petrology, 48: 1293-1302.

Coates, L., MacEachern, J.A., 2007. The ichnological signatures of river- and wave-dominated delta complexes: differentiating deltaic and non-deltaic shallow marine successions, Lower Cretaceous Viking Formation and Upper Cretaceous Dunvegan Formation, West-Central Alberta. SEPM Short Course Lecture Notes, 52: 227-254.

Cohen, K.M., Finney, S.C., Gibbard, P.L., Fan, J.-X., 2013. The ICS International Chronostratigraphic Chart. Episodes, 36: 199-204

Collinson, J.D., Mountney, N.P., Thompson, D.B., 2006. Sedimentary Structures. Terra Publishing. Harpenden.

Dabrio, C.J., Polo, M.D., 1981. Flow regime and bedforms in a ridge and runnel system, S.E. Spain. Sedimentary Geology, 28: 97-110.

Drescher, R., 1863. Ueber die Kreide-Bildungen der Gegend von Löwenberg. Zeitschrift der Deutschen Geologischen Gesselschaft, 14: 291-366.

Droser, M.L., Bottjer, D.J., 1986. A semiquantitative field classification of ichnofabric. Journal of Sedimentary Research. 56: 558-559.

Droser, M.L., Bottjer, D.J., 1989. Ichnofabric of sandstones deposited in high-energy nearshore environments: measurement and utilization. Palaios 4: 598-604.

Dyja, E., 1978. Karta otworu wiertniczego Osieczów N-25 (in Polish). National Geological Archives, PIG-PIB, Warszawa, no. 286163.

Ekdale, A.A., Harding, S.C., 2015. Cylindrichnus concentricus Toots in Howard, 1966 (trace fossil) in its type locality, Upper Cretaceous, Wyoming. Annales Societatis Geologorum Poloniae, 85: 427-432.

Elliott, T., 1974. Interdistributary bay sequences and their genesis. Sedimentology, 21: 611-622.

Engel, W., Drozdowski, S., Falecki, W., 1978. Dokumentacja geologiczna zło a rud miedzi "Wartowice" w kat. C2 (in Polish). Archives, Przedsiębiorstwo Geologiczne, Wrocław.

Fenton, C.L., Fenton, M.A., 1937. Burrows and trails from Pennsylvania rocks of Texas. The American Midland Naturalist, 18: 1079-1084.

Fillion, D., Pickerill, R.K., 1990. Ichnology of the Upper Cambrian to Lower Ordovician Bell Islands and Wabana groups of eastern Newfoundland, Canada. Palaeontographica Canadiana, 7: $1-119$.

Frey, R.W., Howard, J.D., 1975. Endobenthic adaptations of juvenile thalassinidean shrimp. Bulletin of the Geological Society of Denmark, 24: 283-297.

Frey, R.W., Howard, J.D., 1981. Conichnus and Schaubcylindrichnus: redefined trace fossils from the Upper Cretaceous of the Western Interior. Journal of Paleontology, 55: 800-804.

Frey, R.W., Howard, J.D., 1985. Trace fossils from the Panther Member, Star Point Formation (Upper Cretaceous), Coal Creek Canyon, Utah. Journal of Paleontology, 59: 370-404.

Frey, R.W., Howard, J.D., 1990. Trace fossils and depositional sequences in a clastic shelf setting, Upper Cretaceous of Utah. Journal of Paleontology, 64: 803-820.

Frey, R.W., Howard, J.D., Pryor, W.A., 1978. Ophiomorpha: its morphologic, taxonomic, and environmental significance. 
Palaeogeography, Palaeoclimatology, Palaeoecology, 23 199-223.

Frey, R.W., Pemberton, S.G., Fagerstrom, J.A., 1984. Morphological, ethological, and environmental significance of the ichnogenera Scoyenia and Ancorichnus. Journal of Paleontology, $\mathbf{5 8}$ 511-528.

Fritsch, A., 1908. Problematica silurica. In: Systeme Silurien du centre de la Boheme (ed. J. Barrande): 1-28. Barrande Fonds, Prague.

Fu, S., 1991. Funktion, Verhalten und Einteilung fucoider und lophoctenoider Lebensspuren. Courier Forschungsinstitut Senckenberg, 135: 1-79.

Fürsich, F.T., 1973. A revision of the trace fossils Spongeliomorpha, Ophiomorpha and Thalassinoides. Neues Jahrbuch für Geologie und Paläontologie, Monatshefte, (12): 719-735.

Fürsich, F.T., 1974. On Diplocraterion Torell 1870 and the significance of morphological features in vertical, spreiten-bearing, U-shaped trace fossils. Journal of Paleontology, 48: 952-962.

Gani, M.R., Bhattacharya, J.P., MacEachern, J.A., 2007. Using ichnology to determine relative influence of waves, storms, tides, and rivers in deltaic deposits: Examples from Cretaceous Western Interior Seaway, U.S.A. SEPM Short Course Notes, 52 209-227.

Gibert, J.M. de, 1996. A new decapod burrow system from the NW Mediterranean Pliocene. Revista Espańola de Paleontología, 11: 251-254.

Gibert, J.M. de, Robles, J.M., 2005. Firmground ichnofacies recording high-frequency marine flooding events (Langhian transgression, Vallés-Penedés Basin, Spain). Geologica Acta, 3 : 295-305.

Gingras, M.K., MacEachern, J.A., 2012. Tidal ichnology of shallow-water clastic settings. In: Principles of Tidal Sedimentology (eds. R.A. Davis, Jr. and R.W. Dalrymple): 57-77. Springer-Verlag, Dordrecht.

Gingras, M.K., Räsänen, M.E., Pemberton, S.G., Romero, L.P., 2002. Ichnology and sedimentology reveal depositional characteristics of shoreface parasequences in Miocene Amazonian foreland basin. Journal of Sedimentary Research, 72: 871-883.

Gingras, M.K., MacEachern, J.A., Pickerill, R.K., 2004. Modern perspectives on the Teredolites Ichnofacies: observations from Willapa Bay, Washington. Palaios, 19: 79-88.

Gingras, M.K., Bann, K.L., MacEachern, J.A., Waldron, J., Pemberton, S.G., 2007. A conceptual framework for the application of trace fossils. SEPM Short Course Notes, 52: 1-26.

Górniak, K., 1986. O sedymentacji santonu niecki północnosudeckiej i mo liwości występowania złó surowców ilastych (in Polish with English summary). Archiwum Mineralogiczne, 41: 123-134.

Haq, B.U., 2014. Cretaceous eustasy revisited. Global and Planetary Change, 114: 44-58.

Harms, J.C., Southard, J.B., Spearing, D.R., Walker, R.G., 1975 Depositional environments as interpreted from primary sedimentary structures and stratification sequences. SEPM Short Course Lecture Notes, 2

Harms, J.C., Southard, J.B., Walker, R.G., 1982. Structures and sequences in clastic rocks. SEPM Short Course Lecture Notes, 9.

Hauck, T.E., Dashtgard, S.E., Pemberton, S.G., Gingras, M.K., 2009. Brackish-water ichnological trends in a microtidal barrier-island embayment system, Kouchibouguac National Park New Brunswick, Canada. Palaios, 24: 478-496.

Häntzschel, W., 1975. Trace fossils and problematica. In: Treatise on Invertebrate Paleontology, Second edition, Part W (ed. C. Teichert): W85-W86. Geological Society of America and Kansas University Press. Lawrence, Kansas.

Howard, J.D., 1971. Trace fossils as paleoecological tools. In: Recent Advances in Paleoecology and Ichnology. Short Course Lecture Notes (eds. J.D. Howard, J.W. Valentine and J.E. Warme): 184-212. American Geological Institute.

Howard, J.D., 1975. The sedimentological significance of trace fossils. In: The Study of Trace Fossils: a Synthesis of Principles, Problems and Procedures in Ichnology (ed. R.W. Frey): 131-146. Springer, New York.
Howard, J.D., Frey, R.W., 1984. Characteristic trace fossils in nearshore to offshore sequences, Upper Cretaceous of east-central Utah. Canadian Journal of Earth Sciences, 21: 200-219.

IIgar, A., Nemec, W., 2005. Early Miocene lacustrine deposits and sequence stratigraphy of the Ermenek Basin, Central Taurides, Turkey. Sedimentary Geology, 173: 233-275.

Jumars, P.A., Nowell, A.R.M., 1984. Fluid and sediment dynamic effects on marine benthic community structure. American Zoologist, 24: 45-55.

Keighley, D.G., Pickerill, R.K., 1994. The ichnogenus Beaconites and its distinction from Ancorichnus and Taenidium. Palaeontology, 37: 305-337.

Kelly, S.R.A., Bromley, R.G., 1984. Ichnological nomenclature of clavate borings. Palaeontology, 27: 793-807.

Knaust, D., 2017. Atlas of Trace Fossils in Well Core: Appearance, Taxonomy and Interpretation. Springer International Publishing, Berlin, New York.

Knaust, D., Bromley, R.G. eds., 2012. Trace Fossils as indicators of sedimentary environments. Developments in Sedimentology, 64.

Knox, L.W., Miller, M.F., 1985. Environmental control of trace fossil morphology. Society of Economic Paleontologists and Mineralogists, 35: 167-176.

Kochanowska, J., 1988. Karta otworu wiertniczego Zebrzydowa-4 (in Polish). Archives of Przedsiębiorstwo Geologiczne we Wrocławiu PROXIMA S.A., Report No. DV/2827.

Komar, P.D., Miller, M.C., 1975. The initiation of oscillatory ripple marks and the development of plane-bed at high shear stresses under waves. Journal of Sedimentary Petrology, 45: 697-703.

Krutzsch, W., 1966. Die sporenstratigraphische Gliederung der Oberkreide im nordlichen Mitteleuropa. Abhandlungen des Zentralen Geologischen Institutes, 8: 79-111.

Ksią kiewicz, M., 1977. Trace fossils in the Flysch of the Polish Carpathians. Palaeontologia Polonica, 36: 1-208.

Leszczyński, S., 2010. Coniacian-?Santonian paralic sedimentation in the Rakowice Małe area of the North Sudetic Basin, SW Poland: sedimentary facies, ichnological record and palaeogeographical reconstruction of an evolving marine embayment. Annales Societatis Geologorum Poloniae, 80: 1-24.

Leszczyński, S., Nemec, W., 2015. Dynamic stratigraphy of composite peripheral unconformity in a foredeep basin. Sedimentology, 62: 645-680.

Leymerie, M.A., 1842. Suite du mémoire sur le terrain Crétacé du departement de L'Aube. Bulletin de la Societe geologique de France, Memoire, 5: 1-34.

Longhitano, S.G., Nemec, W., 2005. Statistical analysis of bed thickness variation in a Tortonian succession of biocalcarenitic tidal dunes, Amantea Basin, Calabria, southern Italy. Sedimentary Geology, 179: 195-224.

MacEachern, J.A., Pemberton, S.G., 1992. Ichnological aspects of Cretaceous shoreface successions and shoreface variability in the Western Interior Seaway of North America. SEPM Core Workshop, 17: 57-84.

MacEachern, J.A., Zaitlin, B.A., Pemberton, S.G., 1999. A sharp-based sandstone of the Viking Formation, Joffre Field, Alberta, Canada: criteria for recognition of transgressively incised shoreface complex. Journal of Sedimentary Research, 69: 876-892.

MacEachern, J.A., Bann, K.L., Pemberton, S.G., Gingras, M.K., 2007a. The ichnofacies paradigm: high resolution paleoenvironmental interpretation of the rock record. SEPM Short Course Notes, 52: 27-64

MacEachern, J.A., Pemberton, S.G., Bann, K.L., Gingras, M.K., 2007b. Departures from the archetypal ichnofacies: effective recognition of environmental stress in the rock record. SEPM Short Course Notes, 52: 65-93.

MacEachern, J.A., Bann, K.L., Gingras, M.K., Zonneveld, J.-P., Dashtgard, S.E., Pemberton, S.G., 2012. The ichnofacies paradigm. Developments in Sedimentology, 64: 103-138.

Maples, Ch.G., West, R.R., 1989. Lockeia not Pelecypodichnus. Journal of Paleontology, 63: 694-696. 
Marcinowski, R., Wierzbowski, A., 1975. On the nature of decapod burrows "Spongia sudolica" of Zareczny (1878). Acta Geologica Polonica, 25: 399-405.

Marciński, J., 1970. Karta otworu wiertniczego Jagodzin 1 (in Polish). National Geological Archives, PIG-PIB, Warszawa, no. 290731.

Martin, A.J., Rindsberg, A.K., 2007. Arthropod tracemakers of Nereites? Neoichological observations of juvenile limulids and their paleoichnological applications. In: Trace Fossils: Concepts, Problems, Prospects (ed. W.M. Miller, III): 478-491. Elsevier, Amsterdam.

Mángano, M.G., Buatois, L.A., 2004. Reconstructing Early Phanerozoic intertidal ecosystems: ichnology of the Cambrian Campanario Formation in northwest Argentina. Fossils and Strata, 51: 17-38.

Mcllroy, D. ed., 2004. The application of Ichnology to palaeoenvironmental and stratigraphic analysis. Geological Society Special Publications, 228: 1-493.

Messina, C., Nemec, W., Martinius, A.W., Elfenbein, C., 2014. The Garn Formation (Bajocian-Bathonian) in the Kristin Field, Halten Terrace: its origin, facies architecture and primary heterogeneity model. IAS Special Publication, 47: 513-550.

Middleton, G.V., Southard, J.B., 1978. Mechanics of sediment movement. SEPM Short Course 3 Lecture Notes, 3.

Milewicz, J., 1956. Zaburzenie utworów kredowych w Rakowicach Małych (in Polish). Przegląd Geologiczny, 4: 361-364.

Milewicz, J., 1958. Podział stratygraficzny osadów kredowych w niecce północno-sudeckiej (in Polish). Przegląd Geologiczny, 6: 386-388.

Milewicz, J., 1965. Facies of Upper Cretaceous in the eastern part of the North Sudetic Basin (in Polish with English summary). Biuletyn Instytutu Geologicznego, 170: 15-80.

Milewicz, J., 1966. Kreda z głębokiego otworu Węgliniec IG1 (in Polish). Kwartalnik Geologiczny, 10 (4) 1144-1146.

Milewicz, J., 1970. The Cretaceous of the Jerzmanice Graben (Sudetes) (in Polish with English summary). Biuletyn Instytutu Geologicznego, 239: 37-66.

Milewicz, J., 1973. Niecka północnosudecka (in Polish). In: Budowa Geologiczna Polski, tom 1 - Stratygrafia, część 2 - Mezozoik (ed. S. Sokołowski): 619-628. Wyd. Geol., Warszawa.

Milewicz, J., 1979. Distribution of Cretaceous rocks in the North Sudetic Basin (in Polish with English summary). Kwartalnik Geologiczny, 23 (4): 819-825.

Milewicz, J., 1985. A proposal of formal stratigraphic subdivision of the infill of the North Sudetic Depression (in Polish with English summary). Przegląd Geologiczny, 33: 385-389.

Milewicz, J., 1991a. On the sedimentation conditions of the Coniacian sandstones in the North-Sudetic Basin (in Polish with English summary). Acta Universitatis Vratislaviensis No 1276 , Prace Geologiczno-Mineralogiczne, 19: 47-61.

Milewicz, J., 1991b. On development of the North Sudetic Cretaceous Basin (in Polish with English summary). Biuletyn Państwowego Instytutu Geologicznego, 367: 135-141.

Milewicz, J., 1997. Upper Cretaceous of the North Sudetic Depression (litho- and biostratigraphy, paleogeography, tectonics and remarks on raw materials) (in Polish with English summary). Prace Geologiczno-Mineralogiczne, 61: 5-54.

Milewicz, J., 1998. Distribution of Coniacian sandstones in the North Sudetic Basin (in Polish with English summary). Acta Universitatis Vratislaviensis No 2004, Prace Uniwersytetu Wrocławskiego, 64: 101-109.

Milewicz, J., 2006. On Santonian deposits within the North Sudetic Basin (in Polish). Przegląd Geologiczny, 54: 693-694.

Milewicz, J., Podemski, M., Witwicka, E., 1968. New data on Upper Cretaceous in the western part of the North Sudetic Trough (in Polish with English summary). Kwartalnik Geologiczny, 12 (1): 143-152.

Miller, M.F., Curran, H.A., Martino, R.L., 1998. Ophiamorpha nodosa in estuarine sands of the Lower Miocene Calvert Formation at the Pollack Farm Site, Delaware. Delaware Geological Survey, Special Publication, 21: 41-46.
Miller, W., III, Aalto, K.R., 2008. Rosselia ichnofabric in the Miocene Pullen Formation, northwestern California: implications for the interpretation of regional tectonics. Palaios, 23: 329-335.

Mitchell, A.J., Uličný, D., Hampson, G.J., Allison, P.A., Gorman, G.J., Piggot, M.D., Wells, M.R., Pain, C.C., 2010. Modelling tidal current-induced bed shear stress and palaeocirculation in an epicontinental seaway: the Bohemian Cretaceous Basin, Central Europe. Sedimentology, 57: 359-388.

Morrisey, D.J., Howitt, L., Underwood, A.J., Stark, J.S., 1992. Spatial variation in soft-sediment benthos. Marine Ecology Progress Series, 81: 197-204.

Musstow, R., 1968. Beitrag zur Stratigraphie und Paläogeographie der Oberkreide und des Albs in Ostbrandenburg und der östlichen Niederlausitz. Geologie, 17: 1-71.

Müller, A.H., 1970. Über Ichnia vom Typ Ophiomorpha und Thalassinoides (Vestigia invertebratorum, Crustacea). Monatshefte der deutschen Akademie der Wissenschaften zu Berlin, 12: 775-787.

Nádaskay, R., Uličný, D., 2014. Genetic stratigraphy of Coniacian deltaic deposits of the northwestern part of the Bohemian Cretaceous Basin. Zeitschrift der Deutschen Gesellschaft für Geowissenschaften, 165: 547-575.

Nara, M., 2006. Reappraisal of Schaubcylindrichnus: a probable dwelling/feeding structure of a solitary funnel feeder. Palaeogeography Palaeoclimatology Palaeoecology, 240: 439-452.

Nara, M., Seike, K., 2004. Macaronichnus segregatis - like traces found in the modern foreshore sediments of the Kujukuri-hama coast, Japan (in Japanese with English summary). Journal of the Geological Society of Japan, 110: 545-551.

Olariu, C., Bhattacharya, J.P., 2006. Terminal distributary channels and delta front architecture of river-dominated delta systems. Journal of Sedimentary Research, 76: 212-233.

Partsch, J., 1896. Schlesien. Eine Landeskunde für das deutsche Volk. 1. Teil: Das ganze Land. Ferdinand Hirt, Breslau.

Pedersen, G.K., Bromley, R.G., 2006. Ophiomorpha irregulaire, rare trace fossil in shallow marine sandstones, Cretaceous Atane Formation, West Greenland. Cretaceous Research, 27: 964-972.

Pemberton, S.G., Frey, R.W., 1982. Trace fossil nomenclature and the Planolites-Palaeophycus dilemma. Journal of Paleontology, 56: 843-881.

Pemberton, S.G., MacEachern, J.A., Dashtgard, S.E., Bann, K.L., Gingras, M.K., Zonneveld, J.-P., 2012. Shorefaces. Developments in Sedimentology, 64: 563-604.

Pollard, J.E., Goldring, R., Buck, S.G., 1993. Ichnofabrics containing Ophiomorpha: significance in shallow-water facies interpretation. Journal of the Geological Society, 150: 149-164.

Po aryski, W., Brochwicz-Lewiński, W., Brodowicz, Z., Jaskowiak-Szoenejch, M., Milewicz, J., Sawicki, L., Uberna T., 1979. Geological map of Poland and adjoining countries, without Cenozoic formations (without Quaternary in the Carpathians). Wyd. Geol., Warszawa.

Ranger, M.J., Pemberton, S.G., 1992. The sedimentology and ichnology of estuarine point bars in the McMurray Formation of the Athabasca Oil Sands deposit, north-eastern Alberta, Canada. SEPM Core Workshop, 17: 401-421.

Reineck, H.-E., Singh, I.B., 1980. Depositional Sedimentary Environments. Springer, Berlin.

Retallack, G.J., 1988. Field recognition of paleosols. GSA Special Papers, 216: 1-20.

Rhoads, D.C., 1973. The influence of deposit-feeding benthos on water turbidity and nutrient recycling. American Journal of Science, 273: 1-22.

Rhoads, D.C., Speden, I.G., Waage, K.M., 1972. Trophic group analysis of Upper Cretaceous (Maestrichtian) bivalve assemblages from South Dakota. AAPG Bulletin, 56: 1100-1113.

Richardt, N., Wilmsen, M., 2013. Sequence stratigraphy of the lower Upper Cretaceous Elbtal Group (Saxony, Germany): new data from Middle Cenomanian-Upper Turonian outcrops and boreholes. Geophysical Research, EGU General Assembly 2013, Abstracts. 
Rindsberg, A.K., Kopaska-Merkel, D.C., 2005. Treptichnus and Arenicolites from the Steven C. Minkin Paleozoic footprint site (Langsettian, Alabama, USA). Alabama Paleontological Society Monograph, 1: 121-141.

Savrda, C.E., 1991. Teredolites, wood substrates and sea-level dynamics. Geology, 19: 905-908.

Savrda, C.E., Smith, M.W., 1996. Behavioural implications of branching and tube-lining in Teredolites. Ichnos, 4: 191-198.

Savrda, C.E., Ozalas, K., Demko, T.H., Hutchinson, R.A., Scheiwe, T.D., 1993. Log grounds and the ichnofossil Teredolites in transgressive deposits of the Clayton Formation (Lower Paleocene), western Alabama. Palaios, 8: 311-324.

Scupin, H., 1910. Über sudetische prätertiäre junge Krustenbewegungen und die Verteilung von Wasser und Land zur Kreidezeit in der Umgebung der Sudeten und des Erzgebirges. Zeitschrift für Naturwissenschaften, 82: 321-344.

Scupin, H., 1912-1913. Die Löwenberger Kreide und ihre Fauna. Palaeontographica, Supplement VI. Stuttgart.

Scupin, H., 1936. Zur Palaeographie des sudetischen Kreidemeeres. Zeitschrift der Deutschen Geologischen Gesellschaft, 88: 309-325.

Schlirf, M., 2000. Upper Jurassic trace fossils from the Boulonnais (northern France). Geologica et Palaeontologica, 34: 145-213.

Schlirf, M., 2012. Helicophycus seilacheri n. isp. and Biformites insolitus Linck, 1949 (trace fossils) from the Late Triassic of the Germanic Basin: their taxonomy and palaeoecological rele vance. Neues Jahrbuch für Geologie und Paläontologie, 263 $185-198$.

Seike, K., 2007. Palaeoenvironmental and palaeogeographical implications of modern Macaronichnus segregatis-like traces in foreshore sediments on the Pacific coast of central Japan. Palaeogeography, Palaeoclimatology, Palaeoecology, 252: 497-502.

Seike, K., Yanagishima, S., Nara, M., Sasaki, T., 2011. Large Macaronichnus in modern shoreface sediments: identification of the producer, the mode of formation, and paleoenvironmental implications. Palaeogeography, Palaeoclimatology, Palaeoecology, 311: 224-229.

Seilacher, A., 1963. Lebensspurren und Salinitatsfazies. Fortschritte in der Geologie von Rheinland und Westfalens, 10: 81-94.

Seilacher, A., 1967. Bathymetry of trace fossils. Marine Geology, 5: 413-428.

Seilacher, A., 2007. Trace Fossil Analysis. Springer, Berlin.

Stepanek, J., Geyer, G., 1989. Spurenfossilien aus dem Kulm (Unterkarbon) des Frankenwaldes. Beringeria, 1: 1-55.

Šimo, V., Olšavský, M., 2007. Diplocraterion parallelum Torell, 1870, and other trace fossils from the Lower Triassic succession of the Drienok Nappe in the Western Carpathians, Slovakia. Bulletin of Geosciences, 82: 165-173.

Taylor, A.M., Goldring, R., 1993. Description and analysis of bioturbation and ichnofabric. Journal of the Geological Society, 150: $141-148$.

Taylor, A.M., Goldring, R., Gowland, S., 2003. Analysis and application of ichnofabrics. Earth-Science Reviews, 60: 227-259.

Tonkin, N., 2012. Deltas. Developments in Sedimentology, 64: 507-528.

Uchman, A., 1995. Taxonomy and palaeoecology of flysch trace fossils: the Marnoso-arenacea Formation and associates facies (Miocene, Northern Apennines, Italy). Beringeria, 15: 1-114.

Uchman, A., 1998. Taxonomy and ethology of flysch trace fossils: a revision of the Marian Ksia kiewicz collection and studies of complementary material. Annales Societatis Geologorum Poloniae, 68: 105-218.

Uchman, A., 2009. The Ophiomorpha rudis ichnosubfacies of the Nereites ichnofacies: characteristics and constraints. Palaeogeography, Palaeoclimatology, Palaeoecology, 276: 107-119.

Voigt, S., Wagreich, M., Surlyk, F., Walaszczyk, I., Uličny, D., Cech, S., Voigt, T., Wiese, F., Wilmsen, M., Niebuhr, B., Reich, M., Funk, H., Michalik, J., Jagt, J.W.M., Felder, P.J., Schulp, A.S., 2008. Cretaceous. In: The Geology of Central Europe, 2 (Mesozoic and Cenozoic) (ed. T. McCann): 923-998. Geological Society, London.

Walaszczyk, I., 2008. North Sudetic Basin (Outer Sudetic Cretaceous). In: The Geology of Central Europe, 2 (Mesozoic and Cenozoic) (ed. T. McCann): 959-960. Geological Society, London.

Weimer, R.J., Hoyt, J.H., 1964. Burrows of Callianassa major Say, geologic indicators of littoral and shallow neritic environments. Journal of Paleontology, 38: 761-767.

Wetzel, A., 2002. Modern Nereites in the South China Sea - ecological association with redox conditions in the sediment. Palaios, 17: 507-515.

Williger, G., 1882. Die Löwenberger Kreidemulde, mit besonderer Berücksichtigung ihrer Fortsetzung in der preussischen Ober-Lausitz. Jahrbuch der Königlichen Preussischen Geologischen Landesanstalt, 1882: 55-125.

Zaręczny, S., 1878. O średnich warstwach kredowych w krakowskim okręgu (in Polish). Sprawozdania Komisji Fizjograficznej, Akademii Umiejętności w Krakowie, 12: 7-46.

Ziegler, P.A., 1990. Geological Atlas of Western and Central Europe. Shell Publication, Elsevier, Amsterdam.

elaźniewicz, A., Aleksandrowski, P., Buła, Z., Karnkowski, P.H., Konon, A., Oszczypko, N., Ślączka, A., aba, J., ytko, K., 2011. Regionalizacja Geologiczna Polski (in Polish). Komitet Nauk Geologicznych Polskiej Akademii Nauk, Wrocław. 